\title{
EGFRvIII : molecular insights and therapeutic potential
}

Citation for published version (APA):

Jutten, B. (2014). EGFRvIII : molecular insights and therapeutic potential. [Doctoral Thesis, Maastricht University]. Maastricht University. https://doi.org/10.26481/dis.20140613bj

Document status and date:

Published: 01/01/2014

DOI:

10.26481/dis.20140613bj

Document Version:

Publisher's PDF, also known as Version of record

\section{Please check the document version of this publication:}

- A submitted manuscript is the version of the article upon submission and before peer-review. There can be important differences between the submitted version and the official published version of record.

People interested in the research are advised to contact the author for the final version of the publication, or visit the DOI to the publisher's website.

- The final author version and the galley proof are versions of the publication after peer review.

- The final published version features the final layout of the paper including the volume, issue and page numbers.

Link to publication

\footnotetext{
General rights rights.

- You may freely distribute the URL identifying the publication in the public portal. please follow below link for the End User Agreement:

www.umlib.nl/taverne-license

Take down policy

If you believe that this document breaches copyright please contact us at:

repository@maastrichtuniversity.nl

providing details and we will investigate your claim.
}

Copyright and moral rights for the publications made accessible in the public portal are retained by the authors and/or other copyright owners and it is a condition of accessing publications that users recognise and abide by the legal requirements associated with these

- Users may download and print one copy of any publication from the public portal for the purpose of private study or research.

- You may not further distribute the material or use it for any profit-making activity or commercial gain

If the publication is distributed under the terms of Article $25 \mathrm{fa}$ of the Dutch Copyright Act, indicated by the "Taverne" license above, 


\section{EGFRvill:}

molecular insights and therapeutic potential 
C C Copyright Barry Jutten, Maastricht 2014

ISBN 978-90-9028258-9

Production Drukkerij Castro, Riemst 


\section{EGFRvIII: \\ molecular insights and therapeutic potential}

\section{PROEFSCHRIFT}

Ter verkrijging van de graad van doctor aan de Universiteit Maastricht, op gezag van de Rector Magnificus, Prof.dr. L.L.G. Soete, volgens het besluit van het College van Decanen, in het openbaar te verdedigen op vrijdag 13 juni 2014 om 12.00

$$
\text { door }
$$

\section{Barry Jutten}

geboren te Stein op 21 september 1973 


\section{Promotor:}

Prof. dr. Ph. Lambin

\section{Copromotores:}

Dr. K.M.A. Rouschop

Dr. J. Theys

Prof. dr. G. Lammering (Mediclin Robert Janker Klinik, Bonn, Duitsland)

\section{Beoordelingscommissie:}

Prof. dr. F.C.S. Ramaekers (Voorzitter)

Prof. dr. A.P. de Bruïne

Prof. dr. H.P. Rodemann (University of Tübingen, Duitsland)

Dr. J. Bussink (Radboudumc, Nijmegen)

Dr. B. Kusters (Radboudumc, Nijmegen) 


\section{Contents}

$\begin{array}{ll}\text { Outline of the thesis } & 7\end{array}$

Chapter $1 \quad$ General Introduction 11

Chapter $2 \quad$ Binding of cetuximab to the EGFRvIll deletion 37 mutant and its biological consequences in malignant glioma cells

Chapter 3 Discriminative in vivo imaging of wtEGFR and

EGFRvIII using nanobody technology

Chapter 4 The deletion mutant EGFRvIII significantly contributes to stress resistance typical for the tumour microenvironment

Chapter 5 Autophagy inhibition abrogates the survival advantages of EGFRvIII+ cells, sensitizes tumors to irradiation and improves prognosis of GBM patients

Chapter $6 \quad$ EGFR overexpressing cells and tumors are dependent on autophagy for growth and survival

Chapter $7 \quad$ General discussion and summary

Nederlandse samenvatting

Acknowledgements / Dankwoord

Curriculum Vitae 
<smiles>C=C</smiles> 


\section{Outline of the thesis}




\section{Outline of the thesis}

EGFR overexpression and mutations in the EGFR have significant impact on tumor growth, treatment response, and patient survival, as outlined in chapter 1. Currently, EGFR deregulated tumors are treated with TKIs or monoclonal antibodies like Cetuximab. Cetuximab is designed to block ligand binding of the EGFR and binds domain 1 and 3 of this receptor. EGFRvIII, a commonly found mutation variant of EGFR, lacks the majority of domain 1 and 2. Despite this truncation we hypothesized that Cetuximab can also bind to EGFRvIII and can thereby be used to treat EGFRvIll expressing tumors. Chapter 2 describes the binding of Cetuximab to EGFRvIll and its biologicals consequences in vitro.

Currently, EGFR expression levels, mutations, and its downstream effectors like k-RAS and PTEN are routinely evaluated for diagnosis and clinical treatment of patients. However, EGFRvIII is not routinely determent despite its clinical relevance ${ }^{1,2}$ and its adverse effect on anti-EGFR therapy. ${ }^{3}$ We therefore attempted to develop an EGFRvIII specific llama derived antibody suitable for in vitro and in vivo usage (chapter 3). Llama nanobodies (single domain heavy chain only antibody fragments) are in general suitable for both PET-imaging ${ }^{4}$ and detection on cryosections. ${ }^{5}$ The selected antibody had a high affinity for EGFRvIII but unfortunately also for wtEGFR.

In chapter $\mathbf{4}$ en $\mathbf{5}$ the consequences of EGFR and EGFRvIII expression in vitro and in vivo were investigated to elucidate the biological relevance of their expression. Interestingly, EGFRvIII expressing cells were more resistant to stresses like serum starvation and hypoxia in vitro and EGFRvIll expressing tumors grew faster. We hypothesized that EGFRvIll expressing cells and tumors require autophagy for their enhanced growth and survival. Indeed, EGFRvIll expressing cells growth and survival advantage could be abrogated by autophagy inhibition. Additionally, radio resistant EGFRvIll expressing tumors could be radio sensitized through autophagy inhibition. Moreover, in a cohort of glioblastoma patients, conventional therapy supplemented with chloroquine improved the overall survival of these patients. Interestingly, this effect was even more pronounced in patients with an EGFRvIll positive tumor. In chapter 6 we assessed the relation between EGFR expression and autophagy. A positive correlation between EGFR and LC3b protein and mRNA 
was observed in a panel of primary Head and Neck Squamous cell Carcinoma xenografts (HNSCC). Additionally, EGFR overexpressing glioma xenografts showed a reduction in growth when treated with chloroquine. Although in most tumors LC3b is predominantly expressed in hypoxic regions, LC3b expression in EGFR expressing tumors was predominantly located within the non-hypoxic regions. In contrast to non-EGFR expressing tumors, chloroquine administration lead to a large reduction in tumor growth suggesting that not only hypoxic cells, but also other cells that depend on autophagy, e.g. nutrient deprived cells are targeted. Taken together, inhibition of autophagy could therefore be explored as a novel treatment opportunity for EGFR and EGFRvIII overexpressing tumors.

Pelloski CE, Ballman KV, Furth AF, et al. Epidermal growth factor receptor variant III status defines clinically distinct subtypes of glioblastoma. J Clin Oncol 2007;25:22882294.

2 Shinojima N, Tada K, Shiraishi S, et al. Prognostic value of epidermal growth factor receptor in patients with glioblastoma multiforme. Cancer Res 2003;63:6962-6970.

3 Learn CA, Hartzell TL, Wikstrand CJ, et al. Resistance to tyrosine kinase inhibition by mutantepidermal growth factor receptor variant III contributes to the neoplastic phenotype of glioblastoma multiforme. Clin Cancer Res 2004;10:3216-3224.

4 Vosjan MJ, Perk LR, Roovers RC, et al. Facile labelling of an anti-epidermal growth factor receptor Nanobody with $68 \mathrm{Ga}$ via a novel bifunctional desferal chelate for immuno-PET. Eur J Nucl Med Mol Imaging 2011;38:753-763. van Koningsbruggen $\mathrm{S}$, de Haard $\mathrm{H}$, de Kievit $\mathrm{P}$, et al. Llama-derived phage display antibodies in the dissection of the human disease oculopharyngeal muscular dystrophy. J Immunol Methods 2003;279:149-161. 
<smiles>C=C</smiles> 


\section{Chapter 1}

General introduction

Published in: Cell Cycle 2014 Jan;13(1): 42-51

EGFR signaling and autophagy dependence for growth, survival, and therapy resistance

B.Jutten and K.M.A.Rouschop 


\section{Abstract}

The epidermal growth factor receptor (EGFR) is amplified or mutated in various human epithelial tumors. Its expression and activation leads to cell proliferation, differentiation, and survival. Consistently, EGFR amplification or expression of EGFR variant 3 (EGFRvIII) is associated with resistance to conventional cancer therapy through activation of pro-survival signaling and DNA-repair mechanisms. EGFR targeting has successfully been exploited as strategy to increase treatment efficacy. Nevertheless, these targeting strategies have only been proven effective in a limited percentage of human tumors.

Recent knowledge indicates that EGFR deregulated tumors display differences in autophagy and dependence on autophagy for growth and survival and the use of autophagy to increase resistance to EGFR-targeting drugs. In this review the dependency on autophagy and its role in mediating resistance to EGFRtargeting agents will be discussed. Considering the current knowledge, auto-phagy inhibition could provide a novel strategy to enhance therapy efficacy in treatment of EGFR deregulated tumors. 


\section{Introduction}

The epidermal growth factor receptor (EGFR) is a receptor tyrosine kinase within the ErbB family consisting of 4 members; EGFR (ErbB1, HER1), ErbB2 (HER2), ErbB3 (HER3), and ErbB4 (HER4). ErbBs are typical receptor tyrosine kinases that were implicated in cancer in the early 1980s, when the avian erythroblastosis tumor virus was found to encode an aberrant form of the human epidermal growth factor receptor. ${ }^{1}$ In many different cancer cell types, the ErbB pathway becomes hyperactivated by a range of mechanisms, including overproduction of ligands, overproduction of receptors, or constitutive activation of receptors. ${ }^{2}$ In general, EGFR signaling is triggered by ligand binding to the extracellular ligand binding domain. This initiates receptor homo/hetero-dimerization and autophosphorylation through the intracellular kinase domain, resulting in receptor activation. Following activation, cytoplasmic substrates are phosphorylated and initiate a signaling cascade that drives multiple cellular responses, including changes in gene expression, cytoskeletal rearrangement, apoptosis inhibition, and increased cell proliferation..$^{3,4}$ In cancer, EGFR signaling is often deregulated, leading to treatment resistance of the tumor and poor survival of patients. This deregulation is often mediated by overexpression (e.g., through gene amplification) and numerous mutations that lead to uncontrolled and sustained EGFR-signaling. Several EGFR targeting therapies have been developed (e.g., tyrosine kinase inhibitors (TKI) that inhibit EGFR signaling and antibodies that prevent EGFR expression and dimerization). Unfortunately, these therapies have only been proven effective in a limited percentage of cancer patients despite the presence of EGFR in many of the targeted tumors. ${ }^{5}$ Novel strategies that, potentially combined with earlier EGFR-targeting agents, lead to enhanced cell killing are therefore still desired.

Current research has indicated that EGFR-deregulated cells and tumors display alterations in their autophagic response, a pro-survival mechanism that allows cells to recycle nutrients for energy- and macromolecule production. ${ }^{6}$ Importantly: (1) EGFRderegulated cells seem to be more dependent on autophagy for growth and survival; and (2) resistance to EGFR-targeting agents can be reduced through autophagy inhibition, providing a potential novel 
modality to target these tumors. In this review we highlight current knowledge that may provide insights as to why EGFR-deregulated cells display differences in autophagic responses and dependency on autophagy for survival and provide rationale for combining autophagy inhibition with conventional cancer therapy.

\section{Gene Amplification and Overexpression}

One of the most investigated alterations in the EGFR function is activation of signaling through increased gene copy number arising from amplification or polysomy. ${ }^{7-9}$ Elevated EGFR expression is a strong prognostic indicator in head and neck, ovarian, cervical, bladder, and esophageal cancer. In gastric, breast endometrial, and colorectal cancers (CRC) EGFR expression is a modest predictor. This in contrast to non-small cell lung carcinoma (NSCLC), where increased EGFR expression rarely has a prognostic value. ${ }^{10}$

EGFR mutations often determine the responsiveness of tumors to EGFR inhibitors; this is often related to the dependency of cancer on continued oncogenic signaling (oncogene addiction). For a number of different oncogenes, data supporting addiction in tumors have been gathered. ${ }^{11,12}$ For EGFR in particular, positive results in clinical trials with different antagonists have been considered as clinical evidence of oncogene addiction, even though the clinical benefits from the use of either monoclonal antibodies (mAbs) or TKIs have been limited. ${ }^{5}$ Only a small portion (9-20\%) of tumors with hyperactive EGFR signaling is exquisitely sensitive to such specific inhibitors. ${ }^{13-}$ ${ }^{15}$ This percentage is much higher (88-94.1\%) when sensitizing mutations (e.g., L858R) in the EGFR gene are present. ${ }^{16,17}$ In NSCLC and CRC, increased EGFR gene copy number has been associated with increased clinical efficacy of EGFR antagonists erlotinib and cetuximab. ${ }^{18}$ Both drugs have shown clinical promise, and the anti-EGFR antibody cetuximab is used in treatment of head and neck squamous cell cancer (HNSCC) and CRC. Despite clinical gain, both intrinsic resistance and the development of acquired resistance have been observed..$^{19}$ 


\section{The Tyrosine Kinase Domain}

Both mutations associated with drug resistan,ce and sensitivity have been described within the tyrosine kinase (TK) domain of EGFR in subsets of NSCLC, rare cases in HNSCC, CRC, small cell lung carcinomas (SCLC), ovarian, esophageal, and pancreatic cancers. ${ }^{20}$ Distribution of mutations is not random and may be related to cancer etiology. For instance, in NSCLC the incidence of EGFR mutations among clinical responders to gefitinib or erlotinib is $77 \%$, compared with only $7 \%$ in NSCLC cases that are refractory to tyrosine kinase inhibitor (TKI) treatment. ${ }^{20}$ Multiple studies have shown differences in treatment outcome associated with EGFR mutations. For example, mutations in exon 18 (nucleotide-binding loop), accounting for $5 \%$ of the mutations, are usually amino acid substitutions that contribute to drug sensitivity. Mutations in exon 19 are characterized by small in-frame deletions and account for $45 \%$ of EGFR mutations, making it the most prominent EGFR kinase domain mutation in NSCLC. These tumors are, in general, sensitive to TKIs like gefitinib and erlotinib. ${ }^{20}$ The L858R substitution in exon 21, within the activation loop of EGFR, comprises approximately $40-45 \%$ of EGFR mutations. Tumors harboring the L858R mutation are, in general, sensitive to TKIs, although some clinical studies have shown that these tumors are not as responsive in comparison to tumors with deletion mutations in exon $19 .{ }^{20}$

EGFR exon 20 mutations, typically located after the C-helix of the tyrosine kinase domain, may account for up to $4 \%$ of all EGFR mutations, with the T790M substitution as the most prominent one (up to $50 \%$ of all mutations in exon 20). This T790M mutation is considered an acquired mutation and converts TKIsensitive tumors into (reversible) TKI-resistant tumors. ${ }^{21}$ Like the T790M mutation, other exon 20 mutated proteins are resistant to clinically achievable doses of reversible (gefitinib, erlotinib) and irreversible (neratinib, afatinib, PF00299804) TKIs in preclinical models. ${ }^{22}$ Growing clinical experience with tumors harboring EGFR exon 20 insertions correspond with the preclinical data; only few patients have shown responsiveness to EGFR TKIs. ${ }^{22}$ 


\section{EGFRvIII}

In a significant proportion of tumors, amplification of the EGFR gene is accompanied by rearrangements, although amplification is not mandatory for gene rearrangement. ${ }^{23}$ The most abundant rearrangement is a deletion variant that lacks exon 2-7 of the extracellular domain, yielding a constitutively active receptor, EGFRvIll or $\Delta 2-7 .{ }^{24-26}$ This mutation is most prevalent in malignant gliomas $(20-30 \%$ in unselected patients with a glioblastoma multiforme [GBM] and $50-60 \%$ in patients whose tumors show amplification of wild-type EGFR). ${ }^{27}$ Recent studies identified EGFRvIII in head and neck squamous cell carcinomas $(\sim 21 \%),{ }^{28}$ squamous cell carcinomas of the lung ( $\left.\sim 5 \%\right),{ }^{29,30}$ and breast $(\sim 5 \%),{ }^{31}$ suggesting broader implications to human cancer. ${ }^{32}$ EGFRvIII is known to contribute to radio resistance of tumor cell ${ }^{33}$ at least in part through enhanced repair of DNA doublestrand breaks. ${ }^{34}$ Additionally, EGFRvIII expression is associated with resistance to gefitinib and leads to sustained EGFR signaling and AKT activity. ${ }^{35}$ Furthermore, the tumor microenvironment, and in particular tumor hypoxia, significantly contributes to therapy resistance. ${ }^{36-38}$ Expression of EGFRvIll provides cells with a survival advantage when exposed to stresses such as hypoxia and nutrient starvation. ${ }^{39}$ Although EGFRvIll expression is frequently observed in GBM, culturing GBM cells in vitro will lead to a rapid loss of EGFRvIII expression, ${ }^{40,41}$ and thus complicates assessment of EGFRvIIItargeting strategies in GBM. Researchers therefore often use cell lines that artificially express EGFRvIII. Although informative, these cell lines have their limitations as, unlike in primary GBM (a range of 1-100\% EGFRvIll positive cells in GBM is observed), ${ }^{23}$ all cells will express EGFRvIll. Furthermore, the heterogeneous expression levels observed in GBM are difficult to mimic due to the use of artificial promoters; in addition, the cells were established without EGFRvIII and are thus not dependent on EGFRvIII for growth and survival. To maintain EGFRvIll expression in vitro, cells could be cultured under stem cell culture conditions. ${ }^{42}$ Alternatively, EGFRvIll expression is also maintained when primary tumors are xenografted subcutaneously on mice ${ }^{42}$ and should be considered in validating results obtained in transgenic models.

For GBM patients, EGFR overexpression is a significant prognostic value for predicting survival, and the expression of EGFRvIll with EGFR amplification 
plays an important role in enhanced tumorigenicity. EGFRvIII overexpression in the presence of EGFR amplification is the strongest indicator for poor survival prognosis in 2 large cohorts of patients. Shinojima and colleagues found in a cohort of 87 patients that EGFRvIll expression, assessed by immunohistochemistry (IHC), was not a predictor for overall survival (OS). However, in patients with EGFR amplification, multivariate analysis revealed that EGFRvIll expression was an independent, significant, poor prognostic factor for OS $(P=0.0044, H R=2.71) .{ }^{23}$ These findings were endorsed by Pelloski et al., ${ }^{43}$ who observed that the median survival of a patient group with EGFRvIII expression ( $n=36$, assessed by IHC) was reduced from 85 to $47 \mathrm{wk}$ compared with EGFRvIll-negative patient group $(n=81)$. In contrast, Montano et al. ${ }^{44}$ showed, in a cohort of 73 patients, that EGFRvIll (assessed by reverse transcription-PCR) is a molecular predictor of improved overall survival $(P=$ $0.0023, \mathrm{HR}=2.59$ ) in GBM patients treated with surgery followed by adjuvant radiotherapy and temozolomide (TMZ). This discrepancy could potentially be explained by the EGFRvIll detection method. Montano used the more sensitive RT-PCR, whereas Pelloski and Shinojima used IHC and may have missed very low levels of EGFRvIll expression. Another possible explanation for the differences could be the uniformness of the patient group. Montano used patients that all underwent surgery, radiotherapy, and TMZ treatment, whereas the other cohorts were treated more heterogeneously. Furthermore, all patients in Pelloski's study were wild-type for YKL-40 (a Ras activator), were Montano does not discriminate between Ras activator status, and the Karnofsky performance status (KPS score) of the patients in Pelloski's and Shinojima's cohort was much higher. ${ }^{23,43,44}$ Taken together, more and lager cohorts with uniform treatment are required to gain additional insight in the clinical relevance of EGFRvIII.

\section{Cancer Stem Cells}

Recent data showed that EGFR and EGFRvIII signaling are involved in maintaining a cancer stem cell (CSC) phenotype. In glioblastoma, both the EGFRpos and EGFRneg tumor-initiating cells (TICs) derived from primary GBM can give rise to experimental tumors. However, the EGFRpos TICs displayed 
enhanced tumorigenic potential and highly invasive behavior. ${ }^{45}$ Conversely, EGFRneg TICs formed tumors with low efficiency and needed to re-upregulate their EGFR expression to become tumorigenic. These "potential" CSCs might be kept in a dormancy-like state by EGFR downregulation and be reactivated when exposed to stimuli of the in vivo tumor microenvironment. ${ }^{45}$ Indeed, GBMs that were EGFRneg in origin expressed EGFR on recurrence. ${ }^{46}$ The idea of EGFRpos and EGFRneg CSC is further supported by the finding that CSC propagation is possible in the absence of exogenous growth factors (like EGF), suggesting that EGF signaling is not critical for GBM CSC maintenance. ${ }^{47}$ In contrast, EGFR signaling is required for GMB CSC proliferation, ${ }^{48,49}$ and gefitinib treatment decreases CSC number in nasopharyngeal carcinoma models. ${ }^{50}$ In this study, cisplatin-treated tumor cells regrew rapidly upon re-implantation, whereas regrowth of gefitinib-treated tumor cells was severely diminished..$^{50}$ Furthermore, Clark et al. ${ }^{51}$ showed that GBM CSC lines displayed tumorinitiating capacity after EGF withdrawal or cetuximab treatment by compensatory activation of ErbB2 and ErbB3, suggesting a resistance mechanism for EGFR-targeted therapy. Lapatinib, a dual EGFR/ErbB2 inhibitor, treatment inhibited CSCs proliferation, indicating that a simultaneous blockade of multiple ErbB family members could be needed for more efficient GBM treatment.

In relation to EGFRvIII in CSC, a population of the cells derived from pediatric diffuse intrinsic pontine gliomas (DIPG) neurospheres displayed co-expression of the CSC marker CD133 and EGFRvIII. ${ }^{52}$ In another study, EGFRvIII expression on invasive breast cancer carcinomas resulted in increased expression of genes related to self-renewal and epithelial-mesenchymal transition, along with a higher percentage of CSC-like cells. ${ }^{31}$ Furthermore, Liu et al. ${ }^{53}$ showed that the CD133+ fraction of GBM exclusively expressed EGFRvIII, whereas wild-type EGFR was not detected. These data indicate a role for EGFRvIII in the propagation of CSC that could explain the relative therapy resistance of EGFRvIII tumors.

\section{EGFR Signaling Pathways Implicated in Autophagy}

After ligand binding by EGFR or constitutive signaling by EGFRvIII the 
activation of several parallel pathways has been described. These include: (1) activation of the PI3K-AKTmTOR pathway; (2) increased Ras and (3) STAT3 signaling; and (4) Beclin1 (Fig. 1). ${ }^{54}$ All pathways involved in autophagy regulation.

Autophagy is a catabolic process that allows cells to recycle cellular components through degradation by the lysosomal machinery. ${ }^{55,56}$ Autophagy is an evolutionarily conserved process that results in the targeting of cellular proteins and organelles to lysosomes for degradation. Autophagy serves to regulate normal organelle turnover and the removal of those with compromised function to maintain cellular homeostasis. Additionally, autophagy is a survival mechanism during periods of metabolic stress, where self-digestion provides an alternative energy source and facilitates the disposal of unfolded proteins. ${ }^{57-}$ 60

Previously, we and others showed that cells with deregulated EGFR signaling display differences in autophagic response. ${ }^{61-63}$ Interestingly, EGFR expression represses autophagy activity. For example, EGFR reduction by siRNA treatment leads to an induction of autophagy activity in prostate cancer cells. ${ }^{63}$ Furthermore, induction in autophagy was observed after targeting with TKIs or cetuximab. ${ }^{64}$ Recently, in a panel of HNSSC xenografts, we observed a correlation between EGFR and expression of the autophagy marker Lc3b,
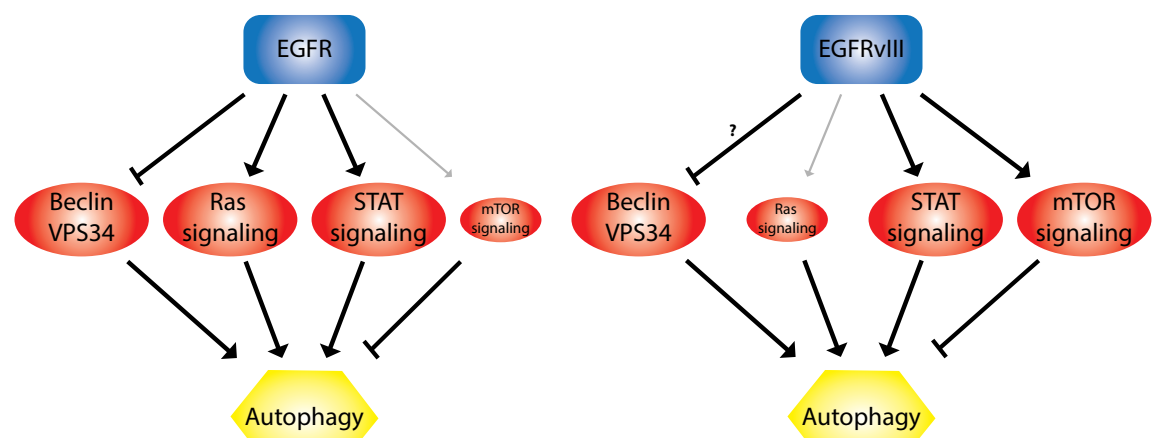

Figure 1. EGFR- and EGFRvIII-signaling pathways associated with auophagy regulation. Both receptors signal through all 4 pathways; nevertheless, EGFR preferentially signals via the RAS pathway, whereas EGFRvIll predominantly uses mTOR signaling. 
suggesting a close interplay between EGFR signaling and autophagy. This correlation is most likely mediated through controlling Lc3b protein production, as this correlation was also observed on the mRNA level. ${ }^{61}$ This was further confirmed in a panel of cell lines, where EGFR expression negatively correlated with autophagic flux, as determined by Lc3b-turnover. Interestingly, the suppressive activity of EGFR in these cells can be independent of its kinase activity $^{65}$ and mediated through maintaining high glucose levels through association with sodium/glucose cotransporter 1 (SGLT1). ${ }^{63}$ In addition, EGFR can suppress autophagy dependent on its kinase domain through maintaining high activation of the PI3K/Akt/mTOR pathway. ${ }^{66}$ Furthermore, EGFR activity results in inhibition of autophagy through inhibition of beclin $1,{ }^{62}$ a potent inducer of autophagy. Together these data indicate that the expression of EGFR is closely related to expression of autophagic markers and autophagic activity of cells.

Although the effect of EGFR seems to be mostly autophagysuppressive, in constitutive EGFR-signaling cells the effect on autophagy activity is less pronounced during normal conditions and seems to be stimulatory during metabolic stresses. For example, in stably transduced glioblastoma cell lines and prostate cancer cells that express EGFRvIII, a faster and more pronounced autophagic response during starvation or severe hypoxia is observed (unpublished data). The enhanced autophagic response provides these cells with survival and growth advantage.

The suppressive action of EGFR on autophagy activity and the opposing action of EGFRvIII during stressful conditions could result from signaling via different signal-transduction pathways. For example, Wolf-Yadlin et al. ${ }^{67}$ showed that EGFR predominantly signals via Erk1, Erk2, and STAT3, whereas EGFRvIII favors signaling via the PI3K and STAT3 pathway. ${ }^{68,69}$ This difference in signaling preference of these pathways associated with autophagy activity is likely to result in differences between EGFR and EGFRvIII.

\section{EGFR-PI3K-AKT-mTOR Pathway}

Activated EGFR binds GRB2-associated binding protein 1 (GAB1) together with growth factor receptorbound protein 2 (GRB2) to recruit phosphoinositide-3- 
kinase (PI3K). PI3K phosphorylates PI(4,5)P2 (phosphatidylinositol) into $\mathrm{PI}(3,4,5) \mathrm{P} 3$. This process is negatively regulated by phosphatase and tensin homolog (PTEN). 3-phosphoinositide dependent protein kinase-1 (PDK1) brings v-akt murine thymoma viral oncogene homolog 1 (AKT) to the plasma membrane, where PIP3 is located, to phosphorylate and activate AKT. AKT subsequently activates mTOR (mammalian target of Rapamycin). ${ }^{54}$ mTOR, a central growth regulator downstream of oxygen, energy, nutrient, and growth factor signaling, inhibits autophagy. Hence, insufficiency in either results in mTOR inhibition and rapid induction of autophagy in most systems. In conditions of nutrient sufficiency, high mTOR activity prevents Unc-51-like kinase (ULK1) activation by phosphorylating ULK1 Ser757 and disrupting the interaction between ULK1 and 5' AMP-activated protein kinase (AMPK), thereby preventing ULK1 to initiate an autophagy activating complex with FIP200 and ATG13. ${ }^{70,71}$ During periods of starvation, mTOR dissociates from the ULK1 complex, leading to less ULK1 phosphorylation, and increases ULK1 kinase activity. ${ }^{72,73}$ Recently, a role for ULK1 activation for survival of hypoxic cells was identified..$^{74,75}$

\section{EGFR-RAS Signaling Pathway}

The RAS oncogene is a member of small GTPase family involved in the regulation of cell survival and growth and is frequently activated in cancer. ${ }^{76}$ Next to frequently detected activating mutations in RAS, growth factor signaling, e.g., through EGFR, can lead to uncontrolled RAS signaling. After autophosphorylation, the adaptor protein growth factor receptor-bound protein 2 (GRB2) binds EGFR at the phosphorylated sites and activates Son of sevenless (SOS), a GTP-exchange factor for RAS. SOS then converts RASGDP into active RAS-GTP. Several studies have implicated RAS activity in the induction of autophagy, as displayed by a high autophagic flux after oncogenic RAS transformation. ${ }^{77}$ Increased autophagy in these cells is required to sustain a high metabolic rate, to prevent accumulation of damaged mitochondria, reduce oxygen consumption, and to prevent metabolic substrate depletion. .7-79 $^{\text {7. }}$ In relation, autophagy inhibition in RAS transformed cells leads to enhanced cell killing during nutrient deprivation. ${ }^{77}$ Furthermore, it has been shown that 
RAS plays a role in regulating the redox state of the cell, and that constitutive production of ROS correlates with RAS-induced cell transformation ${ }^{80,81}$ and mediates autophagy induction through activation of protein kinase 8 (JNK) and subsequent upregulation of ATG5 and ATG7. ${ }^{80}$

\section{EGFR-STAT3 Signaling Pathway}

The third main signaling mediator downstream of activated EGFR is the signal transducer and activator of transcription (STAT3) protein. STAT3 belongs to a family of at least 7 transcription factors that share conservation in coiled-coil, SRC homology (SH2), and DNA-binding domains. ${ }^{82}$ STAT3 is a latent transcription factor present in the cytoplasm of cells. Phosphorylation at Y705, is mediated through activation of several transmembrane receptors, such as EGFR, ${ }^{83}$ and is required for transcriptional activity or transactivation of members of the Janus kinase (JAK) protein family. ${ }^{84}$ Phosphorylation leads to dimerization, nuclear translocation, DNA binding, and gene activation. ${ }^{85}$ Recently, STAT3 has been recognized as a new autophagy regulator through suppression of PKR. ${ }^{86}$ Shen et al..$^{86}$ proposed that in normal conditions, latent cytoplasmic STAT3 binds to protein kinase R (PKR), inhibiting its activity, and reduces autophagy levels through elF $2 \alpha$ inhibition, a signaling cascade involved in both transcriptional and translational regulation of Lc3b and ATG5 production. ${ }^{60}$ Hence, STAT3 phosphorylation leads to homodimerization and enables the free PKR to phosphorylate elF2 $\alpha$ via direct interaction between STAT3 and PKR. ${ }^{87}$ Furthermore, STAT3 controls the expression of several autophagy-associated proteins, including BCL-2, Bcl-XL, and MCL-1, ${ }^{88,89}$ which inhibit autophagy through sequestration of Beclin $1 .{ }^{60}$

\section{EGFR-Beclin 1}

Beclin 1 is a coiled-coil protein involved in the regulation of autophagy in mammalian cells and is a component of the class III phosphatidylinositol-3kinase (PI3K) complex. ${ }^{90}$ Beclin 1 promotes autophagy, and cells with reduced Beclin 1 expression exhibit reduced autophagic activity. ${ }^{91}$ Beclin 1 is an essential gene for early embryonic development and is a haploinsufficient tumor 
suppressor. ${ }^{92}$ Intriguingly, Beclin 1 is tumor suppressive in breast cancer cells; mice that have only one functional allele of Beclin 1 display higher incidence of spontaneous tumors, and mono-allelical deletions of Beclin 1 have been described for $40-75 \%$ of human ovarian, breast, and prostate cancers. ${ }^{91,93-95}$ Beclin 1 may also promote survival as an interacting partner of an anti-apoptotic protein Bcl-2. ${ }^{96}$ Binding of $\mathrm{Bcl}-2$ to Beclin 1 inhibits Beclin 1-dependent autophagy and Beclin 1-dependent autophagic cell death. ${ }^{91,97}$ Recently, it was shown that EGFR phosphorylates Beclin 1 at 3 different tyrosine residues, Y229, Y233, and Y352, after activation by EGF. This tyrosine phosphorylation favors the formation of Beclin 1 dimers, which are incapable of VPS34 binding, and results in reduced autophagy activation (Fig. 1). ${ }^{62}$

\section{EGFRvIII Tumors Require Increased Metabolism}

Why EGFRvIll-expressing tumors require higher activation of autophagy during metabolic stress remains unclear, but could be related to the higher proliferation rate and associated nutritional demand. For example, Guo et al ${ }^{98}$ showed that EGFRvIll expression induces major shifts in GBM cell metabolism. Uptake of 18FDG in EGFRvIll-expressing U87 xenografts was doubled compared with volume matched control xenografts. In relation, gene expression arrays showed upregulation of genes involved in regulation of the cell metabolism, e.g., glucose transporter 1 (GLUT1) and GLUT3, Hexokinase2 (HK2), and pyruvate dehydrogenase kinase (PDK1)..$^{99}$ In general, EGFRvlll-expressing tumors require upregulation of cell metabolism proteins and require increased glucose uptake to maintain their elevated growth rate. This might explain why these tumors may display increased dependence on autophagy for their energy supply in a tumor microenvironment that is low in glucose or deprived of oxygen.

\section{EGFR Mediates Mitochondrial Homeostasis}

In relation to the involvement of EGFR in cell metabolism, Rasbach et al. showed the involvement of EGFR in mitochondrial biogenesis after oxidant injury via EGFR-dependent p38 MAPK activation of the mitochondrial 
biogenesis regulator PPAR-y cofactor-1 $\alpha$ (PGC-1), ${ }^{100}$ allowing the cells to maintain high metabolism and their increased proliferation rate.

Additionally, EGFR is involved in stabilizing mitochondria and preventing apoptosis. Synergistic interaction between EGFR and c-Src via phosphorylation of EGFR at Y845 causes translocation to the mitochondria. There, it interacts with cytochrome c oxidase subunit II (COX II) and prevents apoptosis. This seems independent of EGFR kinase activity but is enhanced by EGF treatment. ${ }^{101,102}$ Although cells did not undergo apoptosis, ATP production was drastically reduced by binding of EGFR to COX II. ${ }^{102}$ Similar mechanisms and translocation to the mitochondria to antagonize apoptosis have been observed for EGFRvIII. ${ }^{103,104}$ COX II inhibition by EGFR leaves the cell with reduced ATP production after insults such as chemo- and radiotherapy or starvation and must revert to other sources for their ATP production. Autophagy may provide an alternative source for energy production, and could be exploited therapeutically in stressed cells with EGFR overexpression. This could also explain why EGFRexpressing cells are more dependent on autophagy for their survival. ${ }^{61}$

\section{EGFR, Treatment Resistance, and Therapeutic Potential of Autophagy Inhibition}

EGFR expression or mutations contribute to tumor treatment resistance. For instance, acquired mutations in the kinase domain of EGFR (like the T790M) can abrogate the susceptibility to TKIs like gefitinib or erlotinib. ${ }^{21}$ Furthermore, EGFR contributes to radiotherapy resistance either through activation of the pro-survival pathway PLCY-PKC-RAF ${ }^{105}$ or through activation of DNA repair via DNA-PK. ${ }^{106}$ We have also shown that expression of EGFRvIII contributes to stress resistance typical for the tumor microenvironment, including nutrient deprivation and hypoxia. ${ }^{39}$ Hypoxia is a common feature of tumors and an important contributor to malignancy and treatment resistance, ${ }^{36,37,107}$ and in HNSCC, the degree of hypoxia is the most significant factor explaining variability in survival. ${ }^{37}$ Targeting hypoxia in pre-clinical models has been shown to sensitize tumors to therapy through various modalities. ${ }^{60,108,109}$ Importantly, a metaanalysis in HNSCC demonstrated therapeutic benefit of hypoxia modification. ${ }^{110}$ Tumor cells adapt to hypoxia through multiple mechanisms, 
including activation of autophagy.,60,111-115 Genetic and pharmacological inhibition of autophagy sensitizes human tumor cells to hypoxia, reduces the fraction of viable hypoxic tumor cells, and sensitizes human tumors xenografts to irradiation (Fig. 2A) ${ }^{60}$ In relation to EGFR expression, although we showed reduced autophagic flux in cells expressing EGFR, these cells were already under normal conditions dependent on autophagy for proliferation and survival. ${ }^{61}$ In general, EGFR-expressing tumors are considered highly radioresistant; ${ }^{116}$ also in our setting, a large dose irradiation had only a minor effect on tumor delay. Interestingly, chloroquine administration to inhibit autophagy led to a large delay in tumor growth that exceeded the effect of irradiation and, in addition, sensitized tumors to irradiation. ${ }^{61}$

A

Normal tumor

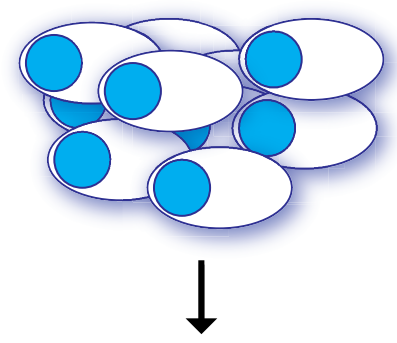

Autophagy inhibition

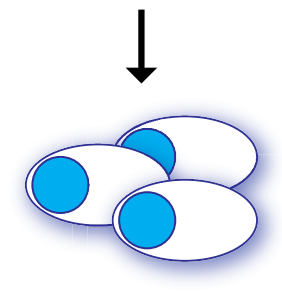

B

Anti EGFR treatment resistant tumor

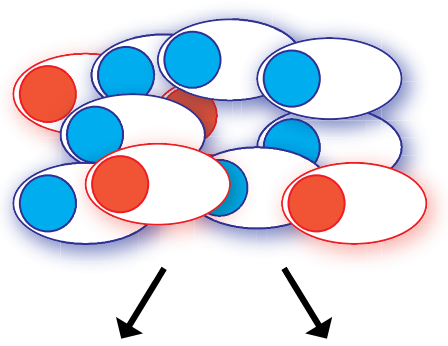

mAbs TKIs

Autophagy Inhibition

$$
\begin{aligned}
& + \\
& \text { mAbs }
\end{aligned}
$$

TKIs

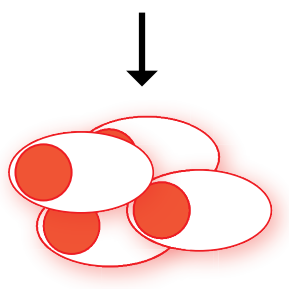

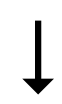

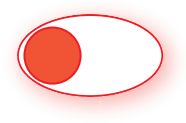

Figure 2. (A) In EGFR-deregulated tumors, inhibition of autophagy leads to increased cell killing of metabolic stressed cells. (B) Resistance of tumor cells with active EGFR signaling to monoclonal antibodies (mAbs) or tyrosine kinase inhibitors (TKIs) can be reduced by autophagy inhibition. 
Recent data showed that EGFR and EGFRvIII signaling is involved in maintaining a CSC phenotype, and recently it was shown that autophagy is important for CSC self-renewal and tumorigenic potential in breast cancer stem cells, ${ }^{117}$ and for regulation of energy metabolism and migration and invasion of GBM-derived stem cells. ${ }^{118}$ Taken together, these data suggest that autophagy and EGFR or EGFRvIll signaling are very important in CSC and could therefore be considered for dual targeted therapy for treatment of CSCs in patients. Why EGFRand EGFRvIll-expressing cells and tumors are more sensitive to chloroquine treatment remains matter of investigation.

Clinical efficacy of anti EGFR drugs to date has been limited by both acquired and intrinsic resistance. Cancer cells adapt rapidly to EGFR inhibition treatment, resulting in only a small success rate for EGFR inhibition as mono therapy in cancer treatment ${ }^{119,120}$ (Fig. 2B). Nevertheless, inhibition of EGFR signaling in combination with autophagy inhibition looks promising in vitro. In NSCLC cell lines with activating EGFR mutation (exon 19 deletion) erlotinib induces both apoptosis and autophagy. Inhibition of autophagy can further enhance sensitivity to erlotinib in these NSCLC cells, suggesting that autophagy serves as a protective mechanism. ${ }^{121}$ Moreover, wild-type EGFR-expressing NSCLC cells' resistance to erlotinib can be abrogated through autophagy inhibition. ${ }^{122}$ Furthermore, ZD6474, a small molecule inhibitor of VEGFR, EGFR, and RET induces apoptosis in 2 glioblastoma cell lines, which can be enhanced by the inhibition of autophagy. ${ }^{123}$ These findings suggest a therapeutic opportunity for the inhibition of autophagy in combination with conventional cancer therapies.

\section{Conclusion}

Over the last decades EGFR has evolved as highly investigated target in the field of anti-cancer treatment. This has led to the development of EGFRtargeting antibodies like cetuximab or panitumumab and TKIs like gefitinib, erlotinib, and lapatinib. More recently, the potential of autophagy inhibition as therapy in cancer is being evaluated. Several reports indicate that cells and tumors with amplified or overactivated EGFR are particularly sensitive to autophagy inhibition for growth, survival, and resistance to conventional therapies. Additionally, resistance to EGFR-targeting therapies can also be 
reduced by autophagy inhibition. Inhibition of autophagy may therefore provide a novel treatment opportunity for EGFR-overexpressing tumors and should be pursued clinically.

\section{Acknowledgments}

This work was financially supported by the Dutch Cancer Society (KWF Grants UM 2010-4714 and 2012-5506), foundations "STOPhersentumoren" and "Zeldzame Ziekten Fonds".

\section{References}

1. Downward J, Yarden Y, Mayes E, Scrace G, Totty N, Stockwell P, Ullich A, Schlessinger $\mathrm{J}$, Waterfield MD. Close similarity of epidermal growth factor receptor and v-erb-B oncogene protein sequences. Nature 1984; 307:521-7

2. Yarden Y, Sliwkowski MX. Untangling the ErbB signalling network. Nat Rev Mol Cell Biol 2001; 2:127-37

3. Lemmon MA, Bu Z, Ladbury JE, Zhou M, Pinchasi D, Lax I, Engelman DM, Schlessinger J. Two EGF molecules contribute additively to stabilization of the EGFR dimer. EMBO J 1997; 16:281- 94

4. Lingi $B$, Carpenter $G$. ErbB receptors: new insights on mechanisms and biology. Trends Cell Biol 2006; 16:649-56

5. Perez R, Crombet T, de Leon J, Moreno E. A view on EGFR-targeted therapies from the oncogeneaddiction perspective. Front Pharmacol 2013; 4:53

6. Mizushima N. The pleiotropic role of autophagy: from protein metabolism to bactericide. Cell Death Differ 2005; 12(Suppl 2):1535-41

7. King CR, Kraus MH, Aaronson SA. Amplification of a novel v-erbB-related gene in a human mammary carcinoma. Science 1985; 229:974-6

8. King CR, Kraus MH, Williams LT, Merlino GT, Pastan IH, Aaronson SA. Human tumor cell lines with EGF receptor gene amplification in the absence of aberrant sized mRNAs. Nucleic Acids Res 1985;13:8477-86

9. Libermann TA, Nusbaum HR, Razon N, Kris R, Lax I, Soreq H, Whittle N, Waterfield MD, Ullrich A, Schlessinger J. Amplification, enhanced expression and possible rearrangement of EGF receptor gene in primary human brain tumours of glial origin. Nature 1985;313:144-7

10. Nicholson RI, Gee JM, Harper ME. EGFR and cancer prognosis. Eur J Cancer 2001; 37(Suppl 4):S9-15 
11. Weinstein IB, Joe A. Oncogene addiction. Cancer Res 2008; 68:3077-80, discussion 3080

12. Weinstein IB, Joe AK. Mechanisms of disease: Oncogene addiction--a rationale for molecular targeting in cancer therapy. Nat Clin Pract Oncol 2006; 3:448-57

13. Cunningham D, Humblet $Y$, Siena S, Khayat D, Bleiberg H, Santoro A, Bets D, Mueser M, Harstrick A, Verslype $C$, et al. Cetuximab monotherapy and cetuximab plus irinotecan in irinotecan-refractory metastatic colorectal cancer. N Engl J Med 2004; 351:337-45

14. Shepherd FA, Rodrigues Pereira J, Ciuleanu T, Tan EH, Hirsh V, Thongprasert S, Campos D, Maoleekoonpiroj S, Smylie M, Martins R, et al.; National Cancer Institute of Canada Clinical Trials Group. Erlotinib in previously treated non-smallcell lung cancer. N Engl J Med 2005; 353:123-32

15. Tsao MS, Sakurada A, Cutz JC, Zhu CQ, Kamel-Reid S, Squire J, Lorimer I, Zhang T, Liu N, Daneshmand M, et al. Erlotinib in lung cancer - molecular and clinical predictors of outcome. N Engl J Med 2005; 353:133-44

16. Lynch TJ, Bell DW, Sordella R, Gurubhagavatula S, Okimoto RA, Brannigan BW, Harris PL, Haserlat SM, Supko JG, Haluska FG, et al. Activating mutations in the epidermal growth factor receptor underlying responsiveness of non-small-cell lung cancer to gefitinib. N Engl J Med 2004; 350:2129-39

17. Taron M, Ichinose Y, Rosell R, Mok T, Massuti B, Zamora L, Mate JL, Manegold C, Ono $\mathrm{M}$, Queralt C, et al. Activating mutations in the tyrosine kinase domain of the epidermal growth factor receptor are associated with improved survival in gefitinib-treated chemorefractory lung adenocarcinomas. Clin Cancer Res 2005; 11:5878-85

18. Hirsch FR, Varella-Garcia M, Bunn PA Jr., Di Maria MV, Veve R, Bremmes RM, Barón $A E$, Zeng $C$, Franklin WA. Epidermal growth factor receptor in non-small-cell lung carcinomas: correlation between gene copy number and protein expression and impact on prognosis. J Clin Oncol 2003; 21:3798-807

19. Brand TM, lida M, Wheeler DL. Molecular mechanisms of resistance to the EGFR monoclonal antibody cetuximab. Cancer Biol Ther 2011; 11:777-92

20. Sharma SV, Bell DW, Settleman J, Haber DA. Epidermal growth factor receptor mutations in lung cancer. Nat Rev Cancer 2007; 7:169-81

21. Yun CH, Mengwasser KE, Toms AV, Woo MS, Greulich H, Wong KK, Meyerson M, Eck MJ. The T790M mutation in EGFR kinase causes drug resistance by increasing the affinity for ATP. Proc Natl Acad Sci U S A 2008; 105:2070-5

22. Yasuda H, Kobayashi S, Costa DB. EGFR exon 20 insertion mutations in non-small-cell lung cancer: preclinical data and clinical implications. Lancet Oncol 2012; 13:e23-31

23. Shinojima N, Tada K, Shiraishi S, Kamiryo T, Kochi M, Nakamura H, Makino K, Saya H, Hirano H, Kuratsu J, et al. Prognostic value of epidermal growth factor receptor in patients with glioblastoma multiforme. Cancer Res 2003; 63:6962-70

24. Wong AJ, Ruppert JM, Bigner SH, Grzeschik CH, Humphrey PA, Bigner DS, Vogelstein 
B. Structural alterations of the epidermal growth factor receptor gene in human gliomas. Proc Natl Acad Sci U S A 1992; 89:2965-9

25. Sugawa N, Ekstrand AJ, James CD, Collins VP. Identical splicing of aberrant epidermal growth factor receptor transcripts from amplified rearranged genes in human glioblastomas. Proc Natl Acad Sci U S A 1990

26. Yamazaki H, Fukui Y, Ueyama Y, Tamaoki N, Kawamoto T, Taniguchi S, Shibuya M. Am plification of the structurally and functionally altered epidermal growth factor receptor gene (c-erbB) in human brain tumors. Mol Cell Biol 1988; 8:1816-20

27. Gan HK, Kaye AH, Luwor RB. The EGFRvIll variant in glioblastoma multiforme. J Clin Neurosci 2009; 16:748-54

28. Smilek P, Neuwirthova J, Jarkovsky J, Dusek L, Rottenberg J, Kostrica R, Srovnal J, Hajduch M, Drabek J, Klozar J. Epidermal growth factor receptor (EGFR) expression and mutations in the EGFR signaling pathway in correlation with anti-EGFR therapy in head and neck squamous cell carcinomas. Neoplasma 2012; 59:508-15

29. Sasaki H, Kawano O, Endo K, Yukiue H, Yano M, Fujii Y. EGFRvIll mutation in lung cancer correlates with increased EGFR copy number. Oncol Rep 2007;17:319-23

30. Ji H, Zhao X, Yuza Y, Shimamura T, Li D, Protopopov A, Jung BL, McNamara K, Xia H, Glatt KA, et al. Epidermal growth factor receptor variant III mutations in lung tumorigenesis and sensitivity to tyrosine kinase inhibitors. Proc Natl Acad Sci U S A 2006; 103:7817-22

31. Del Vecchio CA, Jensen KC, Nitta RT, Shain AH, Giacomini CP, Wong AJ. Epidermal growth factor receptor variant III contributes to cancer stem cell phenotypes in invasive breast carcinoma. Cancer Res 2012; 72:2657-71

32. Moscatello DK, Holgado-Madruga M, Godwin AK, Ramirez G, Gunn G, Zoltick PW, Biegel JA, Hayes RL, Wong AJ. Frequent expression of a mutant epidermal growth factor receptor in multiple human tumors. Cancer Res 1995; 55:5536-9

33. Weppler SA, Li Y, Dubois L, Lieuwes N, Jutten B, Lambin P, Wouters BG, Lammering G. Expression of EGFR variant vIll promotes both radiation resistance and hypoxia tolerance. Radiother Oncol 2007; 83:333-9

34. Golding SE, Morgan RN, Adams BR, Hawkins AJ, Povirk LF, Valerie K. Pro-survival AKT and ERK signaling from EGFR and mutant EGFRvIll enhances DNA double-strand break repair in human glioma cells. Cancer Biol Ther 2009; 8:730-8

35. Learn CA, Hartzell TL, Wikstrand CJ, Archer GE, Rich JN, Friedman AH, Friedman HS, Bigner DD, Sampson JH. Resistance to tyrosine kinase inhibition by mutant epidermal growth factor receptor variant III contributes to the neoplastic phenotype of glioblastoma multiforme. Clin Cancer Res 2004; 10:3216-24

36. Hockel M, Schlenger K, Aral B, Mitze M, Schaffer U, Vaupel P. Association between tumor hypoxia and malignant progression in advanced cancer of the uterine cervix. Cancer Res 1996; 56:4509-15 
37. Nordsmark M, Bentzen SM, Rudat V, Brizel D, Lartigau E, Stadler P, Becker A, Adam M, Molls M, Dunst J, et al. Prognostic value of tumor oxygenation in 397 head and neck tumors after primary radiation therapy. An international multi-center study. Radiother Oncol 2005; 77:18-24

38. Wouters BG, van den Beucken T, Magagnin MG, Lambin P, Koumenis C. Targeting hypoxia tolerance in cancer. Drug Resist Updat 2004; 7:25-40

39. Theys J, Jutten B, Dubois L, Rouschop KM, Chiu RK, Li Y, Paesmans K, Lambin P, Lammering G, Wouters BG. The deletion mutant EGFRvIll significantly contributes to stress resistance typical for the tumour microenvironment. Radiother Oncol 2009; 92:399-404

40. Bigner SH, Humphrey PA, Wong AJ, Vogelstein B, Mark J, Friedman HS, Bigner DD. Characterization of the epidermal growth factor receptor in human glioma cell lines and xenografts. Cancer Res 1990; 50:8017-22

41. Pandita A, Aldape KD, Zadeh G, Guha A, James CD. Contrasting in vivo and in vitro fates of glioblastoma cell subpopulations with amplified EGFR. Genes Chromosomes Cancer 2004; 39:29-36

42. Stockhausen MT, Broholm H, Villingshøj M, Kirchhoff M, Gerdes T, Kristoffersen K, Kosteljanetz M, Spang-Thomsen M, Poulsen HS. Maintenance of EGFR and EGFRvIII expressions in an in vivo and in vitro model of human glioblastoma multiforme. Exp Cell Res 2011; 317:1513-26

43. Pelloski CE, Ballman KV, Furth AF, Zhang L, Lin E, Sulman EP, Bhat K, McDonald JM, Yung WK, Colman $\mathrm{H}$, et al. Epidermal growth factor receptor variant III status defines clinically distinct subtypes of glioblastoma. J Clin Oncol 2007; 25:2288-94

44. Montano N, Cenci T, Martini M, D’Alessandris QG, Pelacchi F, Ricci-Vitiani L, Maira G, De Maria R, Larocca LM, Pallini R. Expression of EGFRvIII in glioblastoma: prognostic significance revisited. Neoplasia 2011; 13:1113-21

45. Mazzoleni S, Politi LS, Pala M, Cominelli M, Franzin A, Sergi Sergi L, Falini A, De Palma M, Bulfone A, Poliani PL, et al. Epidermal growth factor receptor expression identifies functionally and molecularly distinct tumor-initiating cells in human glioblastoma multi forme and is required for gliomagenesis. Cancer Res 2010; 70:7500-13

46. Martinez R, Rohde V, Schackert G. Different molecular patterns in glioblastoma multiforme subtypes upon recurrence. J Neurooncol 2010; 96:321-9

47. Kelly JJ, Stechishin O, Chojnacki A, Lun X, Sun B, Senger DL, Forsyth P, Auer RN, Dunn JF, Cairncross JG, et al. Proliferation of human glioblastoma stem cells occurs independently of exogenous mitogens. Stem Cells 2009; 27:1722-33

48. Griffero F, Daga A, Marubbi D, Capra MC, Melotti A, Pattarozzi A, Gatti M, Bajetto A, Porcile C, Barbieri F, et al. Different response of human glioma tumorinitiating cells to epidermal growth factor receptor kinase inhibitors. J Biol Chem 2009; 284:7138-48

49. Soeda A, Inagaki A, Oka N, Ikegame Y, Aoki H, Yoshimura S, Nakashima S, Kunisada T, 
Iwama T. Epidermal growth factor plays a crucial role in mitogenic regulation of human brain tumor stem cells. J Biol Chem 2008; 283:10958-66

50. Ma L, Zhang G, Miao XB, Deng XB, Wu Y, Liu Y, Jin ZR, Li XQ, Liu QZ, Sun DX, et al. Cancer stem-like cell properties are regulated by EGFR/AKT/ß-catenin signaling and preferentially inhibited by gefitinib in nasopharyngeal carcinoma. FEBS J 2013;280:202741

51. Clark PA, lida M, Treisman DM, Kalluri H, Ezhilan S, Zorniak M, Wheeler DL, Kuo JS. Activation of multiple ERBB family receptors mediates glioblastoma cancer stem-like cell resistance to EGFRtargeted inhibition. Neoplasia 2012; 14:420-8

52. Li G, Mitra SS, Monje M, Henrich KN, Bangs CD, Nitta RT, Wong AJ. Expression of epidermal growth factor variant III (EGFRvIII) in pediatric diffuse intrinsic pontine gliomas. J Neurooncol 2012; 108:395-402

53. Liu XJ, Wu WT, Wu WH, Yin F, Ma SH, Qin JZ, Liu XX, Liu YN, Zhang XY, Li P, et al. A minority subpopulation of CD133(+)/EGFRvIII(+)/EGFR(-) cells acquires stemness and contributes to gefitinib resistance. CNS Neurosci Ther 2013; 19:494-502

54. Kolch W, Pitt A. Functional proteomics to dissect tyrosine kinase signalling pathways in cancer. Nat Rev Cancer 2010; 10:618-29

55. Edinger AL, Thompson CB. Defective autophagy leads to cancer. Cancer Cell 2003; 4:422-4

56. Levine B. Eating oneself and uninvited guests: autophagy-related pathways in cellular defense. Cell 2005; 120:159-62

57. Komatsu M, Waguri S, Ueno T, Iwata J, Murata S, Tanida I, Ezaki J, Mizushima N, Ohsumi Y, Uchiyama Y, et al. Impairment of starvation-induced and constitutive autophagy in Atg7-deficient mice. J Cell Biol 2005; 169:425-34

58. Kuma A, Hatano M, Matsui M, Yamamoto A, Nakaya H, Yoshimori T, Ohsumi Y, Tokuhisa $\mathrm{T}$, Mizushima $\mathrm{N}$. The role of autophagy during the early neonatal starvation period. Nature 2004; 432:1032-6

59. Degenhardt K, Mathew R, Beaudoin B, Bray K, Anderson D, Chen G, Mukherjee C, Shi Y, Gélinas C, Fan Y, et al. Autophagy promotes tumor cell survival and restricts necrosis, inflammation, and tumorigenesis. Cancer Cell 2006; 10:51-64

60. Rouschop KM, van den Beucken T, Dubois L, Niessen H, Bussink J, Savelkouls K, Keulers T, Mujcic H, Landuyt W, Voncken JW, et al. The unfolded protein response protects human tumor cells during hypoxia through regulation of the autophagy genes MAP1LC3B and ATG5. J Clin Invest 2010; 120:127-41

61. Jutten B, Keulers TG, Schaaf MB, Savelkouls K, Theys J, Span PN, Vooijs MA, Bussink J, Rouschop KM. EGFR overexpressing cells and tumors are dependent on autophagy for growth and survival. Radiother Oncol 2013; 108:479-83

62. Wei Y, Zou Z, Becker N, Anderson M, Sumpter R, Xiao G, Kinch L, Koduru P, Christudass $\mathrm{CS}$, Veltri RW, et al. EGFR-mediated Beclin 1 phosphorylation in autophagy suppression, 
tumor progression, and tumor chemoresistance. Cell 2013; 154:1269-84

63. Weihua Z, Tsan R, Huang WC, Wu Q, Chiu CH, Fidler IJ, Hung MC. Survival of cancer cells is maintained by EGFR independent of its kinase activity. Cancer Cell 2008; 13:38593

64. Li X, Fan Z. The epidermal growth factor receptor antibody cetuximab induces autophagy in cancer cells by downregulating HIF-1alpha and Bcl-2 and activating the beclin 1/hVps34 complex. Cancer Res 2010; 70:5942-52

65. Han W, Pan H, Chen Y, Sun J, Wang Y, Li J, Ge W, Feng L, Lin X, Wang X, et al. EGFR tyrosine kinase inhibitors activate autophagy as a cytoprotective response in human lung cancer cells. PLoS One 2011; 6:e18691

66. Li X, Lu Y, Pan T, Fan Z. Roles of autophagy in cetuximab-mediated cancer therapy against EGFR. Autophagy 2010; 6:1066-77

67. Wolf-Yadlin A, Hautaniemi S, Lauffenburger DA, White FM. Multiple reaction monitoring for robust quantitative proteomic analysis of cellular signaling networks. Proc Natl Acad Sci U S A 2007; 104:5860-5

68. Huang PH, Mukasa A, Bonavia R, Flynn RA, Brewer ZE, Cavenee WK, Furnari FB, White FM. Quantitative analysis of EGFRvIII cellular signaling networks reveals a combinatorial therapeutic strategy for glioblastoma. Proc Natl Acad Sci U S A 2007; 104:12867-72

69. Moscatello DK, Holgado-Madruga M, Emlet DR, Montgomery RB, Wong AJ. Constitutive activation of phosphatidylinositol 3-kinase by a naturally occurring mutant epidermal growth factor receptor. J Biol Chem 1998; 273:200-6

70. Alers S, Löffler AS, Wesselborg S, Stork B. Role of AMPK-mTOR-Ulk1/2 in the regulation of autophagy: cross talk, shortcuts, and feedbacks. Mol Cell Biol 2012; 32:2-11

71. Kim J, Kundu M, Viollet B, Guan KL. AMPK and mTOR regulate autophagy through direct phosphorylation of Ulk1. Nat Cell Biol 2011; 13:132-41

72. Hosokawa N, Hara T, Kaizuka T, Kishi C, Takamura A, Miura Y, lemura S, Natsume T, Takehana K, Yamada N, et al. Nutrient-dependent mTORC1 association with the ULK1Atg13-FIP200 complex required for autophagy. Mol Biol Cell 2009; 20:1981-91

73. Jung $\mathrm{CH}$, Seo M, Otto NM, Kim DH. ULK1 inhibits the kinase activity of mTORC1 and cell proliferation. Autophagy 2011; 7:1212-21

74. Pike LR, Singleton DC, Buffa F, Abramczyk O, Phadwal K, Li JL, Simon AK, Murray JT, Harris AL. Transcriptional up-regulation of ULK1 by ATF4 contributes to cancer cell survival. Biochem J 2013; 449:389-400

75. Schaaf MB, Cojocari D, Keulers TG, Jutten B, Starmans MH, de Jong MC, Begg AC, Savelkouls KG, Bussink J, Vooijs M, et al. The autophagy associated gene, ULK1, promotes tolerance to chronic and acute hypoxia. Radiother Oncol 2013; 108:529-34

76. Schubbert S, Shannon K, Bollag G. Hyperactive Ras in developmental disorders and cancer. Nat Rev Cancer 2007; 7:295-308

77. Guo JY, Chen HY, Mathew R, Fan J, Strohecker AM, Karsli-Uzunbas G, Kamphorst JJ, 
Chen G, Lemons JM, Karantza V, et al. Activated Ras requires autophagy to maintain oxidative metabolism and tumorigenesis. Genes Dev 2011; 25:460-70

78. Kim MJ, Woo SJ, Yoon CH, Lee JS, An S, Choi YH, Hwang SG, Yoon G, Lee SJ. Involvement of autophagy in oncogenic K-Ras-induced malignant cell transformation. J Biol Chem 2011; 286:12924-32

79. Lock R, Roy S, Kenific CM, Su JS, Salas E, Ronen SM, Debnath J. Autophagy facilitates glycolysis during Ras-mediated oncogenic transformation. Mol Biol Cell 2011; 22:16578

80. Alexandrova AY, Kopnin PB, Vasiliev JM, Kopnin BP. ROS up-regulation mediates Rasinduced changes of cell morphology and motility. Exp Cell Res 2006; 312:2066-73

81. Kopnin PB, Agapova LS, Kopnin BP, Chumakov PM. Repression of sestrin family genes contributes to oncogenic Ras-induced reactive oxygen species up-regulation and genetic instability. Cancer Res 2007; 67:4671-8

82. Ren Z, Mao X, Mertens C, Krishnaraj R, Qin J, Mandal PK, Romanowski MJ, McMurray JS, Chen X. Crystal structure of unphosphorylated STAT3 core fragment. Biochem Biophys Res Commun 2008; 374:1-5

83. Zhong Z, Wen Z, Darnell JE Jr. Stat3: a STAT family member activated by tyrosine phosphorylation in response to epidermal growth factor and interleukin-6. Science 1994; 264:95-8

84. Boulton TG, Zhong Z, Wen Z, Darnell JE Jr., Stahl N, Yancopoulos GD. STAT3 activation by cytokines utilizing gp130 and related transducers involves a secondary modification requiring an H7-sensitive kinase. Proc Natl Acad Sci U S A 1995; 92:6915-9

85. Levy DE, Darnell JE Jr. Stats: transcriptional control and biological impact. Nat Rev Mol Cell Biol 2002; 3:651-62

86. Shen S, Niso-Santano M, Adjemian S, Takehara T, Malik SA, Minoux H, Souquere S, Mariño G, Lachkar S, Senovilla L, et al. Cytoplasmic STAT3 represses autophagy by inhibiting PKR activity. Mol Cell 2012; 48:667-80

87. Niso-Santano M, Shen S, Adjemian S, Malik SA, Mariño G, Lachkar S, Senovilla L, Kepp O, Galluzzi L, Maiuri MC, et al. Direct interaction between STAT3 and EIF2AK2 controls fatty acid-induced autophagy. Autophagy 2013; 9:415-7

88. Schwarze MM, Hawley RG. Prevention of myeloma cell apoptosis by ectopic bcl-2 expression or interleukin 6-mediated up-regulation of bcl-xL. Cancer Res 1995; 55:22625

89. Tai WT, Shiau CW, Chen HL, Liu CY, Lin CS, Cheng AL, Chen PJ, Chen KF. Mcl-1dependent activation of Beclin 1 mediates autophagic cell death induced by sorafenib and SC-59 in hepatocellular carcinoma cells. Cell Death Dis 2013; 4:e485

90. Matsunaga K, Saitoh T, Tabata K, Omori H, Satoh T, Kurotori N, Maejima I, ShirahamaNoda K, Ichimura T, Isobe T, et al. Two Beclin 1-binding proteins, Atg14L and Rubicon, reciprocally regulate autophagy at different stages. Nat Cell Biol 2009; 11:385-96 
91. Erlich S, Mizrachy L, Segev O, Lindenboim L, Zmira O, Adi-Harel S, Hirsch JA, Stein R, Pinkas-Kramarski R. Differential interactions between Beclin 1 and Bcl-2 family members. Autophagy 2007;3:561-8

92. Yue Z, Jin S, Yang C, Levine AJ, Heintz N. Beclin1, an autophagy gene essential for early embryonic development, is a haploinsufficient tumor suppressor. Proc Natl Acad Sci U S A 2003; 100:15077-82

93. Aita VM, Liang XH, Murty VV, Pincus DL, Yu W, Cayanis E, Kalachikov S, Gilliam TC, Levine B. Cloning and genomic organization of beclin 1, a candidate tumor suppressor gene on chromosome 17q21. Genomics 1999; 59:59-65

94. Liang XH, Jackson S, Seaman M, Brown K, Kempkes B, Hibshoosh H, Levine B. Induction of autophagy and inhibition of tumorigenesis by beclin 1. Nature 1999; 402:6726

95. Qu X, Yu J, Bhagat G, Furuya N, Hibshoosh H, Troxel A, Rosen J, Eskelinen EL, Mizushima N, Ohsumi Y, et al. Promotion of tumorigenesis by heterozygous disruption of the beclin 1 autophagy gene. J Clin Invest 2003; 112:1809-20

96. Itakura E, Kishi C, Inoue K, Mizushima N. Beclin 1 forms two distinct phosphatidylinositol 3-kinase complexes with mammalian Atg14 and UVRAG. Mol Biol Cell 2008; 19:536072

97. Pattingre S, Tassa A, Qu X, Garuti R, Liang XH, Mizushima N, Packer M, Schneider MD, Levine B. Bcl-2 antiapoptotic proteins inhibit Beclin 1-dependent autophagy. Cell 2005; 122:927-39

98. Guo D, Hildebrandt IJ, Prins RM, Soto H, Mazzotta MM, Dang J, Czernin J, Shyy JY, Watson AD, Phelps M, et al. The AMPK agonist AICAR inhibits the growth of EGFRvIIIexpressing glioblastomas by inhibiting lipogenesis. Proc Natl Acad Sci U S A 2009; 106:12932-7

99. Babic I, Anderson ES, Tanaka K, Guo D, Masui K, Li B, Zhu S, Gu Y, Villa GR, Akhavan D, et al. EGFR mutation-induced alternative splicing of Max contributes to growth of glycolytic tumors in brain cancer. Cell Metab 2013; 17:1000-8

100. Rasbach KA, Schnellmann RG. Signaling of mitochondrial biogenesis following oxidant injury. J Biol Chem 2007; 282:2355-62

101. Boerner JL, Demory ML, Silva C, Parsons SJ. Phosphorylation of Y845 on the epidermal growth factor receptor mediates binding to the mitochondrial protein cytochrome $\mathrm{C}$ oxidase subunit II. Mol Cell Biol 2004; 24:7059-71

102. Demory ML, Boerner JL, Davidson R, Faust W, Miyake T, Lee I, Hüttemann M, Douglas R, Haddad G, Parsons SJ. Epidermal growth factor receptor translocation to the mitochondria: regulation and effect. J Biol Chem 2009; 284:36592-604

103. Cao X, Zhu H, Ali-Osman F, Lo HW. EGFR and EGFRvIll undergo stress- and EGFR kinase inhibitorinduced mitochondrial translocalization: a potential mechanism of EGFRdriven antagonism of apoptosis. Mol Cancer 2011; 10:26 
104. Cvrljevic AN, Akhavan D, Wu M, Martinello P, Furnari FB, Johnston AJ, Guo D, Pike L, Cavenee WK, Scott AM, et al. Activation of Src induces mitochondrial localisation of de27 EGFR (EGFRvIII) in glioma cells: implications for glucose metabolism. J Cell Sci 2011; 124:2938-50

105. Schmidt-Ullrich RK, Mikkelsen RB, Dent P, Todd DG, Valerie K, Kavanagh BD, Contessa JN, Rorrer WK, Chen PB. Radiation-induced proliferation of the human A431 squamous carcinoma cells is dependent on EGFR tyrosine phosphorylation. Oncogene 1997;

15:1191-7

106. Dittmann K, Mayer C, Fehrenbacher B, Schaller M, Kehlbach R, Rodemann HP. Nuclear EGFR shuttling induced by ionizing radiation is regulated by phosphorylation at residue Thr654. FEBS Lett 2010; 584:3878-84

107. Harris AL. Hypoxia--a key regulatory factor in tumour growth. Nat Rev Cancer 2002; 2:38-47;

108. Rouschop KM, Dubois L, Schaaf MB, van den Beucken T, Lieuwes N, Keulers TG, Savelkouls KG, Bussink J, van der Kogel AJ, Koritzinsky M, et al. Deregulation of capdependent mRNA translation increases tumour radiosensitivity through reduction of the hypoxic fraction. Radiother Oncol 2011; 99:385-91

109. Rouschop KM, Dubois LJ, Keulers TG, van den Beucken T, Lambin P, Bussink J, van der Kogel AJ, Koritzinsky M, Wouters BG. PERK/elF2 $\alpha$ signaling protects therapy resistant hypoxic cells through induction of glutathione synthesis and protection against ROS. Proc Natl Acad Sci U S A 2013; 110:4622-7

110. Overgaard J. Hypoxic modification of radiotherapy in squamous cell carcinoma of the head and neck - a systematic review and meta-analysis. Radiother Oncol 2011; 100:2232

111. Bellot G, Garcia-Medina R, Gounon P, Chiche J, Roux D, Pouysségur J, Mazure NM. Hypoxiainduced autophagy is mediated through hypoxiainducible factor induction of BNIP3 and BNIP3L via their BH3 domains. Mol Cell Biol 2009; 29:2570-81

112. Klionsky DJ, Emr SD. Autophagy as a regulated pathway of cellular degradation. Science 2000; 290:1717-21

113. Zhang H, Bosch-Marce M, Shimoda LA, Tan YS, Baek JH, Wesley JB, Gonzalez FJ, Se menza GL. Mitochondrial autophagy is an HIF-1-dependent adaptive metabolic response to hypoxia. J Biol Chem 2008; 283:10892-903

114. Papandreou I, Lim AL, Laderoute K, Denko NC. Hypoxia signals autophagy in tumor cells via AMPK activity, independent of HIF-1, BNIP3, and BNIP3L. Cell Death Differ 2008; 15:1572- 81

115. Rouschop KM, Ramaekers CH, Schaaf MB, Keulers TG, Savelkouls KG, Lambin P, Koritzinsky M, Wouters BG. Autophagy is required during cycling hypoxia to lower production of reactive oxygen species. Radiother Oncol 2009; 92:411-6

116. Stegeman H, Kaanders JH, van der Kogel AJ, lida M, Wheeler DL, Span PN, Bussink J. 
Predictive value of hypoxia, proliferation and tyrosine kinase receptors for EGFR-inhibition and radiotherapy sensitivity in head and neck cancer models. Radiother Oncol 2013; 106:383-9

117. Gong C, Bauvy C, Tonelli G, Yue W, Deloménie C, Nicolas V, Zhu Y, Domergue V, MarinEsteban $\mathrm{V}$, Tharinger $\mathrm{H}$, et al. Beclin 1 and autophagy are required for the tumorigenicity of breast cancer stemlike/progenitor cells. Oncogene 2013; 32:2261-72,1-11

118. Galavotti S, Bartesaghi S, Faccenda D, Shaked-Rabi M, Sanzone S, McEvoy A, Dinsdale D, Condorelli F, Brandner S, Campanella M, et al. The autophagyassociated factors DRAM1 and p62 regulate cell migration and invasion in glioblastoma stem cells. Oncogene 2013; 32:699-712

119. Camp ER, Summy J, Bauer TW, Liu W, Gallick GE, Ellis LM. Molecular mechanisms of resistance to therapies targeting the epidermal growth factor receptor. Clin Cancer Res 2005; 11:397-405

120. Sequist LV, Lynch TJ. EGFR tyrosine kinase inhibitors in lung cancer: an evolving story. Annu Rev Med 2008; 59:429-42

121. Li YY, Lam SK, Mak JC, Zheng CY, Ho JC. Erlotinib-induced autophagy in epidermal growth factor receptor mutated non-small cell lung cancer. Lung Cancer 2013; 81:35461

122. Zou Y, Ling YH, Sironi J, Schwartz EL, Perez-Soler R, Piperdi B. The autophagy inhibitor chloroquine overcomes the innate resistance of wild-type EGFR non-small-cell lung cancer cells to erlotinib. J Thorac Oncol 2013; 8:693-702

123. Shen J, Zheng H, Ruan J, Fang W, Li A, Tian G, Niu X, Luo S, Zhao P. Autophagy inhibition induces enhanced proapoptotic effects of ZD6474 in glioblastoma. $\mathrm{Br}$ J Cancer 2013; 109:164-71 


\section{Chapter 2}

Binding of cetuximab to the EGFRvIll deletion mutant and its biological consequences in malignant glioma cells

Published in: Radiother Oncol. 2009 Sep;92(3):393-8

Jutten B, Dubois L, Li Y, Aerts H, Wouters BG, Lambin P, Theys J, Lammering $G$ 


\section{Abstract}

Background and purpose: Despite the clinical use of cetuximab, a chimeric antibody against EGFR, little is known regarding its interaction with EGFRvIII, a frequently expressed deletion mutant of EGFR. Therefore, we investigated the interaction and the functional consequences of cetuximab treatment on glioma cells stably expressing EGFRvIII.

Materials and methods: The human glioma cell line U373 genetically modified to express EGFRvIll was used to measure the binding of cetuximab and its internalization using flow cytometry and confocal microscopy. Proliferation and cell survival were analyzed by cell growth and clonogenic survival assays.

Results: Cetuximab is able to bind to EGFRvIll and causes an internalization of the receptor and decreases its expression levels. Furthermore, in contrast to EGF, cetuximab was able to activate EGFRvIII which was evidenced by multiple phosphorylation sites and its downstream signaling targets. Despite this activation, the growth rate and the radiosensitivity of the EGFRvIIIexpressing glioma cells were not modulated.

Conclusions: Cetuximab binds to EGFRvIll and leads to the initial activation, internalization and subsequent downregulation of EGFRvIII, but it does not seem to modulate the proliferation or radiosensitivity of EGFRvIII-expressing glioma cells. Thus, approaches to treat EGFRvIll-expressing glioma cells should be evaluated more carefully. 


\section{Introduction}

EGFR-targeted strategies, such as EGFR-specific tyrosine kinase inhibitors (TKIs) and monoclonal antibodies are actively being investigated, and these are showing promising results. ${ }^{1-3}$ However, recent evidence has demonstrated that certain cancer-associated mutations, including k-Ras and PTEN can result in persistent growth pathways activation despite the blockade of EGFR ${ }^{4,5}$, thereby counteracting the efficiency of these anti-EGFR-targeted treatments. Furthermore, it has also become apparent that the different strategies to inhibit EGFR (e.g. antibody versus TKI) may act differently in the same tumour ${ }^{6-8}$. Compensatory responses by other receptors, including mutant EGFR species might be one likely reason for such failures. ${ }^{8,9}$ Furthermore, the presence of naturally occurring mutants of EGFR may in part also account for the limited clinical response to some EGFR-targeted therapies. The most common variant of the EGFR, an exon 2-7 deletion mutant named EGFRvIII, has not been detected in normal tissue, but it is found in many malignancies, such as glioblastoma, non-small lung cell carcinoma, breast cancer, prostate cancer and head and neck cancer. ${ }^{10-12}$ Based on these observations, EGFRvlll should also be considered in EGFR-targeted approaches.

Next to small synthetic TKIs, monoclonal antibodies have been developed to target EGFR. ${ }^{13}$ Cetuximab, a human-mouse chimeric monoclonal antibody, is developed to target EGFR specifically. ${ }^{13,14}$ It has been designed to inhibit endogenous ligand binding to EGFR and thereby prevent the dimerization and activation of the tyrosine kinase domain. ${ }^{15}$ Cetuximab binding leads to an internalization of the receptor resulting in the downregulation of the EGFR expression on the cell. ${ }^{16}$ However, despite its frequent clinical use, little is known regarding its interaction or activity against EGFRvIII. Therefore, the present study was undertaken to investigate the interaction and the functional consequences of cetuximab treatment on malignant glioma cells stably expressing EGFRvIII. 


\section{Materials and methods}

Reagents and cell lines

Unless specified otherwise, all reagents were obtained from Sigma Chemical Co. (Sigma-Aldrich) and all electrophoresis reagentswere from BioRad (BioRad). MEM- $\alpha$ and Geneticin were obtained from Invitrogen (Invitrogen) and fetal bovine serum (FBS) was from Hyclone.

The anti-EGFR monoclonal antibody (Sc-03 mAb) was purchased from Santa Cruz (Santa Cruz Biotechnology). The anti-Tyr1173, anti-Tyr-1086, anti-Tyr1068, anti-Tyr-992 and anti-Tyr-845 EGFR phosphorylation polyclonal antibodies (pAbs), and the anti-Akt mAb, anti-ser473-phosphorylated Akt mAb and anti-phosphorylated ERK mAb were all purchased from Cell Signaling Technology (Bioke). Anti-actin mAb was purchased from MP Biomedicals (MP Biomedicals). Secondary Abs, anti-mouse HRP-conjugated and anti-rabbit HRP-conjugated, were purchased from Cell signaling Technology (Bioke). The phbAc.EGFRvIll expression plasmid was kindly provided by D. Bigner (Dept. of Pathology, Duke University, Durham, NC, USA). The U373 glioma cell line, the Chinese Hamster Ovary $(\mathrm{CHO})$ cell line and the A431 squamous carcinoma cell line were obtained from the American Type Tissue Collection (ATTC; Rockville, MD, USA). U373 cells were grown and routinely maintained in MEMa, supplemented with $10 \%$ FBS; the stable U373 cells expressing EGFRvIII were incubated in the same medium but also with $300 \mu \mathrm{g} / \mathrm{ml}$ Geneticin. The $\mathrm{CHO}$ and $\mathrm{A} 431$ cell lines were maintained in RPMI with 5\% FBS (10\% for A431). Cells were incubated at $37^{\circ} \mathrm{C}$ with $5 \% \mathrm{CO}_{2}$ and $95 \%$ air.

Flow cytometric analysis

Flow cytometric analysis was performed as described previously. ${ }^{17}$

Immunofluorescence and confocal microscopy

Cells were grown on plastic 10-cm (immunofluorescence) or 6-cm (confocal microscopy) dishes until $80 \%$ confluency and were incubated with cetuximab- 
OG (60 nM, 24 or $3 \mathrm{~h}$, respectively, $37^{\circ} \mathrm{C}$ ). Cells were washed thrice using phosphate-buffered saline (PBS), and fixed in $3 \mathrm{ml}$ methanol on ice (10 min). Propidium iodide $(1 \mu \mathrm{g} / \mathrm{ml})$ and Hoechst, containing $1 \mu \mathrm{l} / \mathrm{ml}$ RNase (15 min, $\mathrm{RT}$ ), were used as nuclear markers. Cells were washed with PBS, $\mathrm{pH} 8$, and cyto-spinned (5'500 g), mounted with Fluorescent Mounting Medium (DAKO) and analyzed using a Leica DM5000B fluorescence microscope (Leica Microsystems) or Leica TCS SPE confocal microscope.

Immunoblotting

Experiments were performed as described previously. ${ }^{18}$ Briefly, $10 \mu \mathrm{g}(20 \mu \mathrm{g}$ for phosphor-specific antibodies) of each sample was resolved on a $4-12 \%$ Criterion SDS-PAGE gel, blotted onto a Protran nitrocellulose membrane (Schleicher \& Schuell) by electrotransfer and probed overnight with antibodies as described above. Immobilized proteins were detected using SuperSignal West Pico chemiluminescent substrate (Perbio) and by exposing the blot to $\mathrm{X}$ ray film.

Cell growth assay

To determine the growth rate, $1.4 * 10^{5}$ cells were seeded in triplicate on $6-\mathrm{cm}$ dishes. The plates were incubated under normal culture conditions in a $5 \% \mathrm{CO}_{2}$ incubator for 1-6 days. After attachment, medium was supplemented with 60 $\mathrm{nM}$ cetuximab. Plates were harvested by washing thrice with PBS followed by trypsinization. Total cell numbers were enumerated using a Coulter $Z$ Series particle counter (Beckman Coulter). Doubling times were calculated from the slope of the best-fit line during the exponential phase of growth.

Radiation of cells and clonogenic survival assay

Cells were seeded in 10-cm dishes and cultured for $72 \mathrm{~h}$ in the medium with and without $60 \mathrm{nM}$ cetuximab. During irradiation, dishes were placed in a Plexiglas jig filled with water. Cells were irradiated using an MCN 225 industrial X-ray tube (Philips) operated at $225 \mathrm{kV}$ and $10 \mathrm{~mA}$ to deliver a dose of $4 \mathrm{~Gy}$ at 
a rate of $0.85 \mathrm{~Gy} / \mathrm{min}$. Cells treated with cetuximab were seeded continuously in the medium supplemented with $60 \mathrm{nM}$ cetuximab. The clonogenic survival assay was performed as described previously. ${ }^{18}$

\section{Statistics}

Statistical analysis was carried out using GraphPad Prism version 5.01 for Windows (GraphPad Software, 2007, California, USA). Unpaired Student's ttest and non-parametric Mann-WhitneyU test for small groups were used to determine the statistical significance of differences between two independent groups of variables. For all tests a $p<0.05$ was considered to be significant.

\section{Results}

Cetuximab binds to EGFRvIII and internalizes the cetuximab-EGFRvIII complex

Despite the lack of the ligand binding domain in EGFRvIII, we demonstrate that cetuximab is able to bind to EGFRvIII ${ }^{17}$ (Fig. 1). We further investigated this binding using different doses of cetuximab in a range from $5.2 \mathrm{fM}$ to $5.2 \mu \mathrm{M}$ (Fig. 1). We found that cetuximab significantly binds to EGFRvIll reaching saturation at $5.2 \mathrm{nM}$, while the parental U373 cells do not show any significant binding (Fig. 1A). The cetuximab binding was further confirmed using immunofluorescence (Fig. 1B). A membranous staining can be observed for the U373-VIII(+) cells, while no staining is detectable for the parental U373 cells (data not shown). The A431 cells, known to overexpress the EGF receptor, served as a positive control. These observations clearly demonstrate that cetuximab binds to both EGFR and EGFRvIII.

We further analyzed the localization of the cetuximab-EGFRvIII complex using confocal microscopy. Besides a membranous staining, we observed internalization of the cetuximab-receptor complex in A431 cells for EGFR and in U373-vIII(+) cells for EGFRvIll after $3 \mathrm{~h}$ of cetuximab treatment (Fig. 1C). This suggests that cetuximab not only binds to EGFRvIII and EGFR but also internalizes both these receptors. 

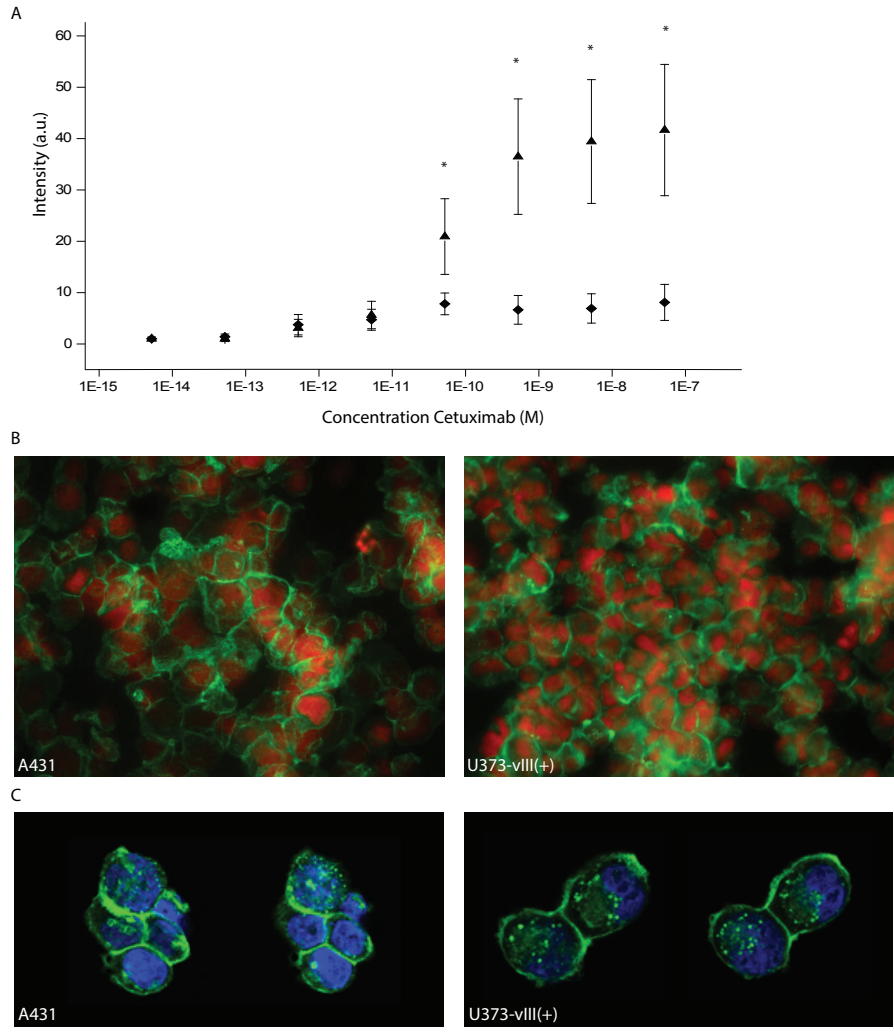

Figure 1. Cetuximab binds to and internalizes EGFRvIII. (A) Flow cytometry analysis of the binding of cetuximab to two U373 cell lines (A) U373-vIII(+) and (•) U373. Error bars indicate standard deviation of triplicate measurement, $\left(^{*}\right)$ indicates significance $p<0.05$. (B) Immunofluorescence analysis of U373-vIII(+) and A431 cells incubated for $24 \mathrm{~h}$ with cetuximab-OG. Propidium iodine was used for nuclear staining. (C) Confocal microscopy of U373-vIII(+) and A431 cells incubated for $3 \mathrm{~h}$ with cetuximab-OG. Hoechst was used for nuclear staining. Two representative slices at different depths are presented.

Cetuximab induces an initial activation of EGFRvIII

To study the biological consequences of the cetuximab binding on EGFRvIII expression, we assessed the protein expression levels of EGFRvIII under cetuximab treatment. Incubation of U373-vIII(+) cells with cetuximab in a time course revealed a slight, although not significant initial upregulation of EGFRvIII expression with a maximum at $4 \mathrm{~h}$, followed by a downregulation after 2 days 
(Fig. 2A). Interestingly, activation of EGFRvIII, as assessed by the phosphorylation at Tyr1173, strongly and significantly increased during the first hours of treatment reaching a maximum in between 1 and $4 \mathrm{~h}$ before downregulation to baseline levels at $72 \mathrm{~h}$.

In order to confirm the unresponsiveness of EGFRvIll upon ligand treatment with EGF, we incubated U373-vlll(+) and A431 cells with $3 \mathrm{ng} / \mathrm{ml}$ of EGF.

A

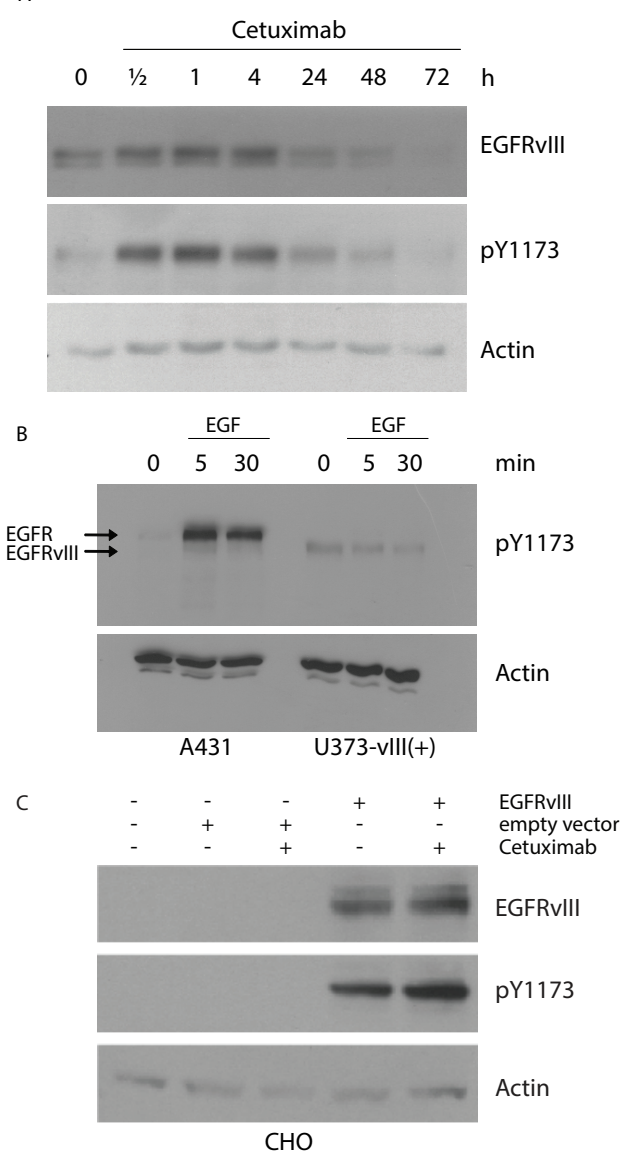

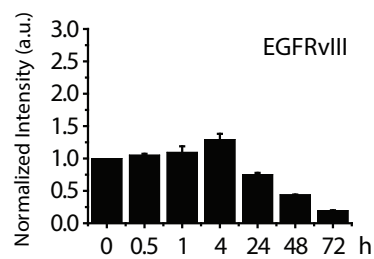

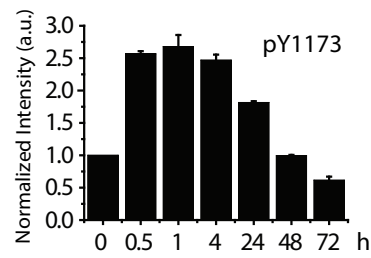

Figure 2. Cetuximab but not EGF activates EGFRvIII. (A) Western blot analysis of (phospho-) EGFRvIII expression of U373-vIII(+) cells incubated with $60 \mathrm{nM}$ cetuximab for the indicated time (h). Densitometric quantification of the band intensities is shown on the right panel normalized for actin. (B) Western blot analysis of phosphorylated EGFR in a time course after $3 \mathrm{ng} / \mathrm{ml}$ EGF treatment of A431 and U373-vIII(+) cells. (C) Western blot analysis of $\mathrm{CHO}$ cells transiently expressing EGFRvIII or an empty vector treated with cetuximab for $2.5 \mathrm{~h}$. Actin was used as a loading control. 
Western blot analysis revealed that EGFRvIll cannot be activated by EGF in U373-vIII(+) cells (Fig. 2B). Taken together, cetuximab but not EGF can initially activate EGFRvIII.

To further investigate the possible influence of other receptors expressed in U373 cells on the activation of EGFRvIll upon cetuximab treatment,we transiently overexpressed EGFRvIII in $\mathrm{CHO}$ cells, which are known to lack receptors of this family. In contrast to U373-vIII(+) cells, cetuximab did not induce an increased phosphorylation in these $\mathrm{CHO}$ cells at pY1173, indicating that the cetuximabinduced activation of EGFRvIll is cell line dependent (Fig. $2 \mathrm{C})$.

These data prompted us to further evaluate the initial activation of EGFRvIII and its downstream consequences in more detail. We investigated all known EGFRvIll phosphorylation sites and the important downstream effector kinases Erk and Akt at 30-min intervals up to $6 \mathrm{~h}$ of cetuximab treatment. Western blot

A

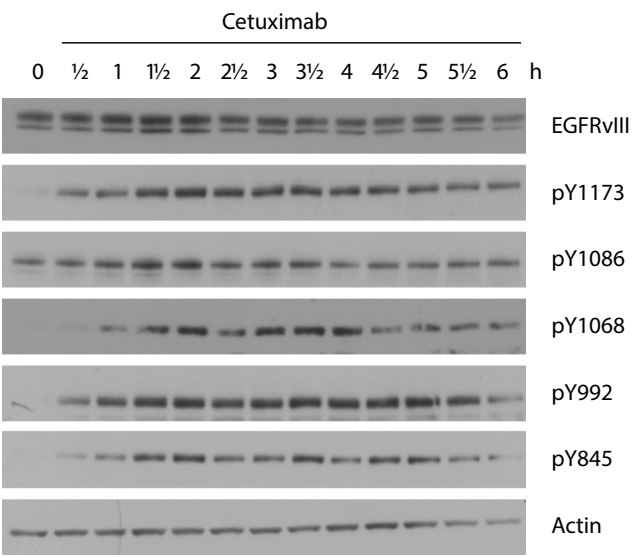

B

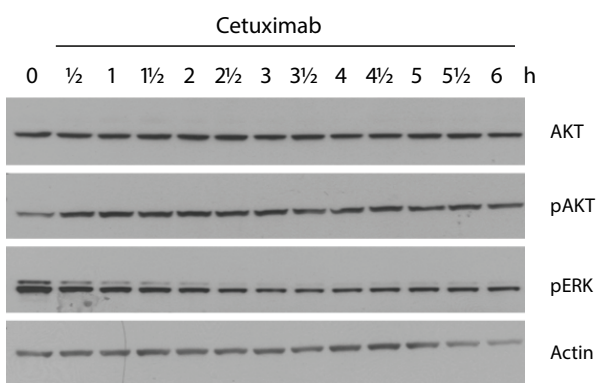

Figure 3. Phosphorylation of EGFRvIII and downstream effectors after cetuximab treatment. Western blot analysis of different EGFR phosphorylation sites (A) and Akt and Erk phosphorylation (B) for every $0.5 \mathrm{~h}$ for $6 \mathrm{~h}$ after $60 \mathrm{nM}$ cetuximab treatment. Actin was used as a loading control. 
analysis revealed that the activation of EGFRvIII resulted in phosphorylation of all tested sites (Y1173, Y1086, Y1068, Y992 and Y845). All phosphorylation sites showed a similar activation pattern with an increase up to $2.5 \mathrm{~h}$ followed by a subsequent decrease over time (Fig. 3A).

Importantly, the downstream effectors of EGFRvIII, Akt and Erk are differentially affected by the cetuximab-induced activation of EGFRvIII. While the phosphorylation of Erk constantly decreased over time, the phosphorylation of Akt slightly increased after $30 \mathrm{~min}$, indicating a potential initial activation of Akt (Fig. 3B). These data suggest that cetuximab treatment activates EGFRvIII upon binding, and that it also initially stimulates Akt, which is known to be involved in cellular proliferation, apoptosis and DNA repair.9,19,20

A

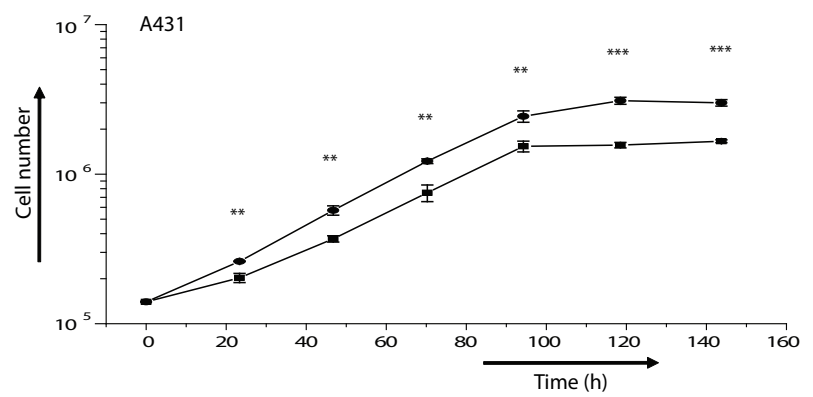

B

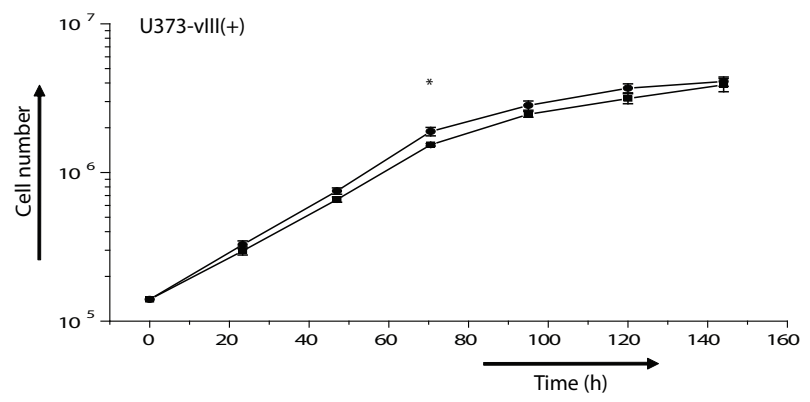

Figure 4. Cell growth upon cetuximab treatment. The influence of cetuximab on growth was evaluated by counting total numbers of A431 (A) and U373-vIII(+) (B) cells. (•) Cells incubated with $60 \mathrm{nM}$ control IgG, (घ) cells incubated with $60 \mathrm{nM}$ cetuximab. Error bars indicate standard deviation of triplicate measurement. Asterisk indicates significance $\left({ }^{*} p<0.05,{ }^{* *} p<0.01\right.$ and ${ }^{* * *} p$ $<0.001)$. 
Growth of EGFRvIll-expressing glioma cells upon cetuximab treatment

Since cetuximab has been shown to reduce the proliferation of EGFRexpressing cells, we further investigated the effect of cetuximab on the proliferation of EGFRvIll-expressing cells in vitro. We compared the growth of A431 and U373-vIII(+) cells upon incubation with cetuximab. Whereas the doubling time of A431 cells increased significantly from approximately 23 to 30 $\mathrm{h}$ upon longterm cetuximab treatment (Fig. 4A), the doubling time of U373vIII(+) cells remained unchanged at approximately $20 \mathrm{~h}$ (Fig. 4B).

Radiosensitivity of EGFRvIll-expressing glioma cells upon cetuximab treatment

Finally, to evaluate the consequences of cetuximab treatment on the radioresistant phenotype of glioma cells expressing EGFRvIII, we performed a clonogenic survival assay with the parental U373 and the U373-vIII(+) cell lines. Both cell lines were pre-treated with cetuximab for $72 \mathrm{~h}$, before the cells were irradiated with a clinically relevant single dose of $4 \mathrm{~Gy}$.

In line with earlier reports ${ }^{18,21}$, the EGFRvIII-expressing cell line U373-vIII(+) demonstrated a higher survival rate compared to the parental U373 cell line. Cetuximab treatment alone only slightly decreased the survival rate of the two

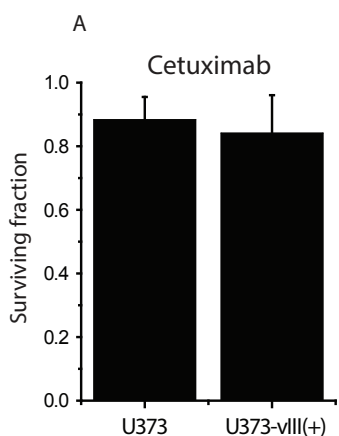

B

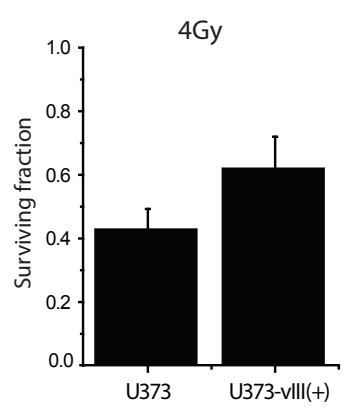

C

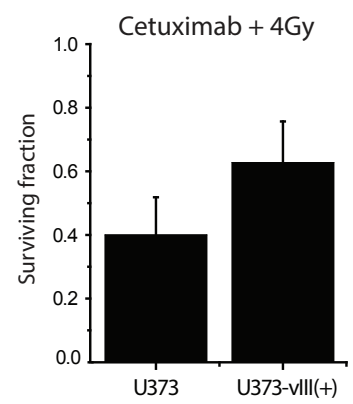

Figure 5. Clonogenic survival upon cetuximab treatment. Clonogenic survival assays were performed to determine the survival of the cell lines U373 and U373-vIII(+) $72 \mathrm{~h}$ after $60 \mathrm{nM}$ cetuximab treatment (A), $24 \mathrm{~h}$ after IR at $4 \mathrm{~Gy}(\mathrm{~B})$ and the combination of cetuximab treatment and IR at $4 \mathrm{~Gy}(\mathrm{C})$. The mean values of three independent experiments are shown. Error bars indicate standard deviation. 
tested cell lines.Importantly, the combination of cetuximab and irradiation did not alter the survival rate in both cell lines as compared with irradiation alone (Fig. 5). Thus, despite the binding and internalization of EGFRvIII upon cetuximab treatment, cetuximab did not alter the radiosensitivity of EGFRvIIIexpressing U373 cells in vitro.

\section{Discussion}

In the present report we describe the interaction of cetuximab with EGFRvIII in malignant glioma cells genetically modified to stably express EGFRvIII. We prove that in contrast to recent literature and despite the absence of the ligand binding domain, cetuximab indeed binds to EGFRvIII and initially activates and internalizes EGFRvIII before subsequent downregulation. ${ }^{3}$

To our knowledge, this is the first evidence that cetuximab not only binds to EGFRvIll but also activates it. Although binding of cetuximab to either EGFR or EGFRvIII should not lead to the activation of the receptors, this has been observed previously for the wild-type receptor. ${ }^{16,28}$ Interestingly, this activation was not observed in $\mathrm{CHO}$ cells transiently expressing EGFRvIII. This might indicate an activation mechanism related to trans-receptor activation following heterodimerization with other receptors present in $\mathrm{U} 373$ cells. $\mathrm{CHO}$ cells are characterized by a lack in ErbB receptor expression ${ }^{21}$ whereas U 373 cells also express ErbB2 and IGF-1R, both known to heterodimerize with EGFR in a variety of epithelial cancers. ${ }^{26,27}$ However, other mechanisms both upstream (integrins, integrin-growth receptor linker proteins, etc.) and downstream (e.g. caveolae related) might be involved and this might also influence the specific type of internalization of the cetuximab-EGFRvIII complex we have observed. ${ }^{29,30}$ As such, our observations can serve as a starting point to elucidate these mechanisms in more detail. Also, in contrast to EGFR, no studies have yet determined possible translocation pathways for EGFRvIll and whether they can be inhibited. ${ }^{31}$

Importantly, despite the interaction with the receptor and its eventual downregulation, cetuximab failed to reduce the growth, the survival and the displayed increased radioresistance of the U373-vIII(+) cells. ${ }^{8,18,21}$ Thus, in our model under in vitro conditions, EGFRvIll imparts a growth and survival 
advantage, which does not seem to be affected by cetuximab treatment in our performed assays. . $^{8,18,21}$ This might indicate that the current clinical approaches using cetuximab to radiosensitize malignant glioma cells, which express EGFRvIII, should be reconsidered. Indeed, other strategies targeting the phenotypical consequences of the EGFRvIll expression rather than the protein itself should be taken into consideration. These strategies could include, e.g. the inhibition of the EGFRvIll-mediated sustained downstream signaling pathways, which have also been shown to significantly affect the DNA repair machinery in cellular responses to ionizing radiation. ${ }^{4,22}$

Nevertheless, cetuximab can bind to EGFRvIII, which makes it a potential candidate for approaches to image EGFR and EGFRvIII. In this context, studies have been initiated recently. ${ }^{17,23}$ The fact that the cetuximab-EGFRvIII complex subsequently internalizes also implies that cetuximab might be potentially used in EGFR and EGFRvIll-expressing tumours as a carrier for intracellular drug delivery, e.g. approaches covalently linking monoclonal antibody fragments of cetuximab to stabilized liposomes containing chemo-therapeutic drugs or probes.$^{24,25}$

Obviously, the in vitro transfection model that was used has some limitations. Indeed, such stable in vitro overexpression might probably not resemble the native in vivo situation. Not only is the current understanding of the true role of EGFRvIII in real solid tumours limited, but the homogeneous cellular overexpression levels also largely differ from the endogenous expression levels and the distribution pattern seen in mature tumours. ${ }^{32}$ Despite this, the model used can definitely provide valuable information on the EGFRvIII-related biological mechanisms, because it enables the measurement of biological responses which would otherwise probably not have been detected at the endogenous in vivo levels due to the current detection limits.

In summary, we have implicated cetuximab as an antibody capable of binding to EGFRvIll in glioma cells with even an initial activation of the receptor. However, despite the binding and the subsequent decrease in expression, cetuximab did not modulate the cellular growth rate or the radiosensitivity of the tested cells. Our findings highlight the value of cetuximab as an imaging probe for EGFRvIII, but they also suggest that targeted approaches using cetuximab to treat malignant glioma cells expressing EGFRvIll should be 
evaluated more carefully also considering other strategies targeting the phenotypical consequences of the EGFRvIII expression rather than the protein itself. The mechanisms underlying the vlll-dependent biological changes warrant further investigation.

\section{Acknowledgements}

We wish to acknowledge financial support from the Dutch Cancer Society (KWF Grant 2006-3519 awarded to G.L., P.L. and B.W. and KWF Grant 2008-4210 awarded to P.L., J.T. and B.W.) and the Association for International Cancer research (AICR Grant 05-277 awarded to G.L., P.L. and B.W.). We further acknowledge technical assistance from Dr. Jos Broers for the confocal microscopy.

\section{References}

1. Bonner JA, Harari PM, Giralt J, et al. Radiotherapy plus cetuximab for squamous-cell carcinoma of the head and neck. N Engl J Med 2006;354:567-78.

2. Harari PM, Allen GW, Bonner JA. Biology of interactions: antiepidermal growth factor receptor agents. J Clin Oncol 2007;25:4057-65.

3. Hynes NE, Lane HA. ERBB receptors and cancer: the complexity of targeted inhibitors. Nat Rev Cancer 2005;5:341-54.

4. Jhawer M, Goel S, Wilson AJ, et al. PIK3CA mutation/PTEN expression status predicts response of colon cancer cells to the epidermal growth factor receptor inhibitor cetuximab. Cancer Res 2008;68:1953-61.

5. Lievre A, Bachet JB, Boige V, et al. KRAS mutations as an independent prognostic factor in patients with advanced colorectal cancer treated with cetuximab. J Clin Oncol 2008;26:374-9.

6. Baumann M, Krause M, Dikomey E, et al. EGFR-targeted anti-cancer drugs in radiotherapy: preclinical evaluation of mechanisms. Radiother Oncol 2007;83:238-48.

7. Krause M, Prager J, Zhou X, et al. EGFR-TK inhibition before radiotherapy reduces tumour volume but does not improve local control: differential response of cancer stem cells and nontumourigenic cells? Radiother Oncol 2007;83:316-25.

8. Lammering G. Molecular predictor and promising target: will EGFR now become a star in radiotherapy? Radiother Oncol 2005;74:89-91.

9. Lammering G, Hewit TH, Holmes M, et al. Inhibition of the type III epidermal growth factor receptor variant mutant receptor by dominant-negative EGFRCD533 enhances 
malignant glioma cell radiosensitivity. Clin Cancer Res 2004;10:6732-43.

10. Ge H, Gong X, Tang CK. Evidence of high incidence of EGFRvIll expression and coexpression with EGFR in human invasive breast cancer by laser capture microdissection and immunohistochemical analysis. Int J Cancer 2002;98:357-61.

11. Okamoto I, Kenyon LC, Emlet DR, et al. Expression of constitutively activated EGFRvIII in non-small cell lung cancer. Cancer Sci 2003;94:50-6.

12. Sok JC, Coppelli FM, Thomas SM, et al. Mutant epidermal growth factor receptor (EGFRvIII) contributes to head and neck cancer growth and resistance to EGFR targeting. Clin Cancer Res 2006;12:5064-73.

13. Goldstein NI, Prewett M, Zuklys K, Rockwell P, Mendelsohn J. Biological efficacy of a chimeric antibody to the epidermal growth factor receptor in a human tumor xenograft model. Clin Cancer Res 1995;1:1311-8.

14. Milas L, Mason K, Hunter N, et al. In vivo enhancement of tumor radioresponse by $\mathrm{C} 225$ antiepidermal growth factor receptor antibody. Clin Cancer Res 2000;6:701-8.

15. Mendelsohn J. Targeting the epidermal growth factor receptor for cancer therapy. J Clin Oncol 2002;20:1S-13S.

16. Yoshida T, Okamoto I, Okabe T, et al. Matuzumab and cetuximab activate the epidermal growth factor receptor but fail to trigger downstream signaling by Akt or Erk. Int $\mathrm{J}$ Cancer 2008;122:1530-8.

17. Aerts HJ, Dubois L, Hackeng TM, et al. Development and evaluation of a cetuximabbased imaging probe to target EGFR and EGFRvIII. Radiother Oncol 2007;83:326-32.

18. Weppler SA, Li Y, Dubois L, et al. Expression of EGFR variant vIll promotes both radiation resistance and hypoxia tolerance. Radiother Oncol 2007;83:333-9.

19. Toulany M, Baumann M, Rodemann HP. Stimulated PI3K-AKT signaling mediated through ligand or radiation-induced EGFR depends indirectly, but not directly, on constitutive K-Ras activity. Mol Cancer Res 2007;5:863-72.

20. Toulany $M$, Kehlbach $R$, Florczak $U$, et al. Targeting of AKT1 enhances radiation toxicity of human tumor cells by inhibiting DNA-PKcs-dependent DNA doublestrand break repair. Mol Cancer Ther 2008;7:1772-81.

21. Lammering G, Hewit TH, Valerie K, et al. EGFRvIll-mediated radioresistance through a strong cytoprotective response. Oncogene 2003;22:5545-53.

22. Gupta AK, Cerniglia GJ, Mick R, McKenna WG, Muschel RJ. HIV protease inhibitors block Akt signaling and radiosensitize tumor cells both in vitro and in vivo. Cancer Res 2005;65:8256-65.

23. Aerts HJ, Dubois L, Perk L, et al. Disparity between in vivo EGFR expression and 89Zrlabeled cetuximab uptake assessed with PET. J Nucl Med 2009;50:123-31.

24. Pan X, Wu G, Yang W, Barth RF, Tjarks W, Lee RJ. Synthesis of cetuximabimmunoliposomes via a cholesterol-based membrane anchor for targeting of EGFR. Bioconjug Chem 2007;18:101-8. 
25. Wu G, Barth RF, Yang W, Kawabata S, Zhang L, Green-Church K. Targeted delivery of methotrexate to epidermal growth factor receptor-positive brain tumors by means of ce tuximab (IMC-C225) dendrimer bioconjugates. Mol Cancer Ther 2006;5:52-9.

26. Morgillo F, Woo JK, Kim ES, Hong WK, Lee HY. Heterodimerization of insulinlike growth factor receptor/epidermal growth factor receptor and induction of survivin expression counteract the antitumor action of erlotinib. Cancer Res 2006;66:10100-11.

27. Schmidt-Ullrich RK, Contessa JN, Lammering G, Amorino G, Lin PS. ERBB receptor tyrosine kinases and cellular radiation responses. Oncogene 2003;22:5855-65.

28. Mandic R, Rodgarkia-Dara CJ, Zhu L, et al. Treatment of HNSCC cell lines with the EGFR-specific inhibitor cetuximab (Erbitux) results in paradox phosphorylation of tyrosine 1173 in the receptor. FEBS Lett 2006;580:4793-800.

29. Ning Y, Zeineldin R, Liu Y, Rosenberg M, Stack MS, Hudson LG. Downregulation of integrin a2 surface expression by mutant epidermal growth factor receptor (EGFRvIII) induces aberrant cell spreading and focal adhesion formation. Cancer Res 2005;65:9280-6.

30. Ning Y, Buranda T, Hudson LG. Activated epidermal growth factor receptor induces integrin alpha2 internalization via caveolae/raft-dependent endocytic pathway. J Biol Chem 2007;282:6380-7.

31. Dittmann K, Mayer C, Rodemann HP. Inhibition of radiation-induced EGFR nuclear import by C225 (cetuximab) suppresses DNA-PK activity. Radiother Oncol 2005;76:157-61.

32. Wikstrand CJ, McLendon RE, Friedman AH, Bigner DD. Cell surface localization and density of the tumor-associated variant of the epidermal growth factor receptor, EGFRvIII. Cancer Res 1997;57:4130-40. 


\section{Chapter 3}

Discriminative in vivo imaging of wtEGFR and EGFRvIll using nanobody technology

In preparation

Jutten B, Khoulati, R. el, Lammering G, Lambin, P, Van Bergen en Henegouwen $\mathrm{PM}$, and Roovers RC 


\section{Abstract}

The epidermal growth factor receptor (EGFR) has been shown to be a valid cancer target for antibody-based therapy. At present, several anti-EGFR monoclonal antibodies (mAbs) have been clinically used, but with varying degrees of success. The constitutive active variant vIII (lacking exon 2-7) of the EGFR is an oncogenic mutant of the receptor that is frequently expressed in (e.g. brain-) tumours. The expression of this truncation mutant of the receptor is an important characteristic of tumours relatively resistant to anti-EGFR therapy. Clinically used antibodies (e.g. cetuximab), do not discriminate between EGFR and EGFRvIII. As most EGFR-targeting antibodies target ligand binding and the vIII mutant does not require ligand binding for activation, VIII is resistant to antibody mediated targeting inhibition. Furthermore, EGFRvIII expressing tumours are less sensitive to the tyrosine kinase inhibitor gefitinib and require different treatment. The ability to assess the 'EGFR status' in EGFR positive tumours is therefore clinically very important. The 7D12 nanobody, which we have previously shown to be a suitable tool for imaging of EGFRexpressing tumours, also recognises both variants of the receptor and cannot discriminate wtEGFR from EGFRvIII. To be able to discriminate wtEGFR from EGFRvIll expression in vivo by means of imaging, single-domain antibody fragments were developed that specifically recognise wtEGFR or EGFRvIII. These nanobodies recognised their cognate antigen in an excess of the other form of the receptor, thereby demonstrating exquisite specificity for one of the two forms of the EGFR. Single domain antibody fragments that were developed recognised either wtEGFR (but not vIII) or demonstrated preferential binding to EGFRvIII. The latter fragments did not exclusively recognise EGFRvIII, but also bound wtEGFR when present in abundance. 


\section{Introduction}

The epidermal growth factor receptor (EGFR) is a member of a family of four related receptor tyrosine kinases (RTK), named Her or cErbB1, $-2,-3$ and $-4 .{ }^{1,2}$ Its extracellular domain consists of four subdomains, two of which are involved in ligand binding and one of which is involved in homodimerisation and heterodimerisation. ${ }^{3-5}$ EGFR propagates extracellular signals from its variety of ligands to yield diverse intracellular responses. Once activated, EGFR drives proliferation, differentiation and survival.

Mutations in the EGFR are common in various types of cancer. Overexpression of the EGF receptor is seen in over $70 \%$ of all metastatic colorectal cancers ${ }^{6}$, whereas mutations in the tyrosine kinase domain are often observed in non-small cell lung cell (NSCLC) tumours. ${ }^{7}$ The truncated and constitutively active form of the EGFR, EGFRvIII or $\Delta 2-7$, is noted in approximately $50 \%$ of EGFR over-expressing brain tumours ${ }^{8}$ and approximately $21 \%$ of all head and neck squamous cell carcinomas. ${ }^{9}$ Expression of this mutant EGFRvIll form is correlated with poor prognosis and survival. ${ }^{10}, 11$ Currently, expression of EGFRvIII is not routinely tested in clinical settings. Therefore we attempted to make antibodies suitable for both in vivo and ex vivo detection of wtEGFR or EGFRvIII expression. Detection of overexpression of wtEGFR and/or EGFRvIII is crucial for the selection of the correct treatment. E.g., wtEGFR overexpressing tumours can be treated with TKIs like gefitinib, while EGFRvIII expressing tumours require higher doses, repeated dosing, and longer exposure. ${ }^{12}$

Llama single domain heavy chain only antibody fragments, or nanobodies, are suitable for both PET-imaging ${ }^{13}$ and diagnosis on cryosections. ${ }^{14}$ Nanobodies are very stable, easy to engineer, have specificity and affinities comparable to conventional antibodies and efficiently expressed on bacteriophage. Since nanobodies are relatively small, $12-15 \mathrm{kD}$, they are ideally suited for imaging. This is because of their high tumour penetrating capacity and rapid clearance, giving a high tumour to blood ratio.

Cetuximab or other commercially available antibodies directed against EGFR do not discriminate between wtEGFR and EGFRvIll. Therefore we set out to select an EGFRvIll specific nanobody from a phage-library expressing 
nanobodies derived from a llama immunized with EGFRvIII to complement the previous selected wtEGFR specific nanobody (Egb5) from Roovers et.al. (Unpublished data).

The selected wtEGFR antibody was selected to bind to domain I since domain I is for the greater part missing in EGFRvIII. For EGFRvIII detection we sought for nanobodies that specifically recognised the exon 1 and 8 fusion containing the EGFRvIII unique glycine.

\section{Materials and Methods}

\section{E.coli strain and cell lines}

The bacterial strain used was TG1: supE thi-1 $\Delta$ (lac-proAB) $\Delta$ (mcrB-hsdSM)5 (rK- mK-) [F'traD36 proAB laclqZ $\Delta M 15]$. Her14 cells are derived from NIH 3T3 fibroblasts and stably express roughly 105 copies of the human EGFR on their cell surface. ${ }^{15,16}$ The human head and neck squamous cell carcinoma (HNSCC) cell line UM-SCC-14C was developed by- and a kind gift of Dr. T.E. Carey, Ann Arbor, MI, USA (abbreviated as 14C). The glioblastoma cell line U373-MG (cat. nr. HTB-17) and the epidermoid squamous carcinoma cell line A431 (cat. nr. CRL-1555) were bought from the ATCC. All cells were cultured in Dulbecco's Modification of Eagle Medium (DMEM: Gibco, Invitrogen, Paisley, United Kingdom) containing 7.5\% (v/v) foetal calf serum and $2 \mathrm{mM}$ L-glutamine in a humidified atmosphere without antibiotics at $37^{\circ} \mathrm{C}$ under $5 \% \mathrm{CO}_{2}$. Cells were regularly tested by PCR for the presence of mycoplasma and consistently found to be mycoplasma-free.

\section{Plasmids and constructs}

The cDNA encoding the truncated (de2-7, or vIII) variant of the EGFR ${ }^{17}$ was a kind gift from Dr. Shibuya (Graduate School of Medicine, the University of Tokyo, Tokyo, Japan). The cDNA was first re-cloned in the multiple cloning site of pCDNA3.1 Zeo (+) as Kpn1 fragment. Subsequently, the cDNA was liberated using HindIII and Not1 digestion. The insert was ligated at the 3' end to EcoR1Not1 digested PMX-IRES-GFP (Roovers et. al., unpublished), sticky ends were 
blunted by Klenow DNA polymerase and the 5 ' end of the cDNA was subsequently ligated to the vector. To synthesise an immuno-fusion of the extracellular domain (ECD) of EGFRvIII, the ECD was amplified using primers vIll-sense (5'-caagtcgtttaaacgccaccatgcgaccctccgggacggccgggggc-3') and vIII anti-sense (5'-ccgtatgagatcttcaggcccattcgttggacagccttc-3'). The resulting PCR product was then digested with Pme1 and Bglll and ligated in a Pme1-BamH1 digested, in-house developed Fc fusion vector. This construct (comprising a.a. 1-374 of EGFRvIll fused to human hinge-lgG1 Fc) was then expressed and purified as has been described for wtEGFR ECD-Fc. ${ }^{18}$ Purified, recombinant EGFR domain I (a.a. 1-183) and domain I and II (a.a. 1-308) were a kind gift from Dr. K.M. Ferguson (Department of Physiology, University of Pennsylvania school of Medicine, Philadelphia, USA). The domain I protein was biotinylated using the reactive ester (+)-Biotin N-hydroxy succinimide ester (Sigma-Aldrich, St. Louis, USA) according to the manufacturer's recommendations. Excess nonreacted ester was removed by dialysis through a dialysis membrane with a cut-off of 3.5kDa. A peptide comprising the first 16 a.a. of EGFRvIll, biotinylated at the C-terminal residue ( $\mathrm{N}$ term-LEEKKGNYVVTDHGSK-Biotin) was synthesised and purified by Isogen (de Meern, the Netherlands).

The pUR8100-myc-his vector was used for the construction of the phage library and the pUR8100-flag-his vector was used for the production of soluble nanobodies.

Immunisation of Llama Glama and library construction

Llama Glama (2 animals) were immunized with cell lines (alternating HCT116vIII and U373-VIII) stably expressing EGFRvIII, as has been described for cells expressing wtEGFR. ${ }^{19,20}$ After immunization, sera from immunized animals were tested for the successful induction of a humoral immune response towards the cell line by whole cell ELISA and nanobody phage libraries were constructed as has been described. ${ }^{19,20}$

Nanobody phage selections on EGFR domain I and EGFRvIII

For selection of nanobodies recognising EGFRvIII, the purified EGFRvIII ECD- 
Fc fusion was used. An antiserum to human Fc (Dako, Glostrup, Denmark) was coated (1 in 2000 in PBS) to the wells of a Maxisorp (NUNC, Rochester, USA) plate $\left(\mathrm{o} / \mathrm{n}\right.$ at $\left.4{ }^{\circ} \mathrm{C}\right)$ and the next day, wells were washed and blocked with $4 \%$ Marvel skimmed milk powder in PBS (MPBS) before $1 \mu \mathrm{g}$ of antigen was captured (in $2 \%$ MPBS) for the first selection round. Phage (roughly $10^{10} \mathrm{cfu}$ ) were blocked with $2 \%$ MPBS and added to the captured antigen. After incubation for 1.5 hours with shaking, the Maxisorb plate was extensively washed with PBS containing $0.1 \%(\mathrm{v} / \mathrm{v})$ tween-20 and antigen-bound phage were retrieved by competitive elution ${ }^{21}$ with $5 \mu \mathrm{M}$ vlll-peptide (30min, rt). For the second round of selection, rescued phage were blocked with 2\% MPBS and added to a near confluent dish of U373vlll cells blocked with selection medium (comprising DMEM, 10\% FCS, 25mM HEPES and 2\% Marvel). For negative (counter-) selection, a 10-fold excess of with selection medium preblocked HER14 cells (overexpressing wtEGFR ${ }^{15}$ ) were added in suspension during phage selection. After incubation of 1.5 hours with shaking at $4^{\circ} \mathrm{C}$, the dish was extensively washed with PBS and antigen bound phages were retrieved by competitive elution with $5 \mu \mathrm{M}$ vlll-peptide $\left(30 \mathrm{~min}, 4^{\circ} \mathrm{C}\right)$.

\section{ELISA}

A rabbit polyclonal serum to human IgG (Dako, Glostrup, Denmark) was coated (at a 1:2000 dilution in PBS) to the wells of a Maxisorp plate overnight at 4 degrees. Next day, plates were washed three times with PBS, blocked with $2 \%$ MPBS and ECD-Fc fusion protein (of either wtEGFR or EGFRvIII) was captured (25ng per well) for an hour at rt with shaking. Plates were washed as before and either phage (roughly $10^{8}$ colony forming units: CFU) or nanobody (200nM) were added and incubated for an hour at rt. Bound phage was detected with 1:10000 diluted peroxidase-coupled monoclonal anti-fd phage antibody (Amersham/GE Healthcare, Buckinghamshire, England); staining was performed using OPD (orthophenylene diamine) as substrate. Bound $\mathrm{VHH}$ was detected using a1:1000 diluted murine antibody against the C-terminal cMycderived epitope tag (clone 9E10), 1:2500 diluted peroxidase-coupled donkey anti-mouse antibodies (Jackson Immuno-research Laboratories) and staining using OPD. Optical density was measured at $490 \mathrm{~nm}$. 
Immuno-fluorescence staining

Cells were seeded on glass slides and transfected with the respective plasmids using Fugene HD transfection reagent (Promega, Madison, USA) according to the manufacturer's recommendations. Two days later, cells were washed with PBS, fixed with $4 \%(\mathrm{w} / \mathrm{v})$ formaldehyde in PBS for 30min. at room temperature (rt) and stained with the respective nanobodies. Briefly, fixed cells were blocked with glycine (100mM in PBS) and 2\% MPBS for 10 min each and then incubated with $200 \mathrm{nM}$ of the respective nanobodies or cetuximab in $2 \%$ MPBS. Bound nanobody was detected with an in-house developed rabbit serum to nanobodies and alexa-555 coupled goat anti-rabbit antibodies (Jackson Immuno-research Laboratories, Suffolk, UK). Bound cetuximab was detected with a rabbit anti-human IgG serum (Dako, Glostrup, Denmark) and alexa-555 coupled goat anti-rabbit antibodies. Nuclei were stained with DAPI and cells were embedded in Mowiol, containing PPD. Images were taken using an Olympus AX70 microscope equipped with a Nikon CCD camera (DXM1200) microscope.

Immuno-fluorescence staining of xenografts

Nanobodies were labelled with fluoProbes594 labelling kit according manufacturers protocol (Antibodies-online, Aachen, Germany). $4 \mu \mathrm{m}$ cryosections of A431 and U373vIll xenografts were air dried for 15' and blocked with $2 \%$ BSA in PBS for 30'. Slides were stained for 2 hours with fluoProbes 594 labelled nanobodies. After 3 times 5' washing with PBS, nuclei were stained with Hoechst $\mathrm{H}$ 33342. After 1 additional wash, slides were mounted with immunomount (Shandon, Thermo Fisher, Breda, the Netherlands). Slides were analysed using a Leica TCS SPE confocal microscope (Leica, Rijswijk, the Netherlands)

Flow cytometry

$34-\mathrm{E} 5$ nanobody was added in a serial dilution $(1.2 \mu \mathrm{M}$ to $12 \mathrm{pM})$ to $3^{*} 10^{5}$ cells (A431,U373vllI, U373wt (U373 cells with wtEGFR overexpression) and U373ev 
cells) in DMEM supplemented with 10\% FCS and incubated for 1 hour on ice in a 96 well V-shape plate. Bound nanobodies were detected using anti FLAG antibody (Sigma-Aldrich, St. Louis, USA) in a 1:2000 dilution followed by an anti-mouse alexa 488 antibody (Invitrogen, Paisley, United Kingdom) for 1 hour each. Cetuximab coupled to Oregon green ${ }^{22,23}$ was used in a $60 \mathrm{nM}$ concentration as a positive control. After a short fixation in $2 \%$ formalin, cells were analysed using on a Canto II (BD, Breda, the Netherlands).

\section{Surface Plasma Resonance}

All measurements were performed on a BIACORE T100 instrument (Biacore Life sciences, Uppsala, Sweden) at $25^{\circ} \mathrm{C}$. A 1:1 mixture of 1-ethyl-3-(3dimethylaminopropyl carbodiimide) hydrochloride and $\mathrm{N}$-hydroxysuccinimide was injected at a flow rate of $10 \mu \mathrm{L} / \mathrm{min}$ for 7 minutes to activate the surface of a CM5 sensor chip flow cell. wtEGFR and EGFRvIII Fc-fusion constructs diluted in $10 \mathrm{mM}$ acetate $\mathrm{pH} 4.0$ were coupled to the activated CM5 sensor chip up to $200 \mathrm{RU}$ at a flow rate of $10 \mu \mathrm{l} / \mathrm{min}$. The flow cell was then deactivated by means of injection of $1 \mathrm{~mol} / \mathrm{L}$ ethanolamine- $\mathrm{HCl}(\mathrm{pH} 8.5 ; 10 \mu \mathrm{L} / \mathrm{min})$ for 7 minutes. The reference cell was activated and blocked for background subtraction. IMAC purified nanobodies were diluted in HBS-EP (0.01 mol/L HEPES, $0.15 \mathrm{~mol} / \mathrm{L}$ $\mathrm{NaCl}, 3 \mathrm{mmol} / \mathrm{L}$ EDTA, and $0.005 \% \mathrm{vol} / \mathrm{vol}$ surfactant P20, $\mathrm{pH} 7.4$ ) and injected at $5 \mu \mathrm{L} / \mathrm{min}$

\section{Results}

Induction of a humoral anti-EGFRvIlI response in Llama Glama

Llama Glama were immunised with human glioma cells (U373-MG) or human colorectal cancer cells (HCT-116) genetically modified to constitutively express EGFRvIII. These cell lines were genetically modified since natural expression of EGFRvIII on tumour cells is rapidly lost when these cells are cultured under normal cell culture conditions.

The induction of a humoral immune response was followed by testing sera of the animals before and after immunisation by (whole cell-) ELISA. Both animal 

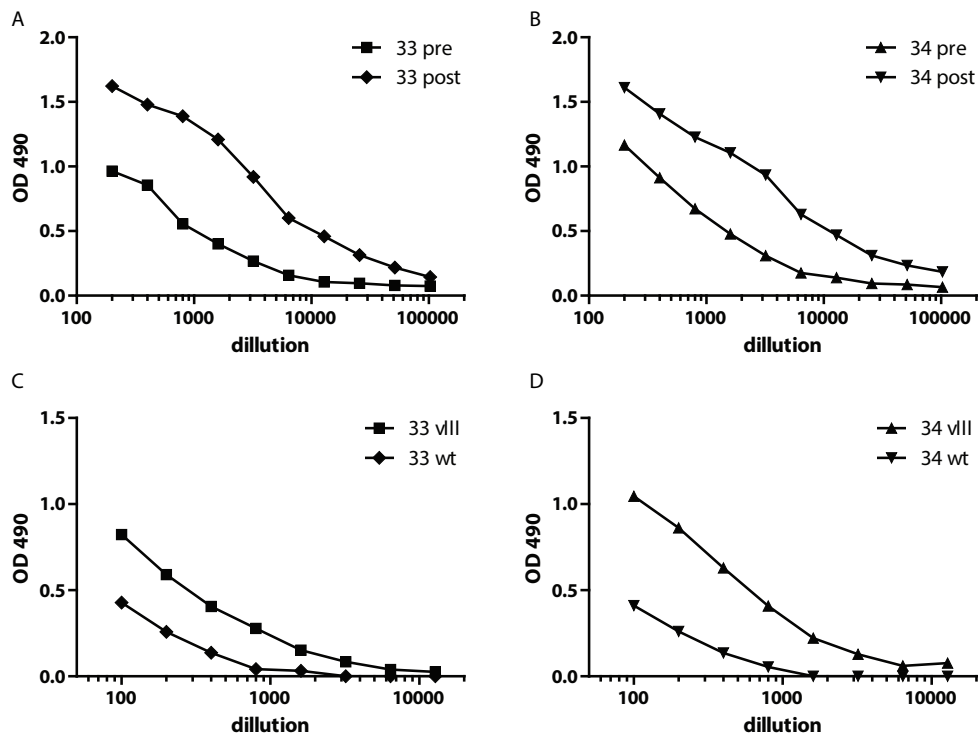

Figure 1. $(A+B)$ whole cell ELISA of pre- and post-immunization sera showing immune response towards EGFRvIII. $(C+D)$ ELISA showing reactivity of the immune sera against the extracellular domain of the EGFRvIll receptor and in a lesser extent towards the extracellular domain of wtEGFR.

33 and 34 showed an induced antibody response directed towards EGFRvIII expressing U373 cells (Fig. 1A+B). In addition, immune sera of both animals were also reactive with purified extracellular domain of the EGFRvIII but to a lesser extent with the wt extracellular domain (Fig. 1C+D). These data clearly demonstrate the successful induction of a humoral immune response towards the mutant EGF receptor.

\section{Selection and characterisation of nanobodies}

Phage nanobody repertoires were then synthesised by RT-PCR from two different lymphoid sources obtained from immunised animals 33 and 34 : peripheral blood lymphocytes (PBLs) and a biopsy from a lymph node draining the site of immunisation. This resulted in libraries of approximately 6 to $8^{*} 10^{7}$ transformants each. Direct sequencing of selected clones revealed two distinct families of nanobodies (table 1). Selected clones were retested for binding towards EGFRvIII in an ELISA using soluble nanobodies (data not shown). One 
Table 1

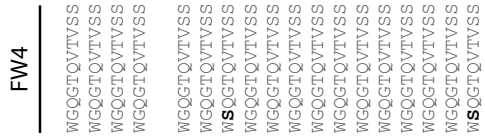

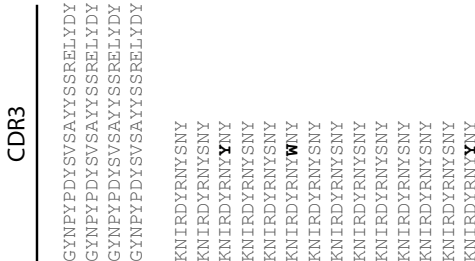

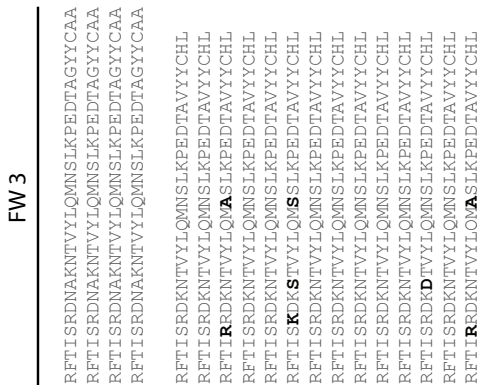

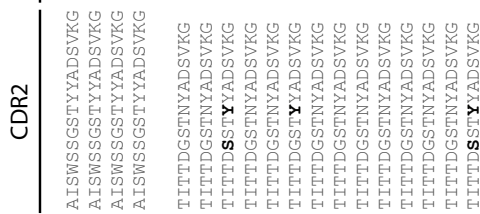

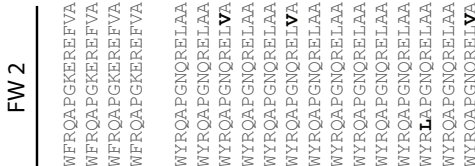

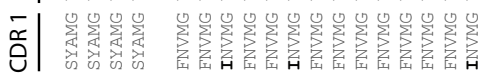

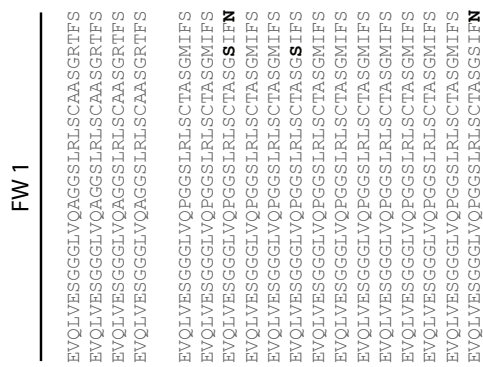

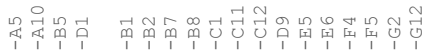

mm 

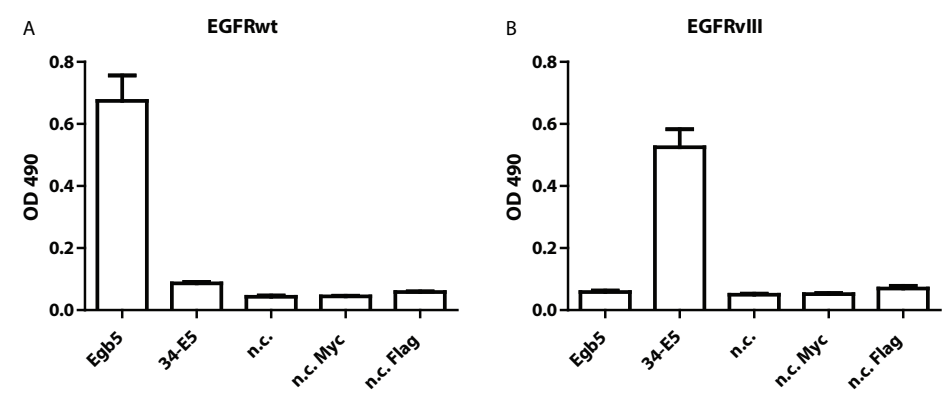

Figure 2. $(A+B)$ ELISA showing reactivity of Egb5 (wtEGFR binder) for the extracellular domain of wtEGFR and not EGFRvIII, and 34-E5 (EGFRvIII binder) showing reactivity for the extracellular domain of EGFRvIll and not wtEGFR.

nanobody, 34-E5, was retested and found strongly reactive towards EGFRvIII with negligible reactivity towards wtEGFR (Fig. 2A+B). These results suggest the successful isolation of a specific anti-EGFRvIll nanobody.

Specificity of the 34-E5 nanobody for EGFRvIll on the surface of cells was tested by mixing HER14 cells with EGFRvIll expressing U373 cells. This mixed culture was stained with rhodamine coupled egf and 34-E5 nanobody. Bound nanobody was detected using 9E10 and alexa 488 coupled anti mouse antibody. Figure 3A shows HER14 cells, with wtEGFR expression, with clear red staining and internalized egf. No binding of 34-E5 nanobody to HER14 cells is observed. EGFRvIII expressing U373-MG cells, on the other hand, show clear binding of 34-E5 nanobody with minimal binding of egf (due to low expression of wtEGFR).

To test whether the 34-E5 nanobody was capable of specifically detecting EGFRvIII in an excess of wtEGFR, HER 14 cells were transfected with a plasmid encoding EGFRvIII and GFP. Cells that express EGFRvIII (Fig. 3B, left panel) clearly show binding of 34-E5 nanobody (Fig. 3B, middle panel). This result indicates that the 34-E5 nanobody specifically binds to EGFRvIll even in the presence of wtEGFR (Fig. 3B, bottom panel).

Tumour xenografts from U373vlll and A431 cells were used to prove specificity on actual tumour sections. 34-E5 nanobody was directly labelled with fluoProbes-594. Confocal images of U373vIll xenografts showed areas with intense 34-E5 nanobody binding (Fig. 4B). In the same section unstained cell were observed, possibly stromal or other mouse tissue cells. In the A431 

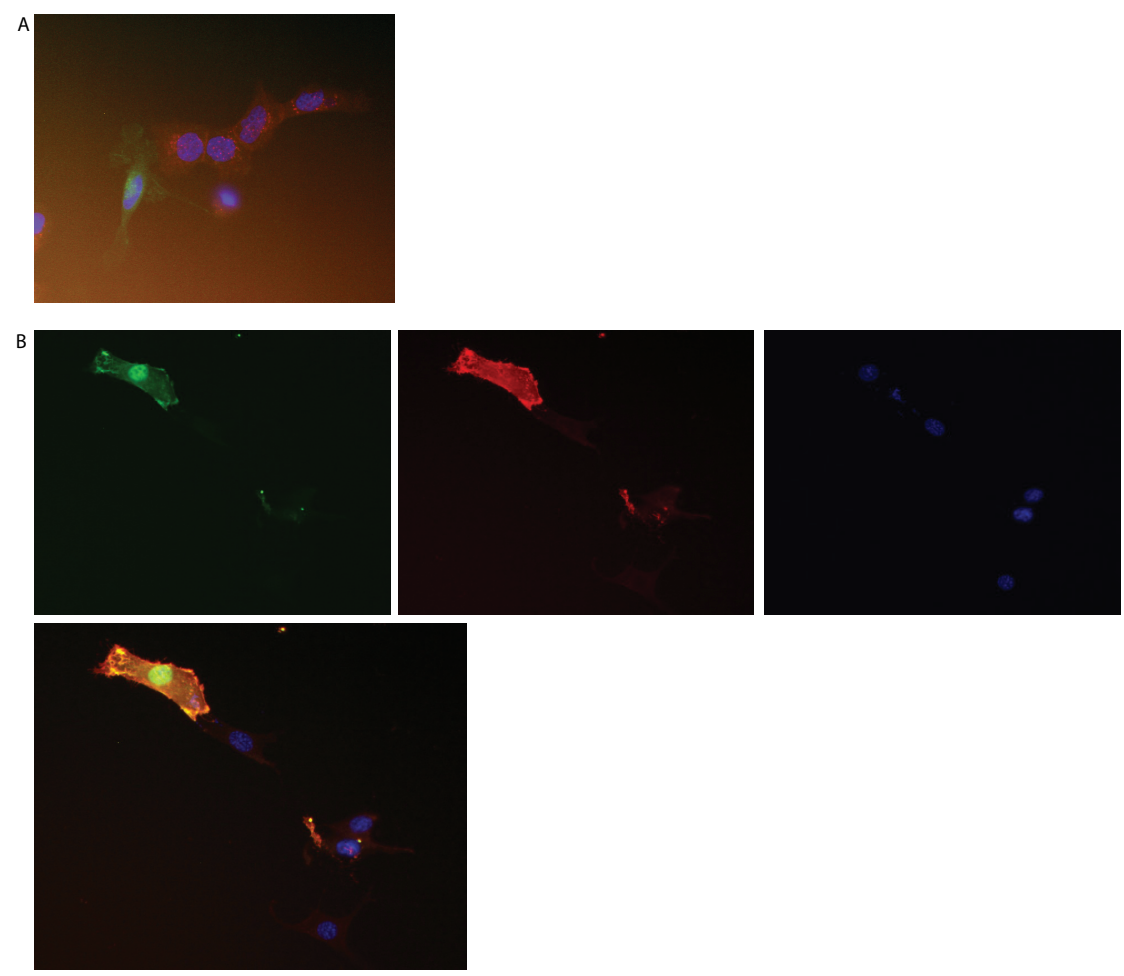

Figure 3. (A) Fluorescent immunostaining of a mix of U373vIII and HER14 cells. 34-E5 (green) binds specifically to EGFRvIll cells, whereas rhodamine coupled egf (red) binds to the HER14 cells. Nuclei in blue. (B) Fluorescent immunostaining of HER14 trasnfected with EGFRvIII and GFP. Only cells expressing EGFRvIII (green cells) are recognized by 34-E5 (in red), nuclei in blue. Overlay in bottom panel.

xenografts only marginal staining was observed in areas with cells abnormal nuclei, probably necrotic cells (Fig. 4A, left and middle panel). This was confirmed by an image of a clear necrotic area showing very intense staining due to aspecific binding which was not observed in the sections of the U373vIII xenograft. Aspecific binding to necrotic areas was confirmed using the R2 nanobody directed against the hapten ate-dye Reactive Red (RR6) ${ }^{24,25}$ showing the same staining in stromal and necrotic areas. These data suggest that the 34-E5 nanobody specifically binds to EGFRvIII.

To assess the exact affinity of the 34-E5 nanobody for EGFRvIII and wtEGFR, Surface Plasma Resonance (SPR) kinetic analysis was performed. 34-E5 showed a $0.74 \mathrm{nM}$ affinity for the Fc-EGFRvIII fusionconstruct coated on a CM5 

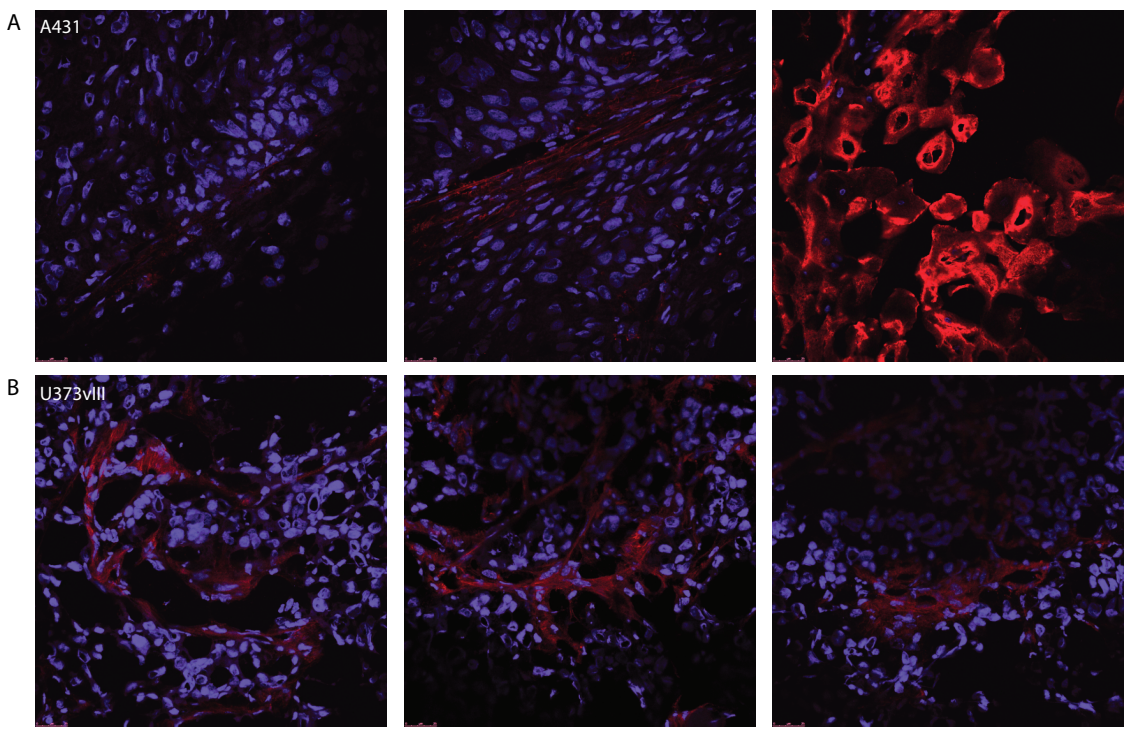

C A431 RR6
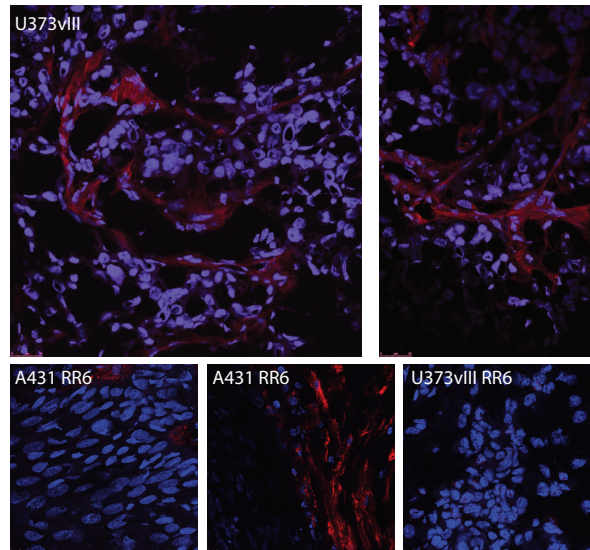

Figure 4. (A) Fluorescent immunostaining of A431 xenograft with direct labelled 34-E5. No staining was observed in the viable part of the tumour (left), a slight staining in regions with abnormal nuclei (middle, probably necrotic cells) and strong staining in clear necrotic areas (right). (B) Fluorescent immunostaining of U373vIII xenograft showing clear staining throughout the tumor. (C) Fluorescent immunostaining with RR6 for control of aspecific nanobody binding.

sensor chip (Ka 4.370*105, Kd 3.242*10-4). Unfortunately, the 34-E5 nanobody also bound to the Fc-wtEGFR fusion construct with an affinity of $1.77 \mathrm{nM}$ (Ka $3.579^{*} 10^{5}$, Kd $\left.6.335^{*} 10^{-4}\right)$. The lower affinity for wtEGFR is mostly due to its almost 2 fold higher dissociation rate constant.

To explain the observed binding of the 34-E5 nanobody to wtEGFR in the SPR and in the staining of the A431 xenografts (Fig. 4A, middle panel) an ELISA with a substantial larger amount of antigen was performed. $1 \mu \mathrm{g}$ of fusion construct was coated per well and binding of the nanobodies was tested. The control nanobody Egb5, specific for wtEGFR, only showed binding in the wells coated with wtEGFR fusion construct. The 34-E5 showed clear binding in the wells coated with EGFRvIII fusion constructs, but unfortunately also in the wells 

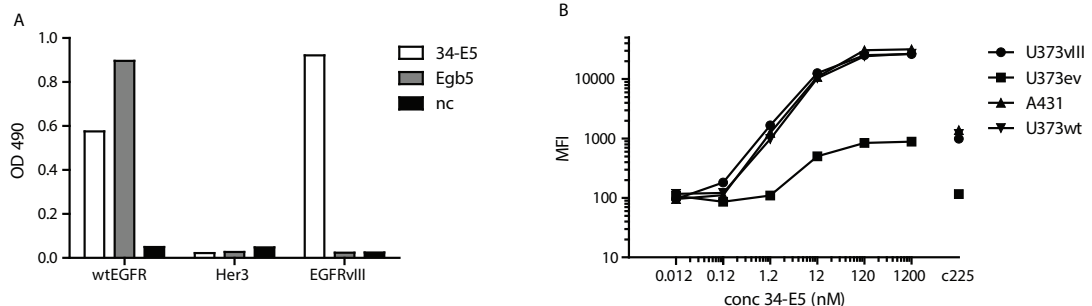

Figure 5. (A) ELISA showing specific binding of 34-E5 to EGFRvIII, but also binding to wtEGFR (B) Flow cytometry showing increased binding of 34-E5 with increased concentration of 34-E5. (U373ev is control cell line with low amounts of wtEGFR, U373wt is U373 with overexpression of wtEGFR). Cetuximab (c225) was used as a control.

coated with wtEGFR fusion construct, albeit less. Neither of the nanobodies tested showed binding to Her3, indicating that the observed binding is limited to wtEGFR/EGFRvIII (Fig. 5A).

To address the binding of 34-E5 to cells with more copies of the EGF receptor a flow cytometry analysis was performed on A431, U373vlll, U373wt (U373 cells with wtEGFR overexpression) and U373ev cells (Fig. 5B). Increasing amounts of 34-E5 nanobody showed increased binding to U373vIII cells. Unfortunately, the same was observed for A431 and U373wt cells. When concentrations of the nanobody were high enough, even binding to U373ev cells was observed. These results regrettably indicate that the selected 34-E5 nanobody is not specific for EGFRvIll when copy numbers of wtEGFR are high.

\section{Discussion}

This study set out to develop a nanobody based probe to identify EGFRvIII expression in in vitro and in vivo. Ideally this nanobody, together with the previously developed wtEGFR specific nanobody (Egb5, Roovers et al. unpublished results), could be used to discriminate between wtEGFR and EGFRvIll expressing tumours in vivo. Nanobodies were previously generated that specifically recognised domain I of the EGFR, thereby not binding to EGFRvIII. In addition, nanobodies were attempted to be selected that recognised EGFRvIll and that did not bind to wtEGFR. Although selection of the wtEGFR specific nanobody was very successful, the nanobody selected 
against EGFRvIII was only specific when low amounts of wtEGFR were present. When bigger amounts of wtEGFR, e.g. A431 cells, were presented, the EGFRvIII specific nanobody turned out not to be fully specific for EGFRvIII. This was quite unexpected since selection and screening were planned out carefully. Selections were done with depletion of wtEGFR binders by means of an access amount of HER14 cells and by selective elution with EGFRvIII peptide. During screening specificity for wtEGFR was also elaborately tested in ELISA, FACS and fluorescent-IHC. Throughout the whole screening process no indications for wtEGFR specificity were found. Unexpectedly, in the SPR experiment affinity of the EGFRvIII specific antibody to wtEGFR was detected. Indeed, when the EGFRvIII specific antibody was tested for binding to A431 cells in FACS, or higher amounts of wtEGFR were presented in ELISA, the nanobody showed binding to wtEGFR. A potential explanation could be the sensitivity of the secondary antibodies used to test nanobody binding. The used anti-FLAG antibody has a much lower detection limit then the anti-MYC 9E10. Thereby low amount of 34-E5 bound to wtEGFR could have been missed, while they were detected by the anti-FLAG antibody.

Unfortunately no real EGFRvIII specific antibody was acquired during the selection most probably due to the strong presence of the 34-E5 nanobody family. This assumption is heightened by the fact that a selection with specific elution with L8A4, a proven EGFRvIII specific mouse monoclonal ${ }^{26}$, solely resulted in 34-E5 family members. Nevertheless, we still believe a specific EGFRvIII nanobody is present in the library because of the difference in ELISA signal when post immunisation sera were tested for wtEGFR and EGFRvIII binding (Fig. 1).

Camelid derived nanobodies are able to bind to hidden antigens that are not accessible to whole antibodies, for example to the active sites of enzymes or the ligand binding domain of receptors. This property has been shown to result from their extended CDR3 loop, which is able to penetrate such sites. ${ }^{27,28}$ While the EGFRvIll peptide used in many selection and immunization ${ }^{26,29-31}$ has a type II' $\beta$-hairpin conformation, resulting in a structural conformation suitable for nanobody binding, the full length ECD of EGFRvIll on the cell surface is considered to be quite amorphous. Perhaps this makes it very difficult to find nanobody with affinity for EGFRvIII. While highly specific full length rabbit, 
mouse and human IgGs directed against EGFRvIII have been made $26,32,33$, the selection of antibody fragments like scFvs or nanobodies remain difficult. Affinity of these antibody fragments has been proven for EGFRvIII peptide or EGFRvIII on a cell surface but the required proof of absence of affinity for wtEGFR is still lacking. ${ }^{29,30,34}$

For future selections we would like to recommend more extensive depletion of wtEGFR binders. Perhaps even blocking of the epitope recognized by the 34E5 family with excessive amounts of soluble 34-E5 nanobody could be suggested, avoiding selection of the 34-E5 family. ${ }^{35}$

\section{Acknowledgements}

The authors would like to thank R. Colaris for her excellent technical assistance. R. Roovers is supported by STW grant nr. 10074.

\section{References}

1. Carpenter, G. and S. Cohen, Epidermal growth factor. J Biol Chem, 1990. 265(14): p. 7709-12.

2. Cohen, S., Nobel lecture. Epidermal growth factor. Biosci Rep, 1986. 6(12): p. 1017-28.

3. Ferguson, K.M., Structure-based view of epidermal growth factor receptor regulation. Annu Rev Biophys, 2008. 37: p. 353-73.

4. Garrett, T.P., et al., Crystal structure of a truncated epidermal growth factor receptor extracellular domain bound to transforming growth factor alpha. Cell, 2002. 110(6): p. 763-73.

5. Ogiso, H., et al., Crystal structure of the complex of human epidermal growth factor and receptor extracellular domains. Cell, 2002. 110(6): p. 775-87.

6. McKay, J.A., et al., Evaluation of the epidermal growth factor receptor (EGFR) in colorectal tumours and lymph node metastases. Eur J Cancer, 2002. 38(17): p. 2258-64.

7. Sharma, S.V., et al., Epidermal growth factor receptor mutations in lung cancer. Nat Rev Cancer, 2007. 7(3): p. 169-81.

8. Watanabe, K., et al., Overexpression of the EGF receptor and p53 mutations are mutu allyexclusive in the evolution of primary and secondary glioblastomas. Brain Pathol, 1996. 6(3):p. 217-23; discussion 23-4.

9. Smilek, P., et al., Epidermal growth factor receptor (EGFR) expression and mutations in the EGFR signaling pathway in correlation with anti-EGFR therapy in head and neck 
squamous cell carcinomas. Neoplasma, 2012. 59(5): p. 508-15.

10. Pelloski, C.E., et al., Epidermal growth factor receptor variant III status defines clinically distinct subtypes of glioblastoma. J Clin Oncol, 2007. 25(16): p. 2288-94.

11. Shinojima, N., et al., Prognostic value of epidermal growth factor receptor in patients with glioblastoma multiforme. Cancer Res, 2003. 63(20): p. 6962-70.

12. Learn, C.A., et al., Resistance to tyrosine kinase inhibition by mutant epidermal growth factor receptor variant III contributes to the neoplastic phenotype of glioblastoma multiforme. Clin Cancer Res, 2004. 10(9): p. 3216-24.

13. Vosjan, M.J., et al., Facile labelling of an anti-epidermal growth factor receptor Nanobody with 68Ga via a novel bifunctional desferal chelate for immuno-PET. Eur J Nucl Med Mol Imaging, 2011. 38(4): p. 753-63.

14. van Koningsbruggen, S., et al., Llama-derived phage display antibodies in the dissection of the human disease oculopharyngeal muscular dystrophy. J Immunol Methods, 2003. 279(1-2): p. 149-61.

15. Honegger, A.M., et al., A mutant epidermal growth factor receptor with defective protein tyrosine kinase is unable to stimulate proto-oncogene expression and DNA synthesis. Mol Cell Biol, 1987. 7(12): p. 4568-71.

16. Honegger, A.M., et al., Point mutation at the ATP binding site of EGF receptor abolishes protein-tyrosine kinase activity and alters cellular routing. Cell, 1987. 51(2): p. 199-209.

17. Yamazaki, H., et al., Amplification of the structurally and functionally altered epidermal growth factor receptor gene (c-erbB) in human brain tumors. Mol Cell Biol, 1988. 8(4): p.1816-20.

18. Roovers, R.C., et al., A biparatopic anti-EGFR nanobody efficiently inhibits solid tumour growth. Int J Cancer, 2011.

19. Roovers, R.C., et al., Efficient inhibition of EGFR signaling and of tumour growth by antagonistic anti-EFGR Nanobodies. Cancer Immunol Immunother, 2007. 56(3): p. 303317.

20. Roovers, R.C., G.A. van Dongen, and P.M. van Bergen en Henegouwen, Nanobodies in therapeutic applications. Curr Opin Mol Ther, 2007. 9(4): p. 327-35.

21. Meulemans, E.V., et al., Selection of phage-displayed antibodies specific for a cytoskeletal antigen by competitive elution with a monoclonal antibody. J Mol Biol, 1994. 244(4): p. 353-60.

22. Aerts, H.J., et al., Development and evaluation of a cetuximab-based imaging probe to target EGFR and EGFRvIlI. Radiother Oncol, 2007. 83(3): p. 326-32.

23. Jutten, B., et al., Binding of cetuximab to the EGFRvIll deletion mutant and its biological consequences in malignant glioma cells. Radiother Oncol, 2009. 92(3): p. 393-8.

24. Frenken, L.G.J., et al., Isolation of antigen specific Llama V-HH antibody fragments and their high level secretion by Saccharomyces cerevisiae. Journal of Biotechnology, 2000. 78(1): p.11-21. 
25. Spinelli, S., et al., Camelid heavy-chain variable domains provide efficient combining sites to haptens. Biochemistry, 2000. 39(6): p. 1217-1222.

26. Humphrey, P.A., et al., Anti-synthetic peptide antibody reacting at the fusion junction of deletion-mutant epidermal growth factor receptors in human glioblastoma. Proc Natl Acad Sci U S A, 1990. 87(11): p. 4207-11.

27. Desmyter, A., et al., Crystal structure of a camel single-domain $\mathrm{VH}$ antibody fragment in complex with lysozyme. Nat Struct Biol, 1996. 3(9): p. 803-11.

28. Stanfield, R.L., et al., Crystal structure of a shark single-domain antibody $V$ region in complex with lysozyme. Science, 2004. 305(5691): p. 1770-3.

29. Gupta, P., et al., Development of an EGFRvIll specific recombinant antibody. BMC Biotechnol, 2010. 10: p. 72.

30. Lorimer, I.A., et al., Recombinant immunotoxins specific for a mutant epidermal growth factor receptor: targeting with a single chain antibody variable domain isolated by phage display. Proc Natl Acad Sci U S A, 1996. 93(25): p. 14815-20.

31. Omidfar, K., et al., Production of a novel camel single-domain antibody specific for the type III mutant EGFR. Tumour Biol, 2004. 25(5-6): p. 296-305.

32. Wikstrand, C.J., et al., Cell surface localization and density of the tumor-associated vari ant of the epidermal growth factor receptor, EGFRvIll. Cancer Res, 1997. 57(18): p. 4130-40.

33. Klausz, K., et al., Complement-mediated tumor-specific cell lysis by antibody combinations targeting epidermal growth factor receptor (EGFR) and its variant III (EGFRvIII). Cancer Science, 2011. 102(10): p. 1761-8.

34. Omidfar, K., et al., Production and characterization of a new antibody specific for the mu tant EGF receptor, EGFRvIII, in Camelus bactrianus. Tumour Biol, 2004. 25(4): p. 17987.

35. Ditzel, H.J., et al., Neutralizing recombinant human antibodies to a conformational V2and CD4-binding site-sensitive epitope of HIV-1 gp120 isolated by using an epitopemasking procedure. J Immunol, 1995. 154(2): p. 893-906. 


\section{Chapter 4}

The deletion mutant EGFRvIII significantly contributes to stress resistance typical for the tumour microenvironment

Published in: Radiother Oncol. 2009 Sep;92(3):399-404

Jan Theys, Barry Jutten, Ludwig Dubois, Kasper M.A. Rouschop, Roland K. Chiu, Younan Li, Kim Paesmans, Philippe Lambin, Guido Lammering, and Bradly G. Wouters 


\section{Abstract}

Background and purpose: The epidermal growth factor receptor (EGFR) is overexpressed or mutated in many tumour types. The truncated, constitutively active EGFRvIII variant has not been detected in normal tissues but is found in many malignancies. In the current study, we have investigated the hypothesis that EGFRvIll contributes to a growth and survival advantage under tumour microenvironment-related stress conditions.

Materials and methods: U373MG doxycycline-regulated isogenic cells expressing EGFRwt or EGFRvIII were created and validated using Western blot, FACS and qRT-PCR. In vitro proliferation was evaluated with standard growth assays. Cell survival was assayed using clonogenic survival. Animal experiments were performed using NMRI-nu-xenografted mice.

Results: Inducible isogenic cell lines were created and showed high induction of EGFRwt and EGFRvIII upon doxycycline addition. Overexpression of EGFRvIll but not of EGFRwt in this model resulted in a growth and survival advantage upon different tumour microenvironment-related stress conditions in vitro. Induction of EGFRvIII increased tumour growth in vivo, which was reversible upon loss of expression.

Conclusions: Under conditions where nutrients are limited and stress is apparent, as in the tumour microenvironment, expression of EGFRvIII leads to a growth and survival advantage. These data indicate a potential selection of EGFRvIll-expressing tumour cells under such stress conditions. 


\section{Introduction}

The epidermal growth factor receptor (EGFR) is overexpressed, amplified or altered in various human epithelial tumours. ${ }^{1}$ Upon ligand binding and receptor activation, tyrosine residues in their cytoplasmatic domain are phosphorylated, resulting in the activation of downstream substrates that control cell proliferation, differentiation and survival. It is therefore not surprising that overexpression of EGFR has been shown to be associated with tumour aggressiveness and treatment resistance ${ }^{2,3}$ and that targeting of EGFR is a potentially promising treatment strategy. ${ }^{4-8}$

Several reports have documented rearrangements within the EGFR gene, especially in primary glioblastomas. ${ }^{9}$ The most common variant is EGFRvIII, which harbours an in-frame deletion resulting in a truncated receptor that lacks 267 amino acids in the extracellular binding domain. This truncation leads to important functional changes: the receptor cannot bind ligand, but is constitutively active, resulting in uncontrolled pro-oncogenic effects, thereby promoting neoplastic transformation and tumourigenicity. The EGFRvIll variant is found in many malignancies, including breast, head and neck, prostate, lung and brain tumours. In patients with glioblastoma, the EGFRvIlI mutation has an overall prevalence of $50-60 \%$ in patients whose tumours show amplification of EGFR. ${ }^{10}$ Strikingly, EGFRvIII has not been detected in normal tissues. Moreover, expression in tumour tissues in vivo is rapidly lost when cultured in vitro. ${ }^{11}$ This observation suggests that the tumour microenvironment, which is characterized by heterogeneities in nutrient supply and oxygenation, may provide suitable conditions for growth and survival of EGFRvlll-expressing tumour cells. The establishment of such a microenvironment, unlike that of any normal tissue, is a consequence of alterations in the metabolic and proliferative status of tumour cells together with a highly irregular vascular supply. ${ }^{12}$ This process is believed to occur early in the development of a tumour, and has been implicated in promoting metastasis, angiogenesis and increased malignancy. Cellular adaptive responses to this environment are critical for the continued growth of tumours. The role of EGFRvIll expression in the context of the tumour microenvironment has only been minimally defined. The present study was performed to investigate the contribution of EGFRvIll expression on 
tumour growth, as well as its importance in determining the response to microenvironmental stress.

\section{Materials and methods}

Plasmids and generation of cell lines

The glioblastoma cell line U373MG (ATCC, Manassas, VA, USA) was cultured in MEMa medium, supplemented with $10 \%$ FBS. Isogenic cell lines that can be induced to express either EGFR wildtype (wt) or EGFRvIII were generated using the Flp-In T-Rex Core Kit from Invitrogen (Breda, NL), according to the manufacturer's recommendations. These cell lines were designated U373FIpIn/ EGFRvIII and U373FIpIn/EGFRwt, respectively. To induce gene expression, doxycycline $(1 \mu \mathrm{g} / \mathrm{ml})$ was added to the medium.

Antibodies and Western blotting

Western blotting was done according to standard protocols as described..$^{13}$ Antibodies used were Sc-03 (Santa Cruz Biotechnology, Huissen, NL) for EGFRwt, L8A4 (a gift from D. Bigner, Duke University, Durham, NC, USA) for EGFRvIII and HRP-linked antirabbit (EGFRwt) or anti-mouse (EGFRvIII) secondary antibodies. Immobilized proteins were detected using ECL-plus reagent (Kirkegaard \& Perry Laboratories, NL) and by exposing blots to X-ray film.

Quantitative real-time PCR

RNA extraction was performed using the SV Total RNA Isolation System (Promega Corporation, Madison, WI, USA). cDNA was prepared using the iScript cDNA Synthesis kit (BioRad Laboratories, CA, USA). Reactions were carried out in a $25 \mu \mathrm{l}$ volume using SYBR Green Master Mix (Applied Biosystems, CA) with the ABI Prism 7700 Sequence Detection System. Values for each gene were normalized to expression levels of 18S RNA. The primer sequences used were: 
EGFRvill forward: 5'AAGAAAGGTAATTATGTGGTGACA3';

EGFRvill reverse: 5'CCGTCTTCCTCCATCTCATAGC3';

EGFRwt forward: 5'ACCTGCGTGAAGAAGTGTCC3'; and

EGFRwt reverse: 5'CCGTCTTCCTCCATCTCATAGC3'

Flow cytometric analysis

FACS analysis with Oregon Green-labeled cetuximab was performed as described..$^{14}$

In vitro growth and survival assays

For growth under control aerobic conditions, $2.5^{*} 10^{5}$ cells were seeded and incubated in $5 \% \mathrm{CO}_{2}$ for $1-7$ days. To monitor growth under stress conditions, cells were seeded and allowed to attach during overnight incubation in $5 \% \mathrm{CO}_{2}$. The following day (day 0), dishes were placed in the hypoxic chamber or medium was replaced according to the appropriate conditions. Total cell numbers were counted after trypsinization using a Coulter $Z$ series particle counter (Beckman, Mijdrecht, NL) and cell numbers normalized to the amount of cells present at day 0 . Clonogenic assays were performed as previously described..$^{13}$

Tumour xenograft model

In vivo experiments were performed using adult NMRI-nu (nu/nu) female mice. Animal facilities and experiments were in accordance with local institutional guidelines. U373 FIpIn/EGFRvIII cells were mixed with matrigel and $3^{*} 10^{6}$ cells were subcutaneously injected in the lateral flank. Induction of gene expression was done by giving animals doxycycline $(2 \mathrm{~g} / \mathrm{l})$ in their drinking water. Tumours were measured with calipers in 3 orthogonal diameters and volumes calculated with the formula $A \times B \times C \times \pi / 6$. The tumour doubling time $\left(T_{d}\right)$ was calculated using the equation $T d=\left(T-T_{0}\right) \times \log 2 /\left[\log (V)-\log \left(V_{0}\right)\right]$ where $T-T_{0}$ indicates the length of time between two measurements and $V_{0}$ and $V$ denote the tumour volume at two points of measurement. 


\section{Statistics}

Statistical analysis was carried out using the program GraphPad Prism version 5.01 for Windows (GraphPad Software, 2007, CA, USA). A Mann-Whitney U test was used to assess differences in tumour doubling times. Non-linear regression analysis was performed to compare differences between in vivo growth curves. Student's t-test was applied to determine differences in growth and survival assays.

\section{Results}

To investigate the potential influence of EGFRvIll on tumour phenotype, and to compare the effect of EGFRvIII overexpressio with that of EGFRwt, we created doxycycline-inducible U373MG glioma isogenic cell lines expressing EGFRvIII or EGFRwt. The unmodified parental U373MG cells express moderate levels of endogenous EGFRwt and have been widely recognized as a valuable in vitro and xenograft model. Previously, we constructed a Flp-In T-Rex host acceptor cell line, designated U373FIpln. Following transfection and selection, we identified clones for both EGFRvIII and EGFRwt. Quantitative PCR revealed induction of mRNA levels of $7.15 \pm 0.6$ for EGFRvIll and $7.55 \pm 0.6$ for the EGFRwt in response to doxycycline (Fig. 1A). As shown in Fig. 1B, protein levels of EGFRvill were strongly induced after exposure to doxycycline, with virtually undetectable background levels. EGFRwt is also induced in the U373FIpIn/EGFRwt cells, but to a lesser extent than EGFRvIII in the U373FIpIn/EGFRvIII cells, presumably due to the already expressed endogenous EGFRwt levels in U373FIpIn. To show that the vIll mutant and the wt receptor were properly processed and located at the cell membrane after doxycycline administration, we performed a flow cytometry experiment using Oregon Greenlabeled cetuximab (C225-OG) (Fig. 1C). Without doxycycline induction, both cell lines showed similar levels of C225-OG binding, indicating binding of C225-OG to endogenous EGFRwt receptor. Increased C225-OG binding was observed when doxycycline was added, reaching a 5- to 6-fold increase at $72 \mathrm{~h}$. Taken together,these data demonstrate that the use of this inducible system is feasible and that it results in an increase of correctly 
A.
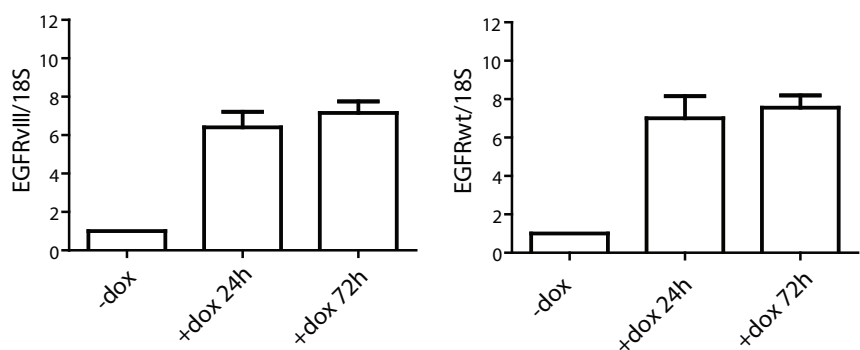

B.
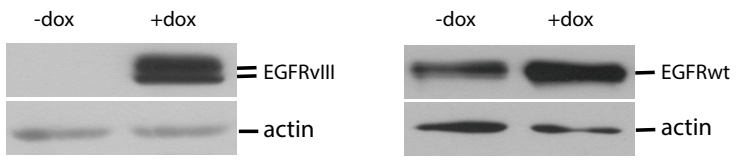

C.
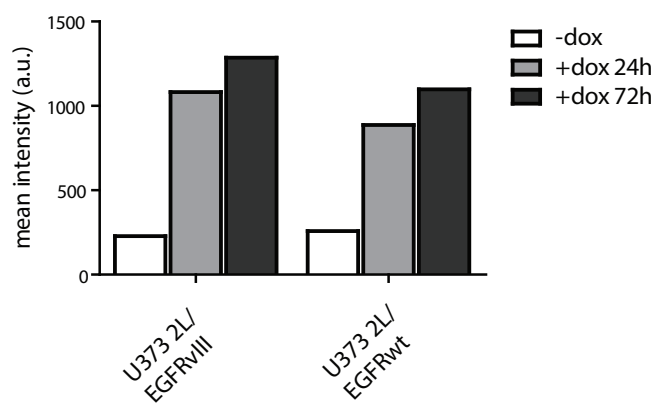

Figure 1. Validation of U373FIpIn/EGFRvIII and U373FIpIn/EGFRwt doxycycline-inducible isogenic cell line. (A) EGFRvIll and EGFRwt transcript levels in U373FIpIn transfected cells following addition of doxycycline for 24-72 h, reported as mean \pm SEM of triplicate measurements. (B) Representative Western blots showing EGFRvIII and EGFRwt protein levels after doxycycline induction for $48 \mathrm{~h}$. (C) FACS analysis of C225-OG binding to U373FIpIn/EGFRvIII and U373FIpIn/EGFRwt cells after 24-72 h induction with doxycycline.

processed receptors at the membrane of induced U373FIpln cells. To assess the biological relevance of EGFRvIll expression in relation to stress typically found in the tumour microenvironment, we evaluated the growth of U373FIpIn/EGFRvIII and U373FIpIn/ EGFRwt inducible isogenic cell lines under conditions of serum starvation, low pH, glucose deprivation, hypoxia $(0.2 \%)$ and anoxia. As shown in Fig. 2, no effect on growth after induction of EGFRvlll or EGFRwt was observed in medium containing $10 \%$ FBS. However, in conditions of serum starvation, addition of doxycycline to overexpress EGFRvIII 
A
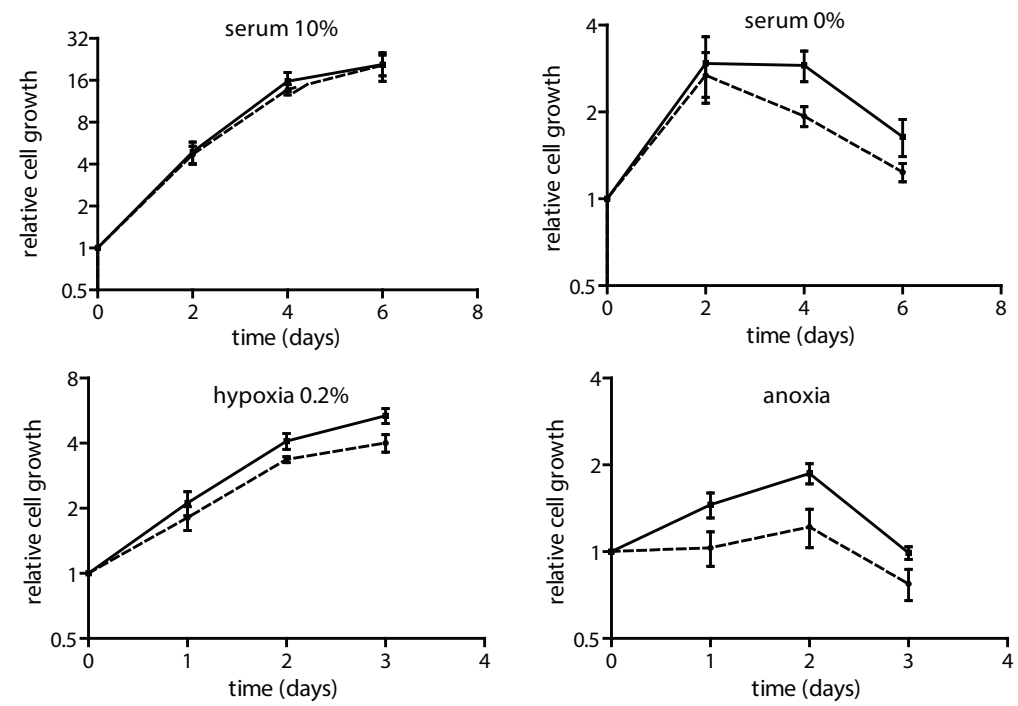

B
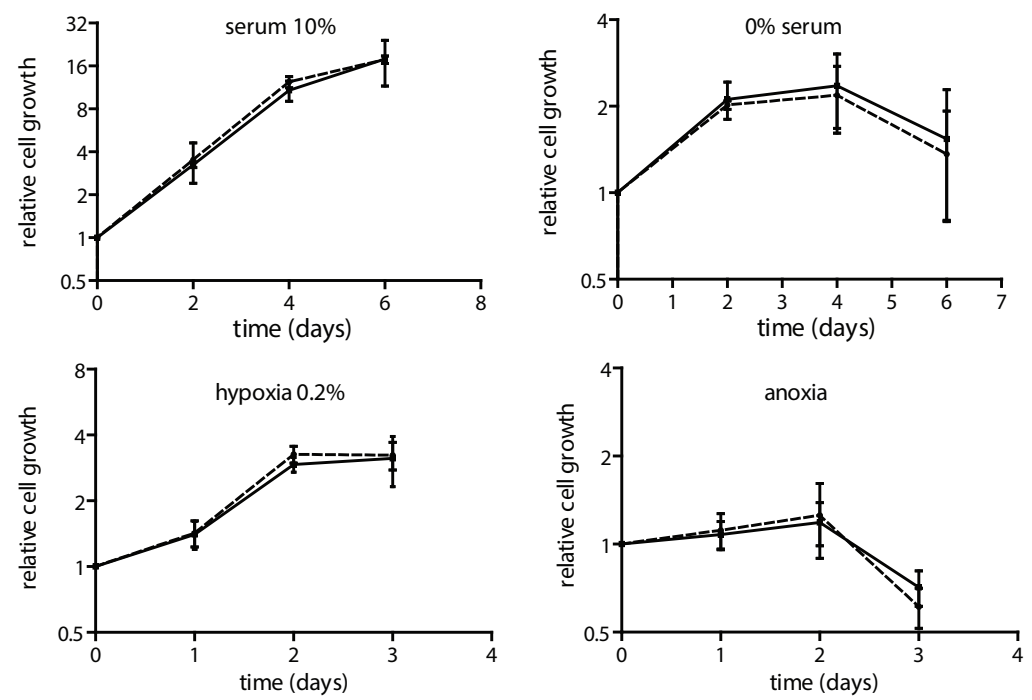

Figure 2. EGFRvIll expression results in a growth advantage in tumour microenvironment-related stress conditions. Growth was evaluated by counting cells at the indicated time points and normalized towards the number of cells present at day 0 . Experiments were done in triplicate. Data points represent the mean \pm SEM. U373FIpIn/EGFRvIII cells (A) and U373FIpIn/EGFRwt cells (B) were grown in the absence (dashed line) or presence (full line) of doxycycline at $10 \%$ or $0 \%$ serum, $0.2 \%$ hypoxia or in anoxia. 
resulted in a consistent growth advantage over non-induced cells (Fig. 2A). In contrast, no proliferation effect was detected upon induction of the EGFRwt in U373FIpIn/EGFRwt (Fig. 2B). Similarly, overexpression of EGFRvIII resulted in significant induction of proliferation in conditions of low oxygen $(0.2 \%)$ (Fig. $2 \mathrm{~A}, \mathrm{p}<0.05$ ) and complete anoxia (Fig. 2A, p<0.05 at $48 \mathrm{~h}$ ). Overexpression of EGFRwt did not lead to increased proliferation in either hypoxic or anoxic conditions (Fig. 2B), and remained at the same levels as the non-induced cells. No EGFRvIll-mediated growth promoting effect could be detected when cells were exposed to low glucose concentrations or when grown at $\mathrm{pH} 6.2$ (data not shown).
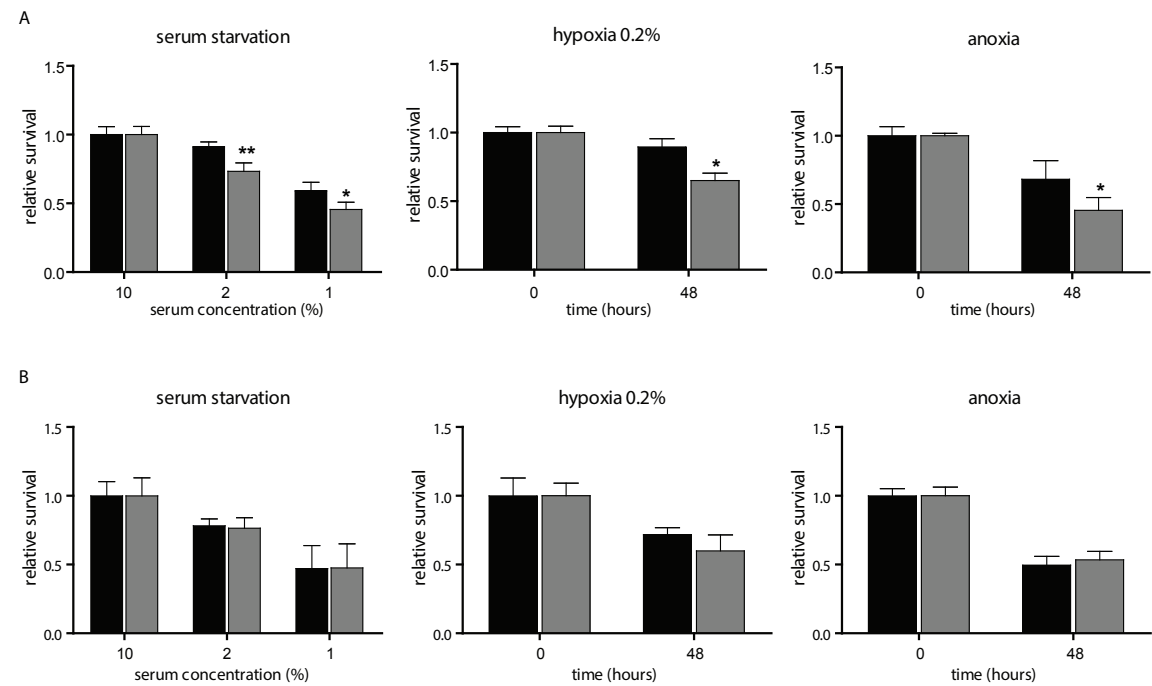

Figure 3. EGFRvIII expression influences cell survival in tumour microenvironment-related stress conditions. Clonogenic survival assay of U373FIpIn/EGFRvIII (A) and U373FIpIn/EGFRwt (B) after exposure to different concentrations of serum, to $0.2 \%$ hypoxia or to anoxia for $48 \mathrm{~h}$ with (black bars) or without (grey bars) induction with doxycycline. The mean values of three independent experiments are shown. Error bars represent SEM. $\left({ }^{*} p<0.05 ;{ }^{* *} p<0.01\right)$.

To further address the biological significance of EGFRvIll overexpression, we performed cell survival assays. In conditions with low serum concentration, EGFRvIII-expressing cells survived significantly better as cells without EGFRvIII $(p<0.01$ at $2 \%$ and $p<0.05$ at $1 \%$, Fig. 3A). We have previously demonstrated that EGFRvIII expression promotes hypoxia tolerance. ${ }^{13}$ However, those 
experiments were performed using cells that stably expressed very high levels of EGFRvill. We therefore also compared the hypoxia tolerance of the U373FIpIn/EGFRvIII and U373FIpIn/ EGFRwt cell lines following exposure to $48 \mathrm{~h}$ of hypoxia or anoxia. As shown in Fig. 3A, cells expressing EGFRvIII tolerated hypoxia $(p<0.05)$ and anoxia $(p<0.05)$ better than the cells that did not express the mutant receptor. Similar to our observations in proliferation experiments, overexpression of EGFRwt did not increase hypoxia or anoxia tolerance (Fig. 3B).

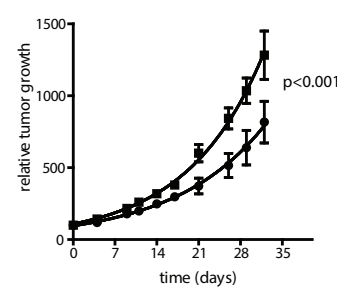

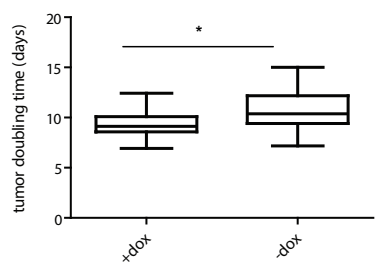

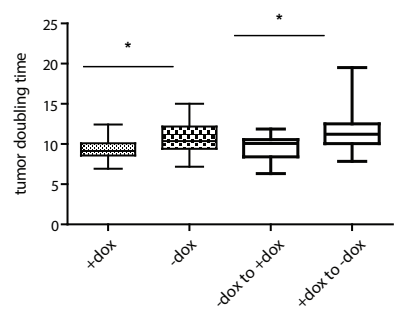

Figure 4. Induction of EGFRvIII increases tumour growth in vivo. (A) U373FIpIn/EGFRvIII xenografts were established in nude mice $(n=28)$. Mice were separated in two groups of which one group $(n=14)$ received doxycycline $(j)$ and the other group $(n=14)$ did not $(d)$. Mean tumour volumes \pm SEM are shown. Volumes were normalized to the start of the measurements (day 0 ). Data were fitted using an exponential growth equation. Non-linear regression analysis was subsequently performed to compare whether both these fits are significantly different (F-test). (B) Doubling times for tumours growing in animals that received doxycycline (left panel) or did not receive doxycycline (right panel) ( $\left.{ }^{*} p<0.05\right)$. (C) Tumour doubling times in animals after switching of animals of both groups $(n=6)$ to the other treatment group by withdrawing or administering doxycycline $\left({ }^{*} p<0.05\right)$

To investigate the influence of EGFRvIll expression on tumour growth in vivo, and to evaluate whether the observed in vitro observations could be translated to an effect in vivo, we injected U373FIpIn/EGFRvIII cells in NMRI-mice to form tumours. Mice were divided in two equal groups $(n=14)$ of which one received doxycycline. Cells in both groups formed tumours with similar efficiency and palpable tumours became visible at 12 days postinjection. As illustrated in Fig. $4 \mathrm{~A}$, the tumours in animals that were given doxycycline grew significantly $(p<$ $0.001)$ faster as those in animals without doxycycline administration, as 
evidenced by the shorter doubling time of doxycycline-induced tumours versus the non-induced xenografts (9.3 days versus 10.7 days, $p<0.05$ ) (Fig. 4B). When tumours reached volumes of $250-400 \mathrm{~mm}^{3}$, animals from both groups ( $\mathrm{n}$ $=6$ ) were randomly switched to the other treatment group by withdrawing or administering doxycycline. Intriguingly, the tumour growth rate in animals that were switched from no doxycycline to receiving doxycycline increased and their doubling time, calculated from the switch onwards, decreased (mean $T_{d}=9.5$ days) to levels similar to that of tumours in which EGFRvIII was already expressed from the start of the experiment. Conversely, tumour growth rate in animals that were taken off doxycycline decreased, and doubling times (mean $\mathrm{T}_{\mathrm{d}}=11.7$ days) became similar to those of tumours that had never been induced with doxycycline (Fig. 4C). These data indicate that EGFRvIII promotes the growth of established tumours.

\section{Discussion}

In this study, we have shown that overexpression of EGFRvIlI, but not of EGFRwt, results in a consistent proliferative and survival advantage when cells were grown under low serum, hypoxia or anoxia. Interestingly, the observed in vitro effects were also translated in increased rates of tumour growth in vivo. Previous reports have demonstrated a prominent role for EGFRvIII driving the progression of neoplastic events. ${ }^{15,16}$ However, these studies have been mainly carried out using tumour models that massively and stably overexpress the mutant receptor. To rule out clonal effects of stable transfectants and to specifically investigate the tumourigenic role of EGFRvIII, we therefore developed an isogenic inducible system. Because several different transgenes can be inserted in an identical way into the same parental cell line, it is possible to compare their contribution and effectiveness in exactly the same genetic background, overcoming a general disadvantage of models used until now. Moreover, because EGFRvIII is constitutively active, an inducible model is particularly useful for studying such a mutant. Strikingly, whereas expression of the EGFRvIII-mutant confers proliferation and survival advantages in conditions of low serum and oxygen availability, overexpression of the wt receptor did not. This might be explained by the endogenous levels of wt 
receptor that are already present on these cells (Fig. 1C), masking the effects of a further increase of receptor expression. However, other mechanisms may explain the differential phenotypic effect we observed. EGFRvIII has been shown to be increasingly associated with the adapter proteins Shc and Grb2, and increased levels of the active form of Ras. ${ }^{17} \mathrm{~A}$ recent large-scale analysis of phosphorylated proteins induced by EGFRvIll expression pointed towards PI3K signaling as the dominant pathway in glioblastoma cells. ${ }^{18}$ The significance of this pathway has also been confirmed in other studies. ${ }^{19,20}$ In a differential gene expression analysis, Ramnarain et al. revealed that expression of EGFRvIII resulted in specific upregulation of a small group of genes that are not induced by the wt receptor. These genes influence signaling pathways known to play a key role in oncogenesis and function in interconnected networks. Importantly, EGFRvIII but not EGFRwt plays an important role in generating an autocrine loop in glioma cells by increasing expression of ligands for the wt receptor ${ }^{21}$. The signaling potency of EGFRvIll might also be increased by its ability to signal for a prolonged time as a result of impaired endocytosis, inefficient ubiquitination and rapid recycling. ${ }^{22,23}$ EGFRwt has been shown to be translationally upregulated under hypoxia. ${ }^{24}$ In the stress conditions we have tested, however, this did not translate into a measurable phenotypic effect. Interestingly, we also observed an increase in EGFRvIll expression under hypoxia (unpublished observations). Although the underlying mechanism for the increased EGFRvIII levels is not yet clear, our data presented here suggest that this induction may contribute to the observed proliferative and survival advantage. In addition, this might lead to a selection of EGFRvIll-expressing cells in vivo, thereby contributing to the aggressive tumour phenotype. Interestingly, with the inducible model we have developed, tumours can be established with a parental genotype and subsequently induced to express a gene of interest to selectively examine its effect. The fact that tumours start to grow faster as soon as they are induced to express EGFRvIII (Fig. 4C), underscores the important role of EGFRvIII in the tumourigenesis process. Conversely, we also demonstrate that the increased growth rate of EGFRvIIIexpressing tumours can be reversed when the mutant receptor is no longer expressed. These data indicate that targeting EGFRvIII by silencing its expression and/or blocking its downstream effects ${ }^{25}$, even when EGFRwt 
receptor levels are relatively high as in our model, might be a promising strategy for treating cancers that contain this mutated receptor.

\section{Acknowledgements}

We acknowledge financial support from the Association for International Cancer research (AICR Grant 05-277 awarded to G.L., P.L. and B.W.), the Dutch Cancer Society (KWF Grants UM 2006-3519 awarded to G.L., P.L. and B.W., UM 2008-4068 awarded to B.W. and 2008-4210 awarded to P.L., J.T. and B.W.) and to the EU 6th and 7th framework programme (EUROXY Grants 2003502932 and METOXIA 2008-222741) awarded to P.L. and B.W.).

\section{References}

1. Herbst RS. Review of epidermal growth factor receptor biology. Int J Radiat Oncol Biol Phys 2004;59:21-6.

2. Bussink J, Kaanders JH, Van der Kogel AJ. Microenvironmental transformations by VEGF- and EGF-receptor inhibition and potential implications for responsiveness to radiotherapy. Radiother Oncol 2007;82:10-7.

3. Hynes NE, Lane HA. ERBB receptors and cancer: the complexity of targeted inhibitors. Nat Rev Cancer 2005;5:341-54.

4. Bonner JA, Harari PM, Giralt J, et al. Radiotherapy plus cetuximab for squamous-cell carcinoma of the head and neck. N Engl J Med 2006;354:567-78.

5. Baumann M, Krause M, Dikomey E, et al. EGFR-targeted anti-cancer drugs in radiotherapy: preclinical evaluation of mechanisms. Radiother Oncol 2007;83:238-48.

6. Lammering G. Molecular predictor and promising target: will EGFR nowbecome a star in radiotherapy? Radiother Oncol 2005;74:89-91.

7. Astsaturov I, Cohen RB, Harari PM. Clinical application of EGFR inhibitors in head and neck squamous cell cancer. Cancer Treat Res 2008;139:135-52.

8. Krause M, Baumann M. Clinical biomarkers of kinase activity: examples from EGFR inhibition trials. Cancer Metastasis Rev 2008;27:387-402.

9. Wong AJ, Ruppert JM, Bigner SH, et al. Structural alterations of the epidermal growth factor receptor gene in human gliomas. Proc Natl Acad Sci USA 1992;89:2965-9.

10. Gan HK, Kaye AH, Luwor RB. The EGFRvIll variant in glioblastoma multiforme. J Clin Neurosci 2009.

11. Bigner SH, Humphrey PA, Wong AJ, et al. Characterization of the epidermal growth factor receptor in human glioma cell lines and xenografts. Cancer Res 1990;50:8017-22. 
12. Wouters BG, Koritzinsky M. Hypoxia signalling through mTOR and the unfolded protein response in cancer. Nat Rev Cancer 2008;8:851-64.

13. Weppler SA, Li Y, Dubois L, et al. Expression of EGFR variant vIll promotes both radiation resistance and hypoxia tolerance. Radiother Oncol 2007;83:333-9.

14. Aerts HJ, Dubois L, Hackeng TM, et al. Development and evaluation of a cetuximabbased imaging probe to target EGFR and EGFRvIII. Radiother Oncol 2007;83:326-32.

15. Johns TG, Perera RM, Vernes SC, et al. The efficacy of epidermal growth factor receptorspecific antibodies against glioma xenografts is influenced by receptor levels, activation status, and heterodimerization. Clin Cancer Res 2007;13:1911-25.

16. Tang CK, Gong XQ, Moscatello DK, Wong AJ, Lippman ME. Epidermalgrowth factor receptor vIII enhances tumorigenicity in human breast cancer. Cancer Res 2000;60:3081-7.

17. Prigent SA, Nagane M, Lin H, et al. Enhanced tumorigenic behavior of glioblastoma cells expressing a truncated epidermal growth factor receptor is mediated through the RasShc-Grb2 pathway. J Biol Chem1996;271:25639-45.

18. Huang PH, Mukasa A, Bonavia R, et al. Quantitative analysis of EGFRvIII cellular signaling networks reveals a combinatorial therapeutic strategy for glioblastoma. Proc Natl Acad Sci USA 2007;104:12867-72.

19. Klingler-Hoffmann M, Bukczynska P, Tiganis T. Inhibition of phosphatidylinositol 3-kinase signaling negates the growth advantage imparted by a mutant epidermal growth factor receptor on human glioblastoma cells. Int J Cancer 2003;105:331-9.

20. Narita Y, Nagane M, Mishima K, Huang HJ, Furnari FB, Cavenee WK. Mutant epidermal growth factor receptor signaling down-regulates p27 through activation of the phosphatidylinositol 3-kinase/Akt pathway in glioblastomas. Cancer Res 2002;62:67649.

21. Ramnarain DB, Park S, Lee DY, et al. Differential gene expression analysis reveals generation of an autocrine loop by a mutant epidermalgrowth factor receptor in glioma cells. Cancer Res 2006;66:867-74.

22. Grandal MV, Zandi R, Pedersen MW, Willumsen BM, van Deurs B, Poulsen HS. EGFRvIII escapes down-regulation due to impaired internalization and sorting to lysosomes. Carcinogenesis 2007;28:1408-17.

23. Wang Y, Roche O, Yan MS, et al. Regulation of endocytosis via the oxygensensing pathway. Nat Med 2009;15:319-24.

24. Franovic A, Gunaratnam L, Smith K, Robert I, Patten D, Lee S. Translational upregulation of the EGFR by tumor hypoxia provides a nonmutational explanation for its overexpression in human cancer. Proc Natl Acad Sci USA 2007;104:13092-7.

25. Lammering G, Hewit TH, Holmes M, et al. Inhibition of the type III epidermal growth factor receptor variant mutant receptor by dominant-negative EGFRCD533 enhances malignant glioma cell radiosensitivity. Clin Cancer Res 2004;10:6732-43. 


\section{Chapter 5}

Autophagy inhibition abrogates the survival advantages of EGFRvIII+ cells, sensitizes tumors to irradiation and improves prognosis of GBM patients

In preparation

Jutten B, Schaaf MBE, Keulers TGH, Clarijs R, Theys J, Colaris RME, Dubois L, Savelkouls KGM, Vooijs MA, Lambin P, Bussink J, Sotelo J, Lammering GMA and Rouschop KMA 


\section{Abstract}

Expression of Epidermal growth factor receptor variant 3 (EGFRvIII), a constitutively active deletion variant of EGFR, is frequently observed in glioblastoma multiforme (GBM). We and others have shown that EGFRvIII expression is advantageous for cellular proliferation rate in vitro and in vivo. In addition, these cells display enhanced survival following radio- and chemotherapy and after exposure to metabolic stresses typically found in the tumor microenvironment such as hypoxia and starvation. Recent studies indicate that metabolically challenged cells activate autophagy to enhance their survival. In this study, we evaluated the consequence of enhanced autophagy activation in EGFRvIll expressing following exposure to starvation and hypoxia. Furthermore we assessed the therapeutic potential of targeting autophagy in EGFRvIll expressing GBM tumors.

EGFRvIll expressing cells displayed a faster and more pronounced autophagic response compared to non-EGFRvIll expressing cells during exposure to hypoxia or serum starvation. Autophagy inhibition through chloroquine (CQ) addition abrogated the enhanced proliferation and survival of EGFRvIll positive cells during stress conditions in vitro. As expected, EGFRvIll positive tumors are more radio resistant than control tumors, as displayed by decreased tumor growth delay. Interestingly, the increased radio resistance of EGFRvIII expressing tumors was abrogated by $C Q$ treatment prior to irradiation. Although effective in both GBM patient cohorts, retrospective analysis of GBM patients confirmed the enhanced effect on EGFRvIll positive GBM. (Increase in median survival for EGFRvIII 12 months versus 5 months for other).

In conclusion, EGFRvIll overexpressing cells are more dependent on autophagy for survival during metabolic stresses. Inhibition of autophagy diminished the proliferation and survival advantage of EGFRvIll overexpressing cells and sensitizes tumors to treatment. In line, although $C Q$ treatment improves survival of GBM patients, patients with EGFRvIII expressing GBM benefited most. Therefore, $C Q$ treatment could be considered as additional treatment strategy for GBM patients, and can reverse the poorer prognosis of patients with EGFRvIII positive GBM. 


\section{Introduction}

The epidermal growth factor receptor (EGFR) is a transmembrane glycoprotein and one of four members of the erbB family of tyrosine kinase receptors. Binding of one of its natural ligands results in homo- or heterodimerization of the receptor and subsequent activation of signal transduction pathways. These pathways are involved in regulating cellular proliferation, differentiation, and survival. ${ }^{1,2}$ Although present in normal cells, EGFR is overexpressed in a variety of tumors and has been associated with poor prognosis and decreased survival. ${ }^{3}$ EGFR activation also plays a role in resistance to chemotherapy and radiation treatment in tumor cells. ${ }^{4,5}$

EGFR is often mutated, leading to enhanced or sustained receptor signaling. One of the most common variant of the EGFR is an exon 2-7 deletion mutant, EGFRvIII. ${ }^{6-9}$ This deletion results in a truncated receptor that lacks 267 amino acids in the extracellular binding domain. This deletion leads to important functional changes: the receptor cannot bind its ligands, but is constitutively active, resulting in uncontrolled pro-oncogenic effects, thereby promoting neoplastic transformation and tumorigenicity. ${ }^{10-13}$ The EGFRvIII variant is found in many malignancies, including glioblastoma, non-small lung cell carcinoma, breast cancer, prostate cancer and head and neck cancer. ${ }^{6,8,9}$ More recently, EGFRvIII signaling has been implied in tumor stem cell maintenance..$^{14-16}$ In GBM patients with amplification of the EGFR gene, the overall prevalence of EGFRvIII is $50-60 \% .{ }^{17}$ Moreover, expression in tumor tissues in vivo is rapidly lost when tumor cells are subsequently cultured in vitro. ${ }^{18}$ This observation suggests that the tumor microenvironment, which is characterized by heterogeneities in nutrient supply and oxygenation, may provide suitable conditions for growth and survival of EGFRvIll-expressing tumor cells. Cellular adaptive responses to this environment are critical for the continued growth of tumors.

To survive in such environment cells will frequently upregulate autophagy. Autophagy (Greek for "self-eating") is a mechanism that protects cancer cells from low nutrient supply or therapeutic insults. ${ }^{19,20}$ It enables a cell to recycle constituents and provide itself with the necessary nutrients. Besides starvation, autophagy is upregulated during exposure to other physiological stress stimuli 
(e.g., hypoxia, energy depletion, endoplasmic reticulum stress, high temperature, and high density conditions). ${ }^{21,22}$

In initial experiments we observed that EGFRvIll expressing cells displayed increased proliferation and survival in association with increased autophagy activation when metabolically challenged. We therefore assessed the dependence of EGFRvIII expressing cells and tumors on autophagy for their increased proliferation, survival and resistance to therapy.

\section{Materials and Methods}

Reagents and cell lines

Unless specified otherwise, all reagents were obtained from Sigma Chemical Co. (the Netherlands) and all electrophoresis reagents were from Bio-Rad (the Netherlands). MEM- $\alpha$ medium was obtained from Invitrogen (Life Technologies, the Netherlands), glutamax-I (35050-038) was obtained from Life Technologies (the Netherlands), DMEM and fetal bovine serum (FBS) was from GEHealthcare (the Netherlands). The U373-MG, U-87 MG and T-47D cell lines were obtained from ATCC (USA). Cells were maintained as described by ATCC. The L8A4 antibody was kindly provided by D. Bigner (Dept. of Pathology, Duke University, Durham, NC, USA).

Western blotting

Cells were lysed and processed as described previously ${ }^{7}$ and proteins were separated on mini-PROTEAN precast gels (4-20\%, Bio-Rad). After transfer, proteins were probed with antibodies against EGFR (Santa-Cruz, USA, Sc-03), MAP1LC3b (Cell Signaling, Bioke, the Netherlands, 2775S), phosphorylated ERK (Cell signaling, 4370) and Actin (MP Biomedicals, the Netherlands, 8961001). Bound antibodies were visualized using HRP-linked anti-rabbit (Cell Signaling, 7074S) or anti-mouse (Cell Signaling, 7076S) antibodies. 
Immunofluorescence and confocal microscopy

Cells were grown on $14 \mathrm{~mm}$ round coverslips (VWR, the Netherlands) in a 24 well culture plate (Greiner Bio One, the Netherlands) until $70 \%$ confluency. The medium was replaced with either medium without $\mathrm{FBS}$, chloroquine was added in a concentration of $5 \mu \mathrm{g} / \mathrm{ml}$ and cells were incubated for 6 hours. Cells were washed 3 times with PBS and fixed in 4\% PBS-buffered paraformaldehyde (pfa) and permeabilized using $0.5 \%$ Tween-20 in PBS. Cells were probed with MAP1LC3b (Cell Signaling, 2775S) followed by anti-rabbit Alexa Fluor 488 (Invitrogen, A11006), and analyzed using a Leica TCS SPE confocal microscope.

\section{Proliferation curves}

Cells were seeded in a 24-well culture plate (Greiner Bio One) and incubated under either normal, serum starved or hypoxic conditions. Chloroquine was used at a $5 \mu \mathrm{g} / \mathrm{ml}$. Every 24 hours a plate was washed and fixed with $4 \%$ pfa. Plates were stained with $0.5 \mathrm{ml} 0.1 \%$ cristalviolet for $15^{\prime}$ at RT, after extensive washing with double distilled water cells were lysed with $0.5 \mathrm{ml} 10 \%$ acetic acid for $15^{\prime}$ at RT. Absorbance was measured at $590 \mathrm{~nm}$.

Clonogenic Survival assay

Cells were counted using a Coulter Z Series particle counter (Beckman Coulter, the Netherlands) and seeded in triplicate $6 \mathrm{~cm}$ dishes. For starvation experiments, cells were seeded for clonogenic survival prior to the starvation. After serum starvation for up to 4 days, the medium was replaced with normal medium and cells were incubated under standard culture conditions until colonies formed (10 days). For hypoxia experiments, cells were incubated at hypoxic conditions $\left(\mathrm{O}_{2}<0.02 \%\right)$ for up to 3 days. Cells, including detached cells, were harvested, seeded and incubated under standard culture conditions until colonies formed (10 days). Colonies were fixed and stained with $0.4 \%$ bromophenol blue in $70 \%$ ethanol. Plating efficiency was determined by counting colonies consisting of $\geq 50$ cells and corrected for the number of cells 
seeded.

In vivo experiments

Animal facilities and experiments were in accordance with local institutional guidelines and approved by the local animal welfare committee. Experiments were performed as described previously. ${ }^{22}$ Briefly, tumors were grown in NMRInu (nu/nu) female mice. U373-EGFRvIII and U373 control cells in matrigel were injected subcutaneously $\left(1.5 \times 10^{6}\right.$ cells). Tumor size was assessed by caliper measurement in 3 orthogonal diameters. Mice treated with $C Q$ received 60 $\mathrm{mg} / \mathrm{kg} \mathrm{CQ}$ for 7 consecutive days via intraperitoneal injection. Tumors were positioned in the irradiation field using a custom-built jig and irradiated with a single dose of $10 \mathrm{~Gy}$ (15 MeV e-) using a linear accelerator (Varian). ${ }^{23}$

Immunohistochemistry and image processing

Frozen, acetone-fixed sections were stained by using anti-pimonidazole (Chemicon, Merck Millipore, Germany), anti LC3b (Abgent, USA, AP1806a) and anti EGFR (Santa Cruz, sc-03). For quantitative analysis, the slides were scanned by a computerized digital image processing system by using a highresolution intensified solid-state camera on a fluorescence microscope (Axioskop; Zeiss, the Netherlands) with a computer-controlled motorized stepping stage. Tumor necrosis was evaluated, relative to the total tumor area, morphologically by using H\&E staining. Tumor hypoxic fraction and vascular density (structures per square millimeter) were determined relative to the viable tumor tissue (necrosis excluded).

\section{Results}

EGFRvIll cells depend more on autophagy during starvation in vitro

EGFR is often overexpressed or mutated in glioblastoma. One of the most commonly found mutation is the constitutive active deletion variant EGFRvIII. 
a

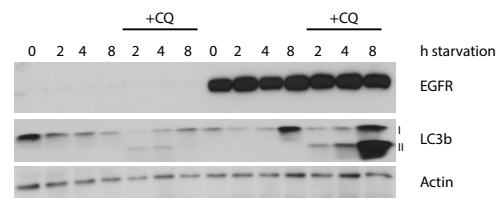

b
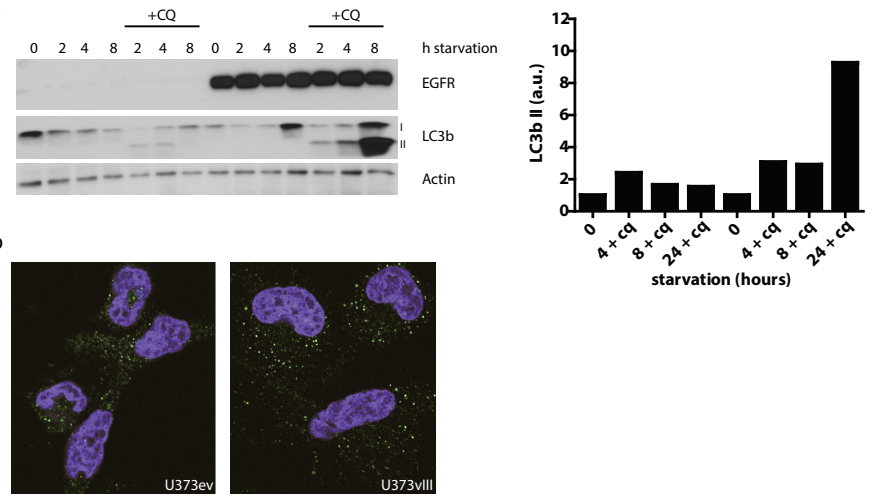

C
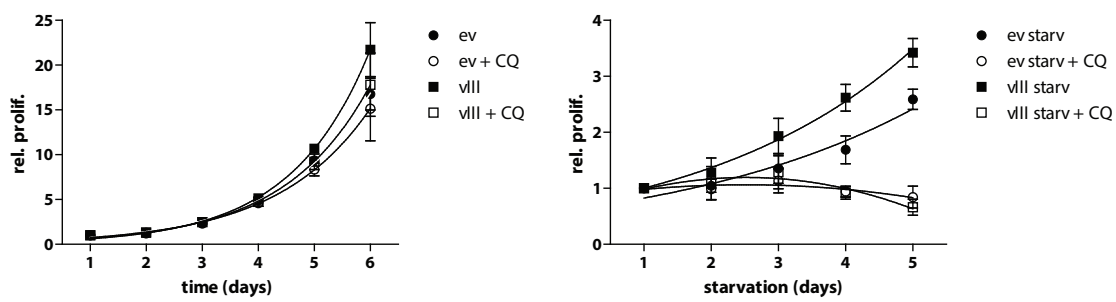

d

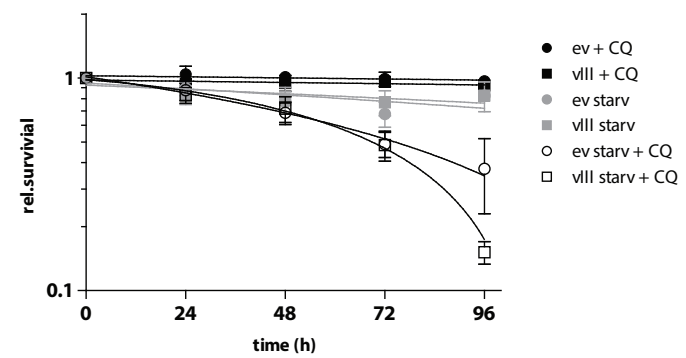

Figure 1. (A) EGFRvIII expressing glioma cells display increased autophagy under starvation. (B) EGFRvIll expressing cells display more autophagosomes under starvation (nuclei in blue, LC3b in green). (C) Proliferation assay of EGFRvIII and control cells under normal growth conditions, CQ was used at a non-toxic concentration $(5 \mu \mathrm{g} / \mathrm{ml}$ ) (left panel). EGFRvlll cells proliferate faster under starvation but this can be abrogated by inhibition of autophagy (right panel). (D) EGFRvIll survive equally well under starvation as control cells but are more sensitive for autophagy inhibition under this condition.

The tumor microenvironment is characterized by the presence of regions that are low in oxygen (hypoxia) and nutrient supply. Previously we and others have shown that cells deprived of nutrients or oxygen rapidly activate autophagy. ${ }^{22}$ 
Considering the high proliferation rate and nutritional demand of EGFRvIII cells ${ }^{24}$, we explored autophagic flux in EGFRvill cells. To determine autophagy activity, cells were exposed to nutrient deprivation in the absence and presence of chloroquine (CQ). Assessment of LC3b-II accumulation allows determination of autophagic flux. ${ }^{25,26}$ Indeed, when malignant glioma U 373 cells were deprived from serum we observed a faster and more pronounced induction of autophagy in the EGFRvIll expressing cells (Fig. 1A left panel, right panel is a quantification of LC3b-II). We observed similar effects in another glioma line U-87 MG (fig S1) and in a prostate cancer line T-47D (data not shown). In accordance, more and larger autophagosomes were observed in the EGFRvIII expressing cells compared to the control cells (Fig. 1B). To determine if the higher autophagic flux contributed to survival advantages of EGFRvIll expressing cells, we assessed proliferation during normal and starvation culture conditions. As described previously ${ }^{12}$, during normal culture conditions, EGFRvIll expressing cells proliferate faster. Addition of chloroquine had no significant effect on proliferation of either control or EGFRvIll expressing cells (Fig. 1C), indicating that the used concentration is non-toxic at this dose. As expected, we observed that EGFRvIII expressing cells proliferate faster during serum starvation conditions compared to control cells (Fig. 1C) and this could be abrogated by the addition of chloroquine $(\mathrm{CQ})$ to the medium. To assess whether targeting autophagy decreases survival of EGFRvIll expressing cells during metabolic stress, survival was assessed by clonogenic survival. During normal culture conditions, the chosen $\mathrm{CQ}$ dose proved nontoxic, and also serum starvation did not significantly alter clonogenic survival (Fig. 1D). However, when combining starvation with addition of CQ more EGFRvIll cells were killed. Taken together, these data suggest a more pronounced autophagic response and a higher dependency of autophagy in EGFRvIll expressing cells in vitro.

EGFRvIll cells depend more on autophagy during starvation in vitro

To assess if the enhanced autophagic response was restricted to nutrient deprivation, we assessed the autophagic response and dependency during hypoxia exposure, another stress typically found in the tumor microenvironment. Again, EGFRvIll cells displayed an enhanced autophagic 
a

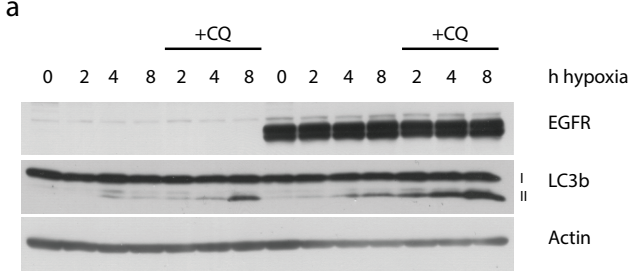

b

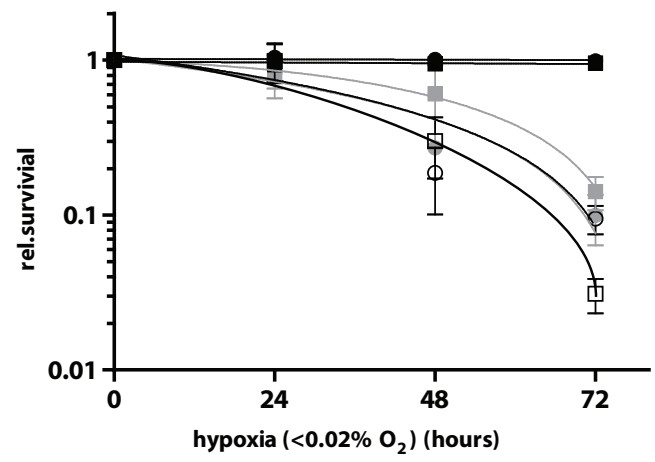

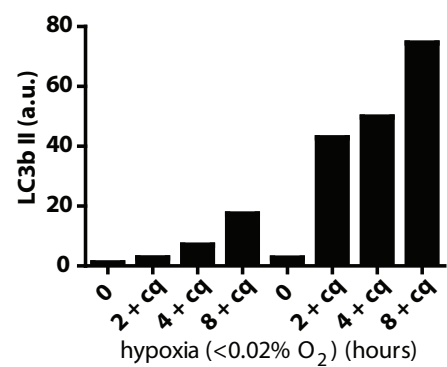

- ev + CQ

- $v i l l+C Q$

evhyp.

vlll hyp.

- evhyp.+CQ

口 vill hyp.+CQ

Figure 2. (A) EGFRvIll cells display increased autophagic flux under severe hypoxia (left panel, right panel is quantification of LC3b II). (B) EGFRvIll cells survive slightly better under severe anoxia compared to control cells but depend much more on their autophagy for survival.

response compared to the isogenic control cells as indicated by faster LC3b-II accumulation (Fig. 2A). To assess dependency on autophagy of EGFRvIII expressing cells during hypoxia, clonogenic survival after hypoxia exposure was determined. As expected, reduced survival during severe hypoxia was observed in both cell lines. However, EGFRvIll cells displayed a survival advantage over control cells (Fig 2B, grey lines). Addition of $C Q$ during these conditions had no effect on survival of the control cells. Strikingly, EGFRvill cells were greatly sensitized to severe hypoxia (Fig 2B, open symbols). These data indicate that EGFRvIll cells are more dependent on autophagy hypoxic conditions.

EGFRvIll xenografts depend on autophagy for accelerated growth?

As shown previously ${ }^{12}$, EGFRvIll tumors grow faster than control tumors (Fig. 
a

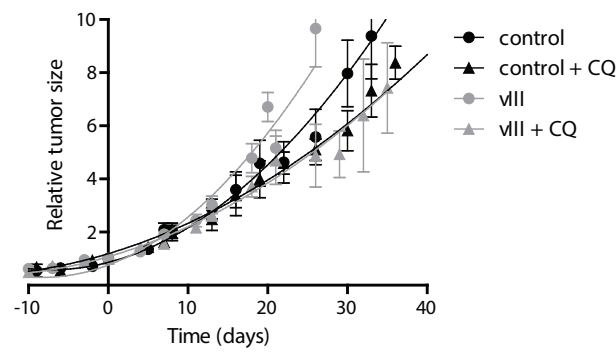

c

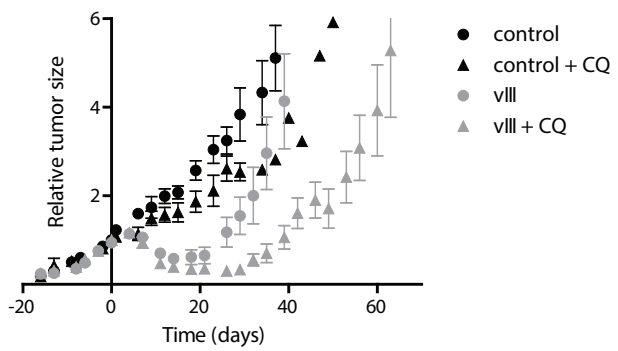

e

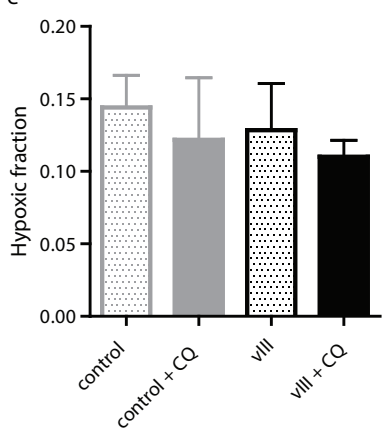

b

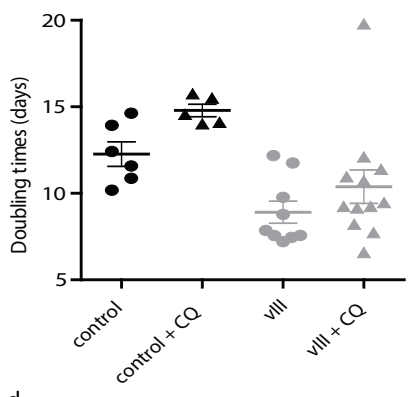

d

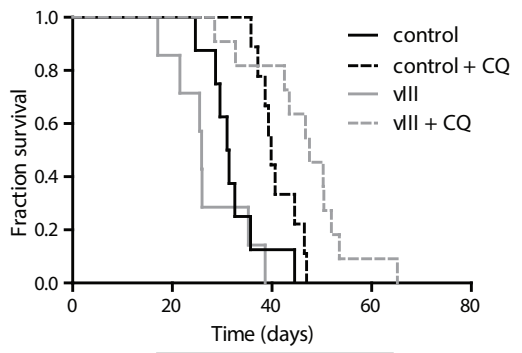

vill vs vill $+C Q, p<0.0001$ ev vs ev $+C Q, p=0.01$

f

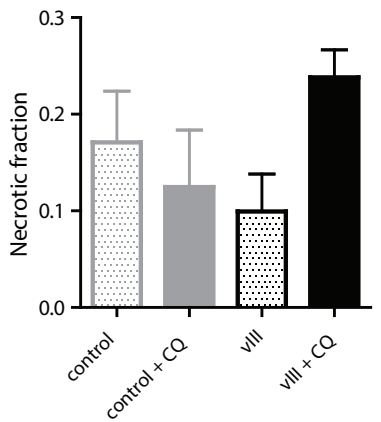

Figure 3. (A) EGFRvIII tumors grow faster than control but this can be abrogated by $C Q$ treatment by which EGFRvIII tumors' growth rate is reduced to control tumors' growth rate. (B) EGFRvIII tumors display a faster doubling time compared to control tumors, but tumor doubling times are reduced when treated with $C Q$. However, control tumors are more affected in their growth than EGFRvIll tumors suggesting that the targeted cells, most likely hypoxic cells, are not the main contributor to tumor growth. (C) EGFRvIll tumors regrow faster after radiotherapy but this can be overcome but $C Q$ treatment (D) Time to regrow to eight times the irradiated volume after irradiation with 10Gy. (E-F) Hypoxic and necrotic fraction of EGFRvIll and control tumors. (control $n=6$, control $+C Q n=6$, EGFRvIII n=5, EGFRvIII + CQ n=5) 
3A. To investigate if our in vitro findings were also valid in vivo, CQ was administered to tumor bearing animals. Interestingly, treatment with $C Q$ for seven days resulted in a small decrease in growth as also indicated by an increase in tumor doubling time in all tumors (Fig. 3B). Unexpectedly, the tumor volume doubling times of control tumors was relatively more increased than EGFRvIll tumors (20\% for control tumors vs $16.8 \%$ for EGFRvIll tumors)(Fig. $3 b)$. Nevertheless these changes are moderately suggesting that the targeted cells, most likely hypoxic cells, are not the main contributor to tumor growth. These findings are in line with earlier data. ${ }^{22,27}$

In contrast to the effects on growth, we previously showed that $C Q$ treatment sensitizes tumors to irradiation by decreasing the hypoxic fraction. ${ }^{22}$ Our in vitro data suggests that EGFRvIII expressing cells are highly dependent on autophagy for their survival during hypoxic exposure (Fig. 1B).

As hypoxic cells contribute to radiotherapy resistance, we determined if EGFRvIII expressing tumors could be sensitized to irradiation. Therefore we treated EGFRvIII and control tumor bearing mice with $\mathrm{CQ}$ for 7 days prior to irradiation and monitored tumor growth. We observed that control tumors hardly showed a growth delay after a single dose of 10Gy but could be radio sensitized by $C Q$ treatment (Fig. 3C, black symbols). EGFRvIII tumors were much more

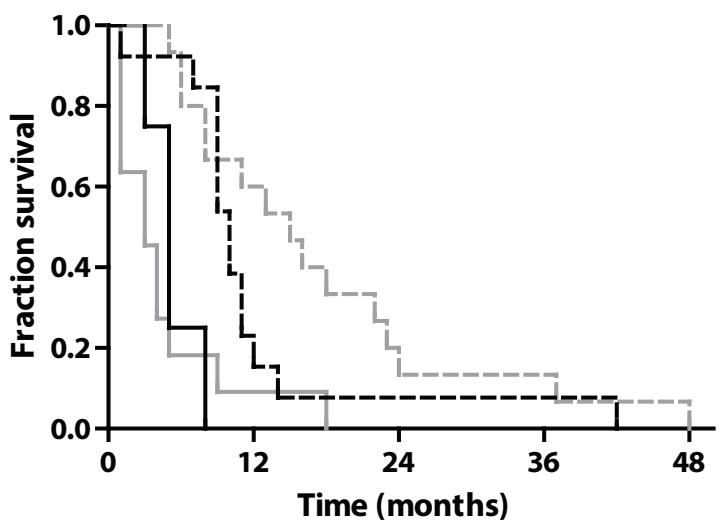

$\begin{array}{ll}\longleftarrow & \text { EGFRVIII + } \\ \text { ᄂ } & \text { EGFRVIII - } \\ \text {-ᄂ. } & \text { EGFRVIII + +CQ } \\ \text { ーட. } & \text { EGFRVIII - +CQ }\end{array}$

Figure 4. Survival plot of $43 \mathrm{GBM}$ patients categorized for EGFRvIll status and CQ treatment. Patients with an EGFRvIII positive tumor benefit substantially from CQ treatment. 
affected by radiotherapy than control tumors but regrew drastically faster. $\mathrm{CQ}$ treatment of EGFRvIII tumors postponed the regrowth even longer (Fig. 3C, grey symbols). When the time to reach $4 x$ the irradiated was plotted we observed no difference between EGFRvIll tumors and control tumors (Fig. 3D). $C Q$ treatment prior to radiotherapy postponed the regrowth of control tumors significantly. However, EGFRvIll tumors were even more delayed in their regrowth, suggesting that EGFRvIll tumors can be radio sensitized by $C Q$ treatment. This effect is even more pronounced after a single dose of 15Gy (Fig. S2). To determine whether the enhanced radio sensitizing effect in EGFRvIll-tumors was related to a more pronounced decrease in hypoxic fraction compared to the control tumors, tumor sections were stained for tumor hypoxia (pimonidazole). No differences were observed between groups (Fig. $3 \mathrm{E})$. The BrdU labeling index showed more BrdU incorporation in EGFRvIII tumors compared to control tumors (Fig. S3A), but CQ treatment did not affect the proliferation of these tumor cells. The vessel density in control tumors is increased probably due to vessel normalization as a consequence of $C Q$ treatment (Fig. S3B). Contradictory, EGFRvIll tumors showed a high vessel density when untreated. $C Q$ treatment reduced the vessel density to the level of control tumors treated with $C Q$. We could not detect a difference in perfused fraction of these tumors (Fig. S3C), this in contrast to the necrotic fraction (Fig. $3 F)$. The necrotic fraction of $C Q$ treated tumors was drastically increased compared to untreated tumors suggesting that $C Q$ treatment indeed contributes to the killing of tumors cells. Taken together, $C Q$ treatment is a promising treatment of rapidly growing EGFRvIII tumors.

Patients with an EGFRvIII positive GBM benefit from CQ treatment

To assess the clinical relevance of our findings we retrospectively analyzed 43 GBM patients, of which 28 patients received $C Q$ in combination with conventional treatment. EGFRvIll expression was assessed immunohistochemically and observed in 22 of 43 patients. In line with previous reports ${ }^{28,29}$, EGFRvIll expression was associated with poor survival (Fig. 4). CQ treatment improved median survival from 5 to 10 months in patients with EGFRvIII negative GBM. Strikingly median survival of patients with EGFRvIII 
positive GBM increased from 3 to 15 months, confirming the therapeutic potential $C Q$ in treatment of EGFRvIll expressing tumors.

\begin{tabular}{lll|ll}
\multicolumn{2}{c|}{ no CQ treatment } & \multicolumn{2}{l}{ CQ treatment } \\
\cline { 2 - 5 } EGFRvIII status & vIII - & vIII + & vIII - & VIII + \\
\cline { 2 - 5 } male & $5 / 8$ & $3 / 7$ & $10 / 13(77 \%)$ & $6 / 15(40 \%)$ \\
female & $3 / 8$ & $4 / 7$ & $3 / 13(23 \%)$ & $9 / 15(60 \%$ \\
Karnofsky preformace (pre-surgery) & & & & \\
$<70$ & $0(0 \%)$ & $1(14 \%)$ & $0(0 \%)$ & $1(7 \%)$ \\
$70-80$ & $7(87 \%)$ & $6(86 \%)$ & $7(54 \%)$ & $7(47 \%)$ \\
$90-100$ & $1(13 \%)$ & $0(0 \%)$ & $6(46 \%)$ & $7(47 \%)$ \\
Karnofsky preformace (post-surgery) & & & & \\
$<70$ & $1(13 \%)$ & $2(29 \%)$ & $0(0 \%)$ & $0(0 \%)$ \\
$70-80$ & $1(13 \%)$ & $1(14 \%)$ & $1(8 \%)$ & $3(20 \%)$ \\
$90-100$ & $6(74 \%)$ & $4(57 \%)$ & $12(92 \%)$ & $12(80 \%)$ \\
2 2nd surgery & $4 / 8(50 \%)$ & $2 / 7(29 \%)$ & $2 / 13(15 \%)$ & $2 / 15(13 \%)$ \\
\hline Radiotherapy & $3 / 8(38 \%)$ & $2 / 7(29 \%)$ & $8 / 13(62 \%)$ & $6 / 15(40 \%)$ \\
\hline
\end{tabular}

Table 1. Characteristics of GBM patients

\section{Discussion}

In this manuscript we showed that EGFRvIll expressing cells displayed increased autophagic flux during metabolic stresses like severe hypoxia and starvation. We also showed that EGFRvIll expressing cells proliferate faster and have increased survival under these stresses and that these advantages can be abrogated through inhibition of autophagy with CQ. In vivo, EGFRvIII tumors showed increased tumor growth and increased tumor regrowth after radiotherapy. However, $C Q$ treatment augmented the increased growth and regrowth of EGFRvIII tumors. Unexpectedly, CQ treatment did not change the hypoxic fraction of treated tumors, this in contrast with previous observations ${ }^{22}$, nor did $C Q$ treatment affect proliferation as expected from in vitro results. However, the necrotic fraction was drastically increased due to $C Q$ treatment of EGFRvIII tumors, suggesting that $C Q$ treatment is effective in EGFRvIII tumors.

The underlying mechanism for the increased autophagic flux in EGFRvIII expressing cells remains to be elucidated. One would expect reduced autophagic flux in EGFRvIII cells due to constitutive signaling of EGFRvIII through mTOR, a known autophagy inhibitor. However, preliminary results 
suggest that the RAS-RAF pathway ${ }^{30-32}$, one of the EGFR signaling pathways, plays a more important role in the increased flux in EGFRvIll cells (Fig. S4). Here we observed a block in autophagic flux when cell were treated with Vemurafenib (plx4032, a V600E BRAF inhibitor used at wtBRAF inhibiting concentration). ${ }^{33}$ Another possible explanation is the recently proposed hypothesis by Shen et.al. ${ }^{34}$; under normal conditions, latent cytoplasmic STAT3 binds to Protein Kinase $R(P K R)$ inhibiting its activity and reduces autophagy levels through elF2 $\alpha$ inhibition. In contrast, phosphorylated STAT3 forms dimers would leave PKR free to phosphorylate elF2 $\alpha{ }^{35}$ More recently, a new autophagy regulating mechanism has been described for EGFR; activated EGFR phosphorylates Beclin 1. Beclin 1 phosphorylation favors the formation of Beclin 1 dimers that are unable to bind VPS34 and promote autophagy. ${ }^{36}$ However, elucidating this mechanism warrants further investigation.

Most importantly, the effect of CQ on the outcome of patients with EGFRvIII expressing tumors was investigated. Analysis of the EGFRvIII staining revealed a drastically improvement in survival for patients with EGFRvIII positive GBM after concurrent CQ treatment (Fig. 4). Although these initial clinical results are promising, larger cohorts of patients are required to fully understand the therapeutic potential of CQ administration in treatment of GBM.

In conclusion, $\mathrm{CQ}$ treatment abrogates the growth and survival advantages of EGFRvIII expressing cells under metabolic stress and radio sensitizes EGFRvIII tumors for radiotherapy. Further, preliminary findings suggest that EGFRvIII positive cells regulate autophagy, at least in part, via the Ras-pathway, and patients with an EGFRvIII expressing tumor benefit from CQ treatment.

\section{References}

1. Lemmon MA, Bu Z, Ladbury JE, et al. Two EGF molecules contribute additively to stabilization of the EGFR dimer. EMBO J 1997;16:281-294.

2. Linggi B Carpenter G. ErbB receptors: new insights on mechanisms and biology. Trends in Cell Biology 2006;16:649-656.

3. Nicholson RI, Gee JM Harper ME. EGFR and cancer prognosis. Eur J Cancer 2001;37 Suppl 4:S9-15.

4. Dittmann K, Mayer C, Fehrenbacher B, Schaller M, Kehlbach R Rodemann HP. Nuclear EGFR shuttling induced by ionizing radiation is regulated by phosphorylation at residue 
Thr654. FEBS Lett 2010;584:3878-3884.

5. Schmidt-Ullrich RK, Mikkelsen RB, Dent P, et al. Radiation-induced proliferation of the human A431 squamous carcinoma cells is dependent on EGFR tyrosine phosphorylation. Oncogene 1997;15:1191-1197.

6. Ge H, Gong X Tang CK. Evidence of high incidence of EGFRvIII expression and coexpression with EGFR in human invasive breast cancer by laser capture microdissection and immunohistochemical analysis. Int J Cancer 2002;98:357-361.

7. Jutten B, Dubois L, Li Y, et al. Binding of cetuximab to the EGFRvIII deletion mutant and its biological consequences in malignant glioma cells. Radiother Oncol 2009;92:393398.

8. Okamoto I, Kenyon LC, Emlet DR, et al. Expression of constitutively activated EGFRvIII in nonsmall cell lung cancer. Cancer Science 2003;94:50-56.

9. Sok JC, Coppelli FM, Thomas SM, et al. Mutant epidermal growth factor receptor (EGFRvIII) contributes to head and neck cancer growth and resistance to EGFR targeting. Clin Cancer Res 2006;12:5064-5073.

10. Golding SE, Morgan RN, Adams BR, Hawkins AJ, Povirk LF Valerie K. Pro-survival AKT and ERK signaling from EGFR and mutant EGFRvIll enhances DNA double-strand break repair in human glioma cells. Cancer Biol Ther 2009;8:730-738.

11. Learn CA, Hartzell TL, Wikstrand CJ, et al. Resistance to tyrosine kinase inhibition by mutant epidermal growth factor receptor variant III contributes to the neoplastic phenotype of glioblastoma multiforme. Clin Cancer Res 2004;10:3216-3224.

12. Theys $\mathrm{J}$, Jutten $B$, Dubois $\mathrm{L}$, et al. The deletion mutant EGFRvIll significantly contributes to stress resistance typical for the tumour microenvironment. Radiother Oncol 2009;92:399-404.

13. Weppler SA, Li Y, Dubois L, et al. Expression of EGFR variant vlll promotes both radiationresistance and hypoxia tolerance. Radiother Oncol 2007;83:333-339.

14. Del Vecchio CA, Jensen KC, Nitta RT, Shain AH, Giacomini CP Wong AJ. Epidermal growth factor receptor variant III contributes to cancer stem cell phenotypes in invasive breast carcinoma. Cancer Res 2012;72:2657-2671.

15. Li G, Mitra SS, Monje M, et al. Expression of epidermal growth factor variant III (EGFRvIII) in pediatric diffuse intrinsic pontine gliomas. J Neurooncol 2012;108:395-402.

16. Liu XJ, Wu WT, Wu WH, et al. A minority subpopulation of CD133(+)/EGFRvIII(+) /EGFR(-) cells acquires stemness and contributes to gefitinib resistance. CNS Neurosci Ther 2013;19:494-502.

17. Gan HK, Kaye AH Luwor RB. The EGFRvIll variant in glioblastoma multiforme. J Clin Neurosci 2009;16:748-754.

18. Bigner SH, Humphrey PA, Wong AJ, et al. Characterization of the epidermal growth factor receptor in human glioma cell lines and xenografts. Cancer Res 1990;50:8017-8022.

19. Kondo Y, Kanzawa T, Sawaya R Kondo S. The role of autophagy in cancer development 
and response to therapy. Nat Rev Cancer 2005;5:726-734.

20. Ogier-Denis E Codogno P. Autophagy: a barrier or an adaptive response to cancer. Biochimica Et Biophysica Acta-Reviews on Cancer 2003;1603:113-128.

21. Mizushima N, Yoshimori T Levine B. Methods in Mammalian Autophagy Research. Cell 2010;140:313-326.

22. Rouschop KM, van den Beucken $\mathrm{T}$, Dubois $\mathrm{L}$, et al. The unfolded protein response protects human tumor cells during hypoxia through regulation of the autophagy genes MAP1LC3B and ATG5. J Clin Invest 2010;120:127-141.

23. Rouschop KM, Dubois L, Schaaf MB, et al. Deregulation of cap-dependent mRNA translation increases tumour radiosensitivity through reduction of the hypoxic fraction. Radiother Oncol 2011;99:385-391.

24. Guo D, Hildebrandt IJ, Prins RM, et al. The AMPK agonist AICAR inhibits the growth of EGFRvlll-expressing glioblastomas by inhibiting lipogenesis. Proc Natl Acad Sci U S A 2009;106:12932-12937.

25. Klionsky DJ, Abdalla FC, Abeliovich $\mathrm{H}$, et al. Guidelines for the use and interpretation of assays for monitoring autophagy. Autophagy 2012;8:445-544.

26. Klionsky DJ, Abeliovich $\mathrm{H}$, Agostinis $\mathrm{P}$, et al. Guidelines for the use and interpretation of assays for monitoring autophagy in higher eukaryotes. Autophagy 2008;4:151-175.

27. Rouschop KM, Dubois LJ, Keulers TG, et al. PERK/elF2alpha signaling protects therapy resistant hypoxic cells through induction of glutathione synthesis and protection against ROS. Proc Natl Acad Sci U S A 2013;110:4622-4627.

28. Pelloski $\mathrm{CE}$, Ballman KV, Furth $\mathrm{AF}$, et al. Epidermal growth factor receptor variant III status defines clinically distinct subtypes of glioblastoma. J Clin Oncol 2007;25:22882294.

29. Shinojima N, Tada K, Shiraishi S, et al. Prognostic value of epidermal growth factor receptor in patients with glioblastoma multiforme. Cancer Res 2003;63:6962-6970.

30. Guo JY, Chen HY, Mathew R, et al. Activated Ras requires autophagy to maintain oxidative metabolism and tumorigenesis. Genes Dev 2011;25:460-470.

31. Kim MJ, Woo SJ, Yoon CH, et al. Involvement of autophagy in oncogenic K-Ras-induced malignant cell transformation. J Biol Chem 2011;286:12924-12932.

32. Lock R, Roy S, Kenific CM, et al. Autophagy facilitates glycolysis during Ras-mediated oncogenic transformation. Mol Biol Cell 2011;22:165-178.

33. Bollag G, Tsai J, Zhang J, et al. Vemurafenib: the first drug approved for BRAF-mutant cancer. Nat Rev Drug Discov 2012;11:873-886.

34. Shen S, Niso-Santano M, Adjemian S, et al. Cytoplasmic STAT3 represses autophagy by inhibiting PKR activity. Mol Cell 2012;48:667-680.

35. Niso-Santano M, Shen S, Adjemian S, et al. Direct interaction between STAT3 and EIF2AK2 controls fatty acid-induced autophagy. Autophagy 2013;9:415-417.

36. Wei Y, Zou Z, Becker N, et al. EGFR-Mediated Beclin 1 Phosphorylation in Autophagy 
Suppression, Tumor Progression, and Tumor Chemoresistance. Cell 2013;154:12691284. 


\section{Supplementary figures}

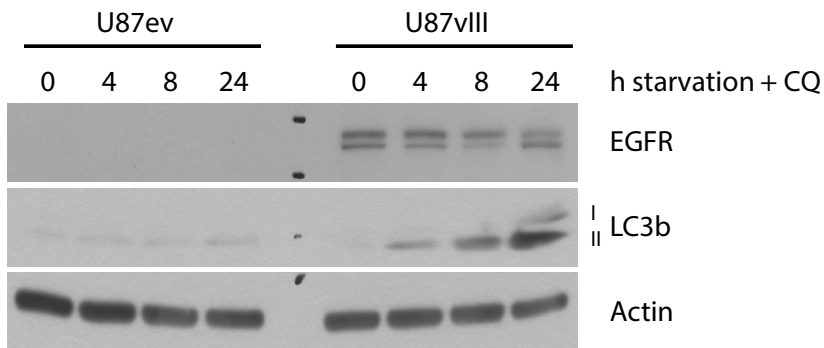

Figure S1. EGFRvIll expressing cells display increased autophagy under starvation; U-87 MG glioma cells

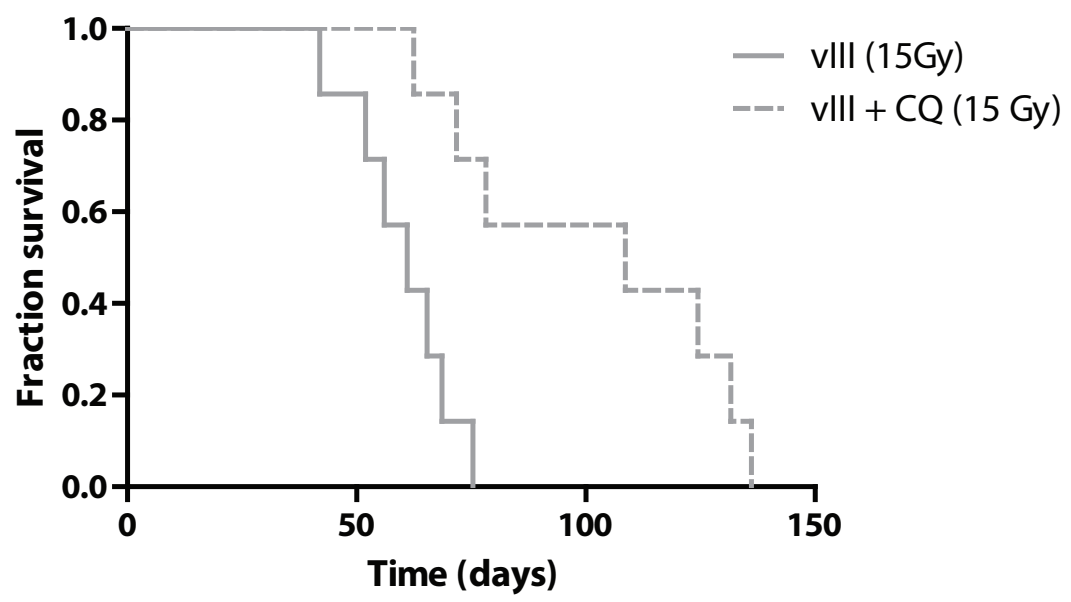

Figure S2. Time to regrow to eight times the irradiated volume after irradiation with $15 \mathrm{~Gy}$. 
a

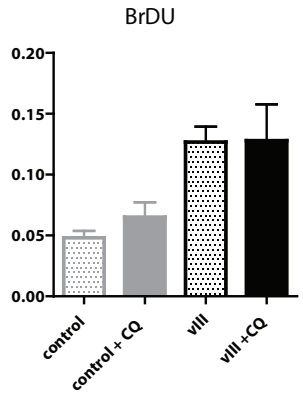

b

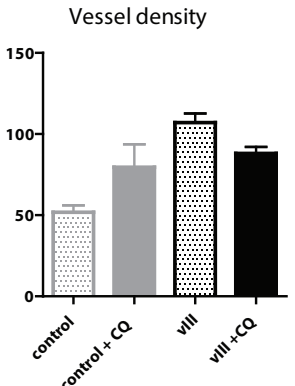

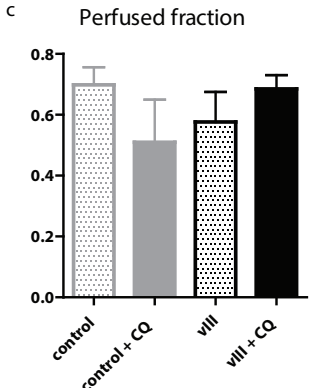

Figure S3. (A-C) Proliferation $(B r D U)(n=5,6,6,5)$, vessel density $(n=5,6,6,5)$ and perfused fraction $(n=5,4,3,2)$ of EGFRvIII and control tumors. $(n=$ control, control $+C Q$, vIII, vllI $+C Q)$

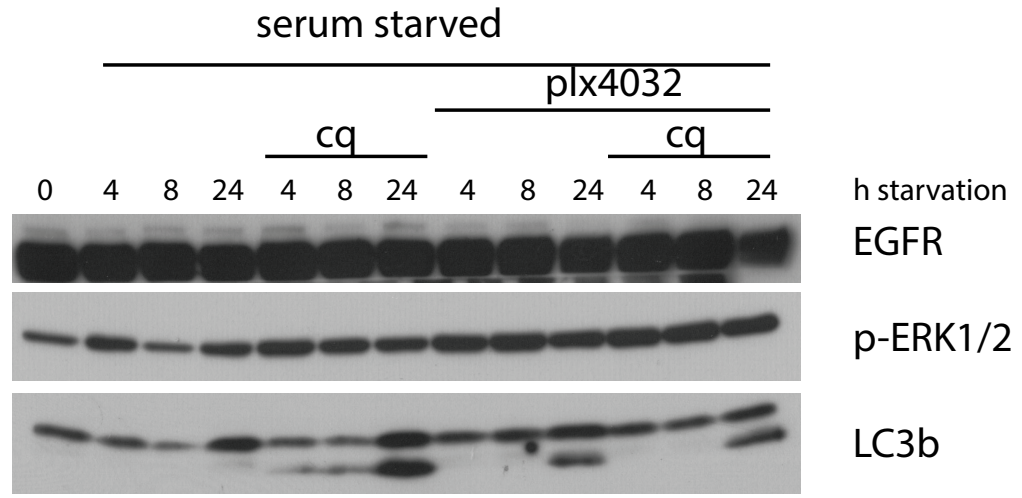

Figure S4. PLX4032 (Ras inhibitor) treatment blocks autophagic flux suggesting that Ras signaling is important for the autophagy process. 
$\longrightarrow$ 


\section{Chapter 6}

EGFR overexpressing cells and tumors are dependent on autophagy for growth and survival

Published in: Radiother Oncol. 2013 Sep;108(3):479-83

Jutten B, Keulers TG, Schaaf MB, Savelkouls K, Theys J, Span PN, Vooijs MA, Bussink J, and Rouschop KM 


\section{Abstract}

Background and purpose: The epidermal growth factor receptor (EGFR) is overexpressed, amplified or mutated in various human epithelial tumors, and is associated with tumor aggressiveness and therapy resistance. Autophagy activation provides a survival advantage for cells in the tumor microenvironment. In the current study, we assessed the potential of autophagy inhibition (using chloroquine (CQ)) in treatment of EGFR expressing tumors.

Material and methods: Quantitative PCR, immunohistochemistry, clonogenic survival, proliferation assays and in vivo tumor growth were used to assess this potential.

Results: We show that EGFR overexpressing xenografts are sensitive to $C Q$ treatment and are sensitized to irradiation by autophagy inhibition. In HNSSC xenografts, a correlation between EGFR and expression of the autophagy marker LC3b is observed, suggesting a role for autophagy in EGFR expressing tumors. This observation was substantiated in cell lines, showing high EGFR expressing cells to be more sensitive to $C Q$ addition as reflected by decreased proliferation and survival. Surprisingly high EGFR expressing cells display a lower autophagic flux.

Conclusions: The EGFR high expressing cells and tumors investigated in this study are highly dependent on autophagy for growth and survival. Inhibition of autophagy may therefore provide a novel treatment opportunity for EGFR overexpressing tumors. 


\section{Introduction}

The epidermal growth factor receptor (EGFR) is overexpressed, amplified or mutated in various human epithelial tumors. ${ }^{1}$ Its expression and activation leads to activation of several downstream effectors that control cell proliferation, differentiation, survival and acquisition of an EMT-like phenotype..$^{2,3}$ Consistently, EGFR amplification is associated with increased radioresistance through activation of pro-survival signaling and DNA-repair mechanisms. ${ }^{4-7}$ EGFR targeting is therefore regarded as a potential treatment strategy to sensitize tumors to therapy. ${ }^{8-10}$

Besides the intrinsic radiosensitivity of tumor cell, the tumor microenvironment has also influence on the tumor's responsiveness to irradiation. This microenvironment is characterized by extreme heterogeneity in oxygenation ranging from normal values close to vessels to complete anoxia in the perinecrotic regions. Poorly oxygenated (hypoxic) cells are highly resistant to chemoand radiotherapy. Therefore, targeting the hypoxic cells is likely to improve treatment efficacy. ${ }^{11-13}$ Tumor cells respond to hypoxia by activating a variety of different mechanisms, one of the most important ones being autophagy. Autophagy ("to eat oneself") is a lysosomal degradation mechanism that allows recycling of proteins and nutrients and is upregulated in response to various stresses, including hypoxia. ${ }^{13,14}$ During the initiation of autophagy phosphatidylethanol-amine (PE) is conjugated to MAP1LC3B (LC3b), allowing autophagic membrane association. Fusion with a lysosome allows degradation of the autophagosomal content but simultaneously degrades part of the LC3b associated with the inner membrane of the autophagosome..$^{15,16}$ Consistent with this, degradation of LC3b is considered the golden standard for autophagy. ${ }^{15}$ $\mathrm{CQ}$, a lysosomotropic compound, accumulates in the lysosomes and raises intralysosomal $\mathrm{pH}$, thereby preventing fusion autophagosome lysosome fusion. Previously we observed sensitization of tumors to irradiation after CQ administration through reduction of the hypoxic fraction. ${ }^{14}$ As EGFRexpressing tumors are radioresistant and require novel additions to increase tumor responsiveness, we explored the use of $C Q$ in treatment of EGFR overexpressing tumors. Interestingly, we observed not only a radiosensitizing effect as observed previously, but revealed dependency of EGFR expressing 
tumors on autophagy to maintain accelerated growth and survival. Autophagy inhibition may thus provide a novel treatment opportunity to target EGFR overexpressing tumors.

\section{Materials and methods}

Reagents and cell lines

Unless specified otherwise, all reagents were obtained from Sigma Chemical Co. (Sigma-Aldrich) and all electrophoresis reagents were from BioRad. MEMa medium was obtained from Invitrogen, glutamax-I (35050-038) was obtained from Life Technologies, DMEM and fetal bovine serum (FBS) was from PAA. The U373-MG, T47D, HT29 and A431 cell lines were obtained from ATCC and the E2 glioma cell line was kindly provided by A. Chalmers (Beatson Institute for Cancer Research \& Beatson West of Scotland Cancer Centre, University of Glasgow). Cells were maintained as described by ATCC, E2 cells were maintained in MEMa supplemented with $2 \mathrm{mM}$ glutamax-I.

Tumor xenograft model

Animal facilities and experiments were in accordance with local institutional guidelines and approved by the local animal welfare committee. Experiments were performed as described previously. ${ }^{17}$ Briefly, tumors were grown in NMRI$\mathrm{nu}(\mathrm{nu} / \mathrm{nu})$ female mice. U373-EGFRwt and U373 control cells in matrigel were injected subcutaneously $\left(1.5^{*} 10^{6}\right.$ cells). Tumor size was assessed by caliper measurement in 3 orthogonal diameters. Mice treated with $C Q$ received 60 $\mathrm{mg} / \mathrm{kg} \mathrm{CQ}$ for 7 consecutive days administered IP. Tumors were positioned in the irradiation field using a custom-built jig and irradiated with a single dose of $10 \mathrm{~Gy}(15 \mathrm{MeV}$ e) using a linear accelerator (Varian).

Immunohistochemistry and image processing

Frozen, acetone-fixed sections were stained by using antipimonidazole (Chemicon), anti LC3b (Abgent, AP1806a) and anti EGFR (Santa Cruz, sc-03). 
For quantitative analysis, the slides were scanned by a computerized digital image processing system by using a high-resolution intensified solid-state camera on a fluorescence microscope (Axioskop; Zeiss) with a computercontrolled motorized stepping stage. Tumor necrosis was evaluated, relative to the total tumor area, morphologically by using H\&E staining. Tumor hypoxic fraction and vascular density (structures per square millimeter) were determined relative to the viable tumor tissue (necrosis excluded).

Quantitative real-time PCR

RNA extraction was performed using the NucleoSpin RNA II kit (Bioke). cDNA was prepared using the iScript cDNA Synthesis kit (BioRad). Reactions were carried out in a 25 II volume using sensiMix SYBR low-ROX kit (GC Biotech) with the ABI Prism 7500 Sequence Detection System. Values for each gene were normalized to expression levels of RPL13a RNA. The primer sequences used were:

EGFR for: ACCTGCGTGAAGAAGTGTCC

EGFR rev: CGTTACACACTTTGCGGCAAGG

LC3b for: AACGGGCTGTGTGAGAAAAC

LC3b rev: AGTGAGGACTTTGGGTGTGG

RPL13a for: CCGGGTTGGCTGGAAGTACC

RPL13a rev: CTTCTCGGCCTGTTTCCGTAG

Clonogenic assay

After seeding and allowing cells to attach, indicated concentrations of $C Q$ were added to the medium and incubated for $96 \mathrm{~h}$. After standard incubation formed colonies were fixed $(0.4 \%$ methylene blue in $70 \%$ ethanol) and colonies consisting of $>50$ cells were counted manually.

Cellular proliferation

Cells were plated in a 24 well cell culture plate (Greiner) and allowed to attach to the plate. $C Q$ was added to the medium in the indicated concentration and 
cell densities were measured for 5 days using an IncuCyte ${ }^{\mathrm{TM}}$ Live-Cell Imaging System (SelectScience).

Western blotting

Cells were lysed and processed as described previously ${ }^{18}$ and proteins were separated on mini-PROTEAN precast gels (4-20\%, BIORAD). After transfer, proteins were probed with antibodies against EGFR (Santa-Cruz, sc-03), MAP1LC3b (Cell Signaling, 2775S), and Actin (MP Biomedicals, 8961001). Bound antibodies were visualized using HRP-linked anti-rabbit (Cell Signaling, 7074S) or anti-mouse (Cell Signaling, 7076S) antibodies.

\section{Results}

EGFR is often overexpressed in Glioblastoma Multiforme, which contributes to their radioresistant phenotype. ${ }^{4-6}$ Previously, we have shown that treatment of tumors with chloroquine $(\mathrm{CQ})$ decreases the hypoxic fraction and sensitizes them to radiation. ${ }^{14}$ To assess if similar results could be obtained in EGFR overexpressing tumors, U373-MG with constitutive EGFR overexpression were implanted in mice. As described before, these EGFR overexpressing tumors were highly resistant to irradiation as reflected by rapid regrowth after irradiation (single dose, $10 \mathrm{~Gy}$ ) (Fig. 1A and B). As observed previously, CQ administration sensitized tumors to irradiation and increased radiation-induced growth delay. Yet the effect of $C Q$ treatment alone was far more pronounced (Fig. 1A and $B$ ). In agreement with the tumor growth delay, the individual doubling time of EGFRexpressing tumors increased dramatically after $C Q$ administration, whereas the control tumors (U373-MG transfected with control vector) remained largely unaffected in proliferation (data not shown). These data indicate that EGFR overexpressing tumors are dependent on autophagy for growth. Although we previously observed a reduction of the hypoxic fraction in tumors after $C Q$ addition that explained the radiosensitizing effect of $C Q$ addition ${ }^{14}$, the large effect on growth of EGFR overexpressing tumors was neither due to decreased hypoxic fraction (Fig. 1D) ${ }^{14}$ nor due to differences in necrosis, apoptosis or proliferating cells (Fig. 1E-G). 

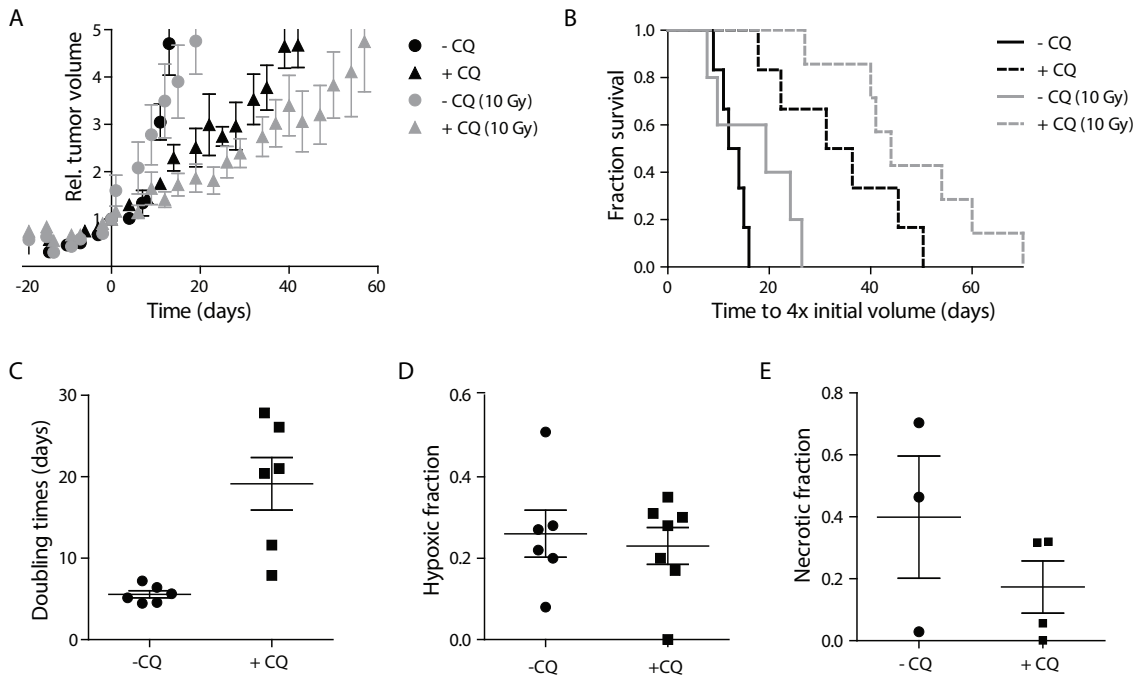

D
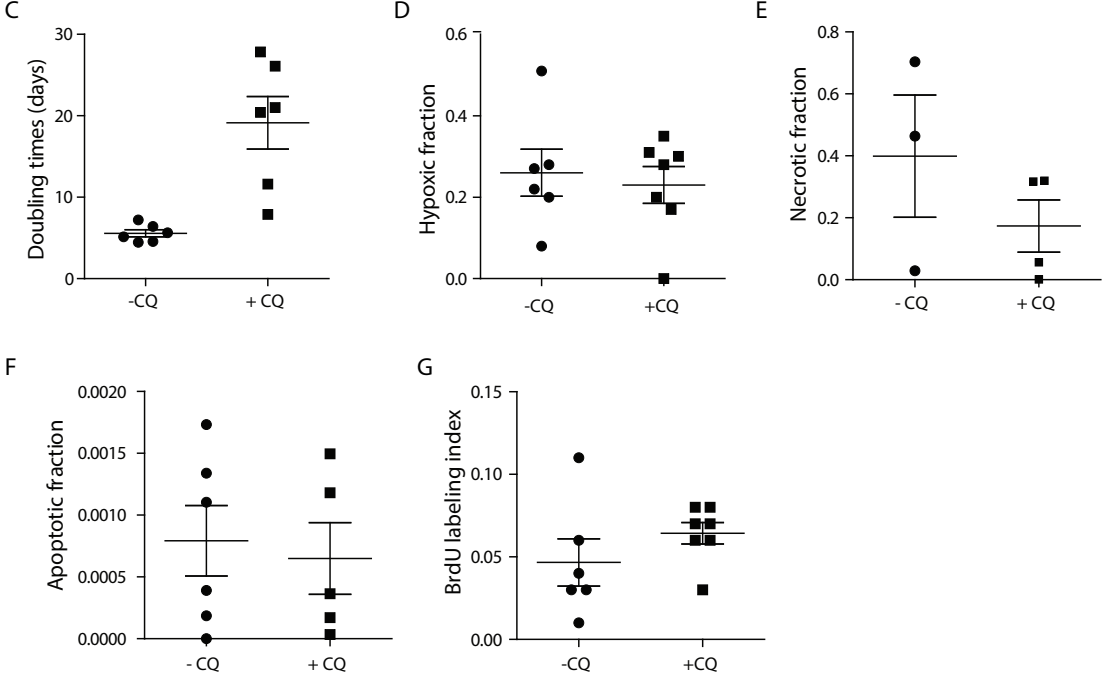

Figure 1. EGFR overexpressing U373-MG xenografts are sensitive to CQ treatment. (A) Growth of control $(C Q, n=6)$, irradiated $(C Q(10 G y), n=5), C Q$ treated $(+C Q, n=6)$ and irradiated in combination with $C Q$ treated (+CQ (10 Gy), $n=7)$ U373-MG xenografts with EGFR overexpression. (B) Time to reach 4 initial volume was plotted for the individual xenografts. Control (CQ) vs. $C Q$ treated $(+C Q), p<0.05$. Irradiated $(C Q(10 \mathrm{~Gy}))$ vs. irradiated in combination with $C Q(+C Q(10$ Gy)), $p<0.05$. (C) Tumor volume doubling times, (D) Hypoxic fraction, (E) Necrotic fraction (F) Apoptotic fraction, $(G)$ Brdu labeling index of the individual xenografts.

To determine whether a relation between EGFR expression and autophagy dependency also existed in other tumor types we performed immunohistochemical analysis of EGFR and LC3b in a panel of human head and neck squamous cell carcinoma (HNSCC) primary xenografts. Interestingly, we observed a correlation between EGFR and LC3b protein expression (Fig. $2 A)$. As LC3b has been reported to be primarily expressed in the hypoxic tumor regions, we assessed the correlation between EGFR and LC3b in the hypoxic (pimonidazole positive) area of the tumor (Fig. S1a) and between EGFR 
expression and the hypoxic fraction (Fig. S1b). However, no correlations were observed. We did find a correlation in mRNA abundance between EGFR and LC3b, while this was absent for EGFR and CA IX, a known hypoxia regulated gene (Fig. S1c). These findings suggest that EGFR is associated with expression and transcriptional regulation of LC3b in a non-hypoxia dependent manner. In agreement, immunohistochemical analysis of primary HNSCC xenografts revealed high LC3b expression in hypoxic regions in lowest EGFR expressing tumors, whereas in highest EGFR expressing tumors most LC3b was localized within the non-hypoxic regions (Fig. 2C).

To further address the high dependency of EGFR expressing cells on autophagy we explored the effect of $\mathrm{CQ}$ addition on cellular survival and proliferation. In agreement with our in vivo findings, U373-EGFR cells were more dependent on autophagy for survival and proliferation than U373 control

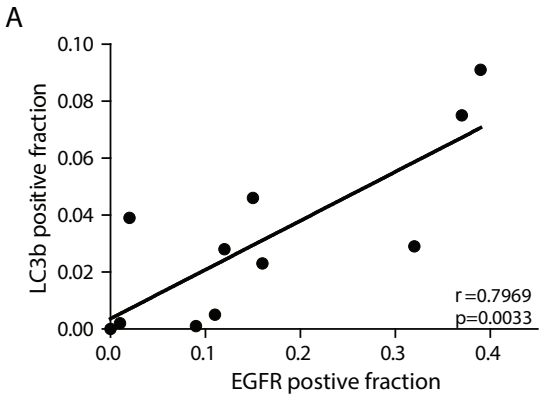

C

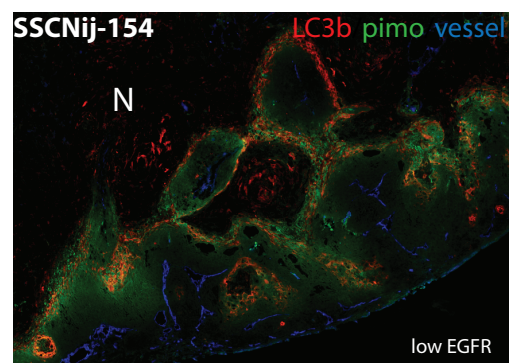

B
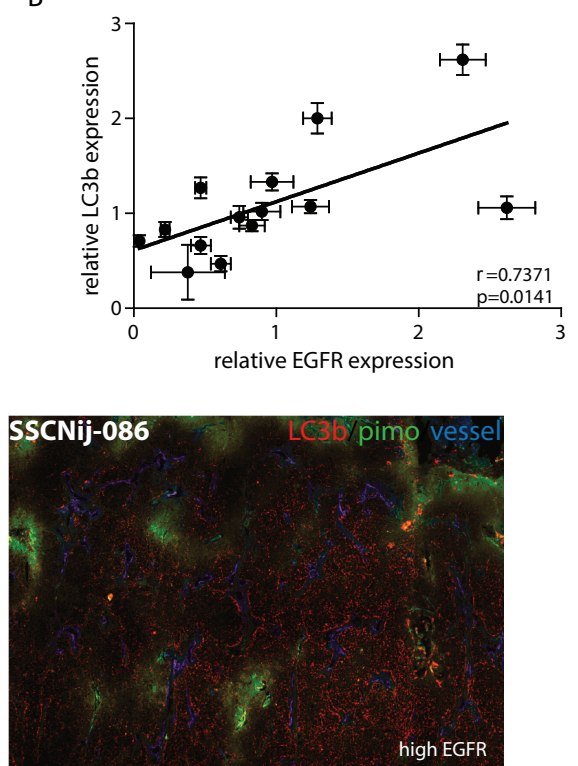

Figure 2. EGFR expression correlates with LC3b expression. (A) Correlation between LC3b positive fraction and EGFR positive fraction determined by IHC in a panel of primary HNSCC xenografts. (B) Correlation between EGFR and LC3b mRNA in a panel of primary HNSCC xenografts. (C) IHC of HNSSC with low EGFR (left panel) and high EGFR (right panel) expression, green: hypoxic fraction, blue: vessels and red: LC3b. N indicates necrotic area. 
cells as assessed by clonogenic survival (Fig. 3A) and cellular proliferation (Fig. 3B).

We observed that low $C Q$ concentrations reduced proliferation without reducing survival, suggesting that high EGFR expressing cells are highly dependent on cell cycle progression e.g. through maintaining high energy and nutrient availability. To investigate the high autophagy dependency further, we used a panel of high and low EGFR expressing cell lines (Fig. 3E) and tested their sensitivity to $C Q$ by clonogenic survival (Fig. 3C). We observed that high EGFR expressing cells (HT29 and A431) were more sensitive to $C Q$ compared to low EGFR expressing cell lines (E2 and T47D). This was confirmed by proliferation assessment (Fig. 3D) where CQ addition to EGFR high expressing cells led to a larger reduction in proliferation than in EGFR low expressing cells.

To determine if the high autophagy dependence of EGFR expressing cells was reflected by a high autophagic activation, autophagic flux was determined under normal growth conditions (flux is represented by differences in the amount of LC3-II between samples in the presence and absence of lysosomotrophic compounds ${ }^{16}$, like CQ). Unexpectedly we observed higher flux in the low EGFR expressing cells. These results indicate that although a lower autophagic flux in high EGFR expressing cells is observed, their dependency on autophagy for proliferation and survival is higher. The underlying reason for this observation remains to be elucidated.

\section{Discussion}

Our data presented here show that CQ drastically reducesis growth of EGFR overexpressing U373-MG xenografts and that this is not due to the previously described reduction of the hypoxic fraction. ${ }^{14} \mathrm{~A}$ possible explanation for this discrepancy could be the overexpression of EGFR which was not taken into account in our previous study. ${ }^{14}$ Neither the necrotic nor the apoptotic areas of xenografts are affected by $C Q$ treatment, suggesting that not the oxygen deprived cells are targeted but other cells that depend on autophagy, e.g. nutrient deprived cells. Additionally, in primary HNSCC xenografts we observed a positive correlation between LC3b and EGFR protein expression. In the same HNSCC xenografts we observed a correlation between LC3b and EGFR mRNA 

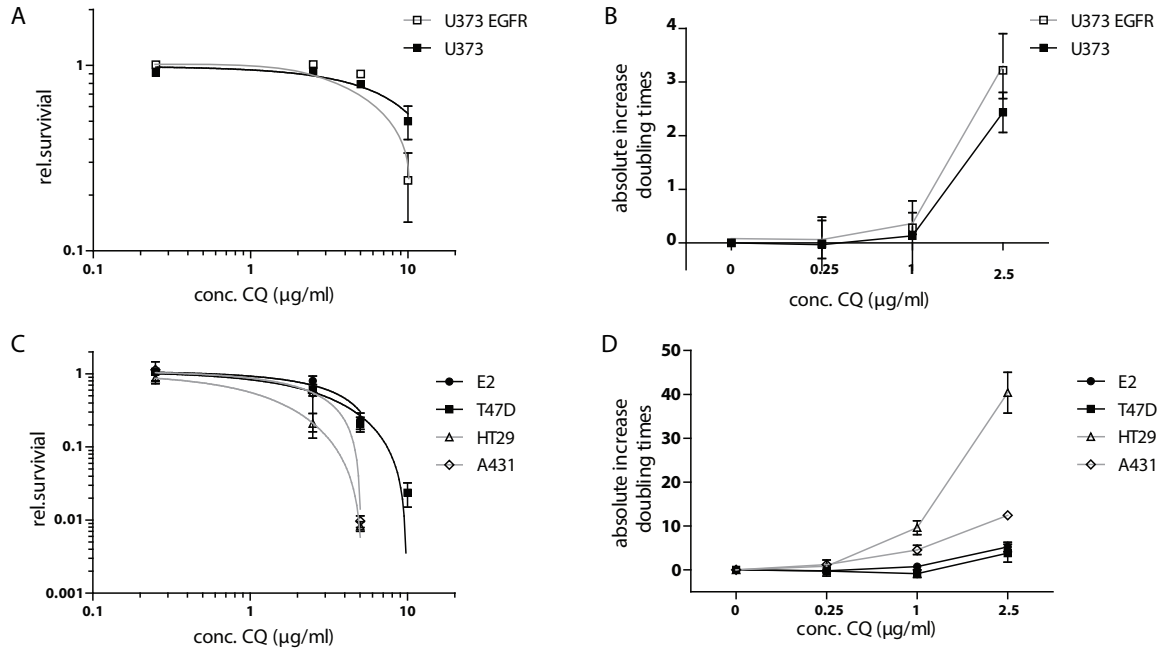

E
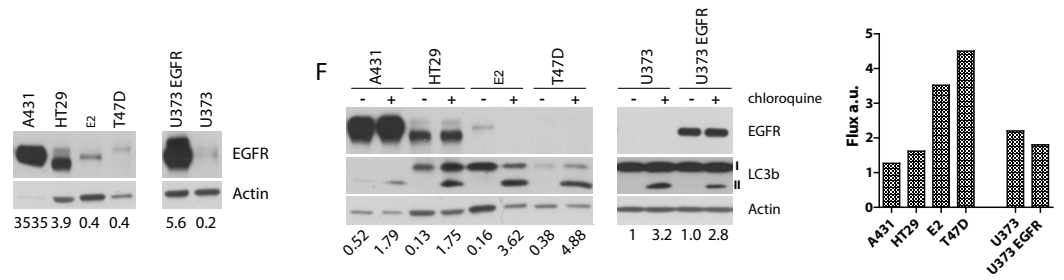

Figure 3. EGFR high expressing cells are more sensitive to $C Q$ treatment than EGFR low expressing cells (A) Dose-dependent clonogenic survival of EGFR overexpressing and control U373-MGs after CQ addition. (B) Doubling times of EGFR high and low U373-MGs after exposure to $C Q$. (C) Clonogenic survival of a panel of EGFR high and low cells after exposure to CQ. (D) Doubling times of panel of EGFR high and low cells after exposure to CQ. (E) WB showing total amount of EGFR normalized to actin expression. (F) Representative WB assessing autophagic flux under normal conditions. (Quantification of LC3b-II levels normalized to actin expression). (A$C$ and $D) n=4$ and SEM is plotted.

expression, suggesting a role for EGFR in the production of LC3b. Although in most tumors LC3b is predominantly expressed in hypoxic regions, LC3b expression in EGFR expressing tumors displayed no correlation with tumor hypoxia. In agreement, most LC3b in high EGFR expressing HNSCC xenografts was located within the non-hypoxic regions. In vitro experimentation in a panel of high and low EGFR expression cell lines revealed a relation between EGFR expression and CQ sensitivity under normal cell growth conditions. Based on our in vivo findings, the in vitro sensitization of U373 with 
EGFR overexpression is not as high as expected. This may be due to the artificial introduction of EGFR, survival and proliferation is thus not dependent on EGFR overexpression in vitro, but provides a growth advantage in vivo. All other cell lines with endogenous EGFR expression levels behaved in line with our findings; high EGFR expression, high sensitivity for CQ. Strikingly, EGFR high expressing cells showed remarkable low flux compared to EGFR low expressing cells under normal growth conditions, indicating that flux does not necessarily reflect the cells' dependency on autophagy for its survival. In line with our findings, knocking down EGFR rapidly leads to activation of autophagy as a result of reduced glucose uptake ${ }^{19-21}$, providing a potential explanation for the dramatic effect observed after $C Q$ administration. Nevertheless other effects of $C Q$, besides inhibition of autophagy, should be considered.

Another potential explanation for the high sensitivity to $C Q$ and autophagy inhibition of EGFR high expressing cells and tumors could be the EGFR signaling to RAS. It has been described that cells depend on autophagy during Ras-mediated oncogenic transformation. ${ }^{22,23}$ Further, it has been described that cells with oncogenic Ras signaling require autophagy to maintain their oxidative metabolism and that down-regulation of essential autophagy proteins impaired cell growth. As cancers with Ras mutations have a poor prognosis, this "autophagy addiction" suggests that targeting autophagy is a valuable new approach to treat these aggressive cancers. ${ }^{24}$

Next to Ras-signaling, mTOR signaling also influences autophagy. ${ }^{25,26}$ In the presences of sufficient nutrients mTOR drives proliferation but inhibits autophagy. This could explain the low autophagic flux in EGFR overexpressing cells. Additional inhibition of flux by $C Q$ could be lethal for these cells. However, the potential benefits from CQ treatment of EGFR high expressing tumors and the underlying mechanisms require further investigation.

In conclusion, EGFR high expressing cells and tumors investigated in this study are highly dependent on autophagy for growth and survival. Inhibition of autophagy may therefore provide a novel treatment opportunity for EGFR overexpressing tumors. 


\section{Acknowledgements}

The authors would like to thank Natasja Lieuwes and Hans Peters for their excellent technical support. This work was financially supported by the Dutch Cancer Society (KWF Grants UM 2010-4714 and 2012-5506 to K.R.).

\section{References}

1. Herbst RS. Review of epidermal growth factor receptor biology. Int J Radiat Oncol Biol Phys 2004;59:21-6.

2. Holz C, Niehr F, Boyko M, et al. Epithelial-mesenchymal-transition induced by EGFR activation interferes with cell migration and response to irradiation and cetuximab in head and neck cancer cells. Radiother Oncol 2011;101:158-64.

3. Theys J, Jutten B, Habets R, et al. E-Cadherin loss associated with EMT promotes radioresistance in human tumor cells. Radiother Oncol 2011;99:392-7.

4. Dittmann K, Mayer C, Fehrenbacher B, Schaller M, Kehlbach R, Rodemann HP. Nuclear EGFR shuttling induced by ionizing radiation is regulated by phosphorylation at residue Thr654. FEBS Lett 2010;584:3878-84.

5. Huang W-C, Chen Y-J, Hung M-C. Implication of nuclear EGFR in the development of resistance to anticancer therapies. BioMedicine 2011;1:2-10.

6. Schmidt-Ullrich RK, Valerie K, Fogleman PB, Walters J. Radiation-induced autophosphorylation of epidermal growth factor receptor in human malignant mammary and squamous epithelial cells. Radiat Res 1996;145:81-5.

7. Dittmann K, Mayer C, Fehrenbacher B, Schaller M, Kehlbach R, Rodemann HP. Nuclear epidermal growth factor receptor modulates cellular radio-sensitivity by regulation of chromatin access. Radiother Oncol 2011;99:317-22.

8. Astsaturov I, Cohen RB, Harari PM. Clinical application of EGFR inhibitors in head and neck squamous cell cancer. Cancer Treat Res 2008;139:135-52.

9. Krause M, Baumann M. Clinical biomarkers of kinase activity: examples from EGFR inhibition trials. Cancer Metastasis Rev 2008;27:387-402.

10. Raju U, Riesterer O, Wang ZQ, et al. Dasatinib, a multi-kinase inhibitor increased radiation sensitivity by interfering with nuclear localization of epidermal growth factor receptor and by blocking DNA repair pathways. Radiother Oncol 2012;105:241-9.

11. Stegeman $\mathrm{H}$, Kaanders JH, van der Kogel AJ, et al. Predictive value of hypoxia, proliferation and tyrosine kinase receptors for EGFR-inhibition and radiotherapy sensitivity in head and neck cancer models. Radiother Oncol 2013;106:383-9.

12. Overgaard J. Hypoxic modification of radiotherapy in squamous cell carcinoma of the head and neck - a systematic review and meta-analysis. Radiother Oncol 2011;100:22- 
32.

13. Starmans $\mathrm{MH}$, Chu KC, Haider S, et al. The prognostic value of temporal in vitro and in vivo derived hypoxia gene-expression signatures in breast cancer. Radiother Oncol 2012;102:436-43.

14. Rouschop KM, van den Beucken T, Dubois $L$, et al. The unfolded protein response protects human tumor cells during hypoxia through regulation of the autophagy genes MAP1LC3B and ATG5. J Clin Invest 2010;120:127-41.

15. Kabeya Y, Mizushima N, Ueno T, et al. LC3, a mammalian homologue of yeast Apg8p, is localized in autophagosome membranes after processing. EMBO J 2000;19:5720-8.

16. Mizushima N, Yoshimori T. How to interpret LC3 immunoblotting. Autophagy 2007;3:5425 .

17. Theys J, Jutten B, Dubois L, et al. The deletion mutant EGFRvIII significantly contributes to stress resistance typical for the tumour microenvironment. Radiother Oncol 2009;92:399-404.

18. Jutten B, Dubois L, Li Y, et al. Binding of cetuximab to the EGFRvIll deletion mutant and its biological consequences in malignant glioma cells. Radiother Oncol 2009;92:393-8.

19. Weihua Z, Tsan R, Huang WC, et al. Survival of cancer cells is maintained by EGFR independent of its kinase activity. Cancer Cell 2008;13:385-93.

20. Dittmann K, Mayer C, Rodemann HP, Huber SM. EGFR cooperates with glucose transporter SGLT1 to enable chromatin remodeling in response to ionizing radiation. Radiother Oncol 2013;107:247-51.

21. Huber SM, Misovic M, Mayer C, Rodemann HP, Dittmann K. EGFR-mediated stimulation of sodium/glucose cotransport promotes survival of irradiated human A549 lung adenocarcinoma cells. Radiother Oncol 2012;103:373-9.

22. Lock R, Roy S, Kenific CM, et al. Autophagy facilitates glycolysis during Rasmediated oncogenic transformation. Mol Biol Cell 2011;22:165-78.

23. Kim MJ, Woo SJ, Yoon $\mathrm{CH}$, et al. Involvement of autophagy in oncogenic K-Rasinduced malignant cell transformation. J Biol Chem 2011;286:12924-32.

24. Guo JY, Chen HY, Mathew R, et al. Activated Ras requires autophagy to maintain oxidative metabolism and tumorigenesis. Genes Dev 2011;25:460-70.

25. Kim J, Kundu M, Viollet B, Guan KL. AMPK and mTOR regulate autophagy through direct phosphorylation of Ulk1. Nat Cell Biol 2011;13:132-41.

26. Nazio F, Strappazzon F, Antonioli M, et al. MTOR inhibits autophagy by controlling ULK1 ubiquitylation, self-association and function through AMBRA1 and TRAF6. Nat Cell Biol 2013;15:406-16. 
Chapter 7

General discussion and summary 


\section{General Discussion and Summary}

This thesis describes how the Epidermal Growth Factor Receptor (EGFR) and one of its most commonly observed mutated form, variant III (EGFRvIII), contribute to a more malignant tumor phenotype and describes possible new treatment opportunities for patients with deregulated EGFR signaling.

The EGFR is a receptor tyrosine kinase of the ErbB family, consisting of four members; EGFR (ErbB1, HER1), ErbB2 (HER2), ErbB3 (HER3) and ErbB4 (HER4). EGFR is activated by ligand binding to the extracellular ligand binding domain. This initiates a conformational change of the receptor and exposure of the dimerization domain, allowing homo-dimerization or hetero-dimerization with other family members and autophosphorylation by the intracellular kinase domain. EGFR activates a number of signaling cascade that drive many cellular responses, including changes in gene expression, cytoskeletal rearrangement, apoptosis inhibition, increased cell proliferation and acquisition of an EMT-like phenotype. ${ }^{1-3}$ Not long after their discovery in the early 1980 s the ErbB family members were implicated in cancer research. In many different cancer cell types, the ErbB pathway becomes hyper activated by a range of mechanisms, including overproduction of ligands, overproduction of receptors, or constitutive activation of receptors. ${ }^{4}$

Consistently, amplification of the EGFR gene is associated with increased radio resistance through activation of pro-survival signaling and DNA-repair mechanisms. ${ }^{5-7}$ Targeting EGFR is therefore regarded a potential treatment strategy to sensitize tumors to therapy. ${ }^{8-10}$ Despite many efforts that have been made to develop EGFR targeting therapies like inhibition of EGFRsignaling through tyrosine kinase inhibitors (TKI) and preventing EGFR expression and dimerization using antibodies, these therapies have only been proven effective in a limited percentage of patients. ${ }^{11}$

Recent data have emerged demonstrating that certain cancer-associated mutations, including k-Ras and PTEN can result in persistent growth pathways activation despite the blockade of EGFR ${ }^{12,13}$ and abrogate the efficacy antiEGFR-targeted treatments. Furthermore, it has also become apparent that different treatment strategies to inhibit EGFR (e.g. antibody versus TKI) yield different effects in the same tumor. ${ }^{9,14}$ Compensatory responses by other 
receptors, including mutant EGFR species, are likely reasons for such failures. The most common variant of the EGFR is an exon 2-7 deletion mutant EGFRvIII. ${ }^{15-17}$ EGFRvIll is known to contribute to the radio resistance of tumor cells ${ }^{18}$ at least in part through enhanced repair of DNA double strand breaks. ${ }^{19}$ Furthermore, EGFRvIII expression contributes to resistance to the TKI gefitinib and leads to sustained EGFR signaling and AKT activity. ${ }^{20}$

Additionally, the tumor microenvironment, and in particular the hypoxic tumor regions, significantly contribute to therapy resistance. ${ }^{21-23}$ Expression of EGFRvIll provides cells with a survival advantage when exposed to stresses as hypoxia and nutrient starvation. ${ }^{24}$ We therefore investigated whether EGFRvIll expressing tumor cells can be target with Cetuximab, a monoclonal antibody directed against EGFR. Cetuximab prevents binding of ligands to EGFR and as EGFRvIII lacks the majority of domain I and II (domain I and III are responsible for ligand binding) we questioned whether Cetuximab binds to EGFRvIII and whether Cetuximab binding to EGFRvIII would have functional therapeutic effects. Indeed, in chapter 2 we showed that Cetuximab binds to EGFRvIII resulting in a reduction of EGFRvIll expression on the cell membrane. Unexpectedly, this reduction had no effect on the proliferation rate of these EGFRvIII expressing glioma cells. Potentially, EGFRvIII expression is not beneficial for proliferation under normal cell growth conditions or the down regulation was insufficient to evoke phenotypic changes. In line, Cetuximab treatment also failed to radio sensitize these EGFRvIII expressing cells, again suggesting that, the residual EGFRvIll receptors left on the membrane are sufficient to maintain the radio resistant phenotype of these cells. Nevertheless, ERK signaling is reduced in these EGFRvIll expressing cells suggesting that Cetuximab reduces EGFRvIII signaling and could mediate therapeutic effects against EGFRvIII in cells with less extensive EGFRvIII expression.

Currently, EGFR expression and mutations in its tyrosine kinase domain are assessed for diagnosis and clinical treatment of patients. Also downstream effectors of EGFR like k-RAS and PTEN are routinely evaluated as k-RAS mutations can compromise Cetuximab efficacy and loss of PTEN ablates effectiveness of TKIs directed against EGFR. The expression of these proteins is therefore tested for optimal treatment. However, EGFRvIII is not routinely determined despite its clinical relevance. ${ }^{25,26}$ Additionally, EGFRvIll expression 
adversely effects anti-EGFR therapy. ${ }^{20}$ Cells expressing EGFRvIll are relatively resistant to gefitinib and require higher doses, repeated dosing, and longer exposure to achieve decreased receptor phosphorylation. However, this decrease does not effectively inhibit the biologically relevant processes of DNA synthesis, cellular growth, and invasion in cells expressing EGFRvIII. ${ }^{20} \mathrm{~A}$ potential explanation for not routinely testing EGFRvIll expression is the lack of commercially availability of an EGFRvIll specific antibody. We therefore attempted to develop an EGFRvIll specific llama derived antibody which could be used for in vitro and in vivo assessment (chapter 3) as llama single domain heavy chain only antibody fragments, or nanobodies, are in general suitable for both PET-imaging ${ }^{27}$ and detection on cryosections. ${ }^{28}$ This EGFRvIII nanobody could be used in combination with a previously selected EGFR specific nanobody (Roovers et al. unpublished results) to determine EGFR and EGFRvIII expression in vitro or in vivo and thereby helping to make the best suited treatment. Unfortunately, the selected nanobody against EGFRvIII showed also affinity for EGFR making this nanobody unsuitable to discriminate between EGFR and EGFRvIII expression as EGFRvIII expression is almost always accompanied with EGFR overexpression.

In chapter $\mathbf{4}$ we investigated the effect of EGFR and EGFRvIII expression on the stress resistance typical for the tumor microenvironment. We observed that EGFRvIII but not EGFR expressing cells maintained a higher proliferation potential during stress conditions as serum starvation or hypoxia. Similar, in the same model we also observed that EGFRvIll but not EGFR overexpressing cells displayed improved survival after exposure to nutrient starvation and hypoxia. Interestingly, the observed in vitro effects were also translated in increased tumor growth rates in vivo. We noted that the increased growth rate of EGFRvIll-expressing tumors could be reversed when the mutant receptor was no longer present. These data indicate that targeting EGFRvIII by silencing its expression and/or blocking its downstream effects might be a promising strategy for treating cancers that express this mutated receptor.

In chapter $\mathbf{5}$ we demonstrated that EGFRvIII expressing cells and tumors depend on autophagy to maintain their proliferative state and for their survival during metabolic stresses like starvation or severe hypoxia. We also showed that these advantages can be abrogated by inhibiting autophagy. As observed 
previously, EGFRvIll tumors displayed increased tumor growth and accelerated tumor regrowth after radiotherapy. Interestingly, chloroquine (CQ) treatment abrogated the growth and regrowth advantage of EGFRvIll tumors. The underlying mechanism for the increased autophagic flux in EGFRvIll expressing cells remains to be elucidated. Intuitively, one would expect reduced autophagic flux in EGFRvIll cells due to constitutive signaling of EGFRvIll through mTOR $^{29,30}$, a known negative regulator of autophagy. However, preliminary results suggest that the RAS-RAF pathway ${ }^{31-33}$, one of the EGFR signaling pathways, plays a more important role in the increased flux in EGFRvIll cells. Although autophagy is neither regulated via transcription nor translation, yet sufficient transcription and translation of autophagy related proteins is needed to sustain autophagic flux. ${ }^{34}$ Signaling through the Signal Transducer and Activator of Transcription 3 (STAT3) pathway could be responsible for sustaining the increased autophagic flux under hypoxic conditions of EGFRvIII expressing cells through Protein Kinase R (PKR) and elF2a. ${ }^{35,36}$ More recently, a fourth autophagy regulating mechanism has been described for EGFR. ${ }^{37}$ EGF activated EGFR phosphorylates Beclin 1 at three tyrosine residues favoring Beclin 1 to form dimers and thereby preventing binding of Beclin 1 to the Vps34 complex and initiation of autophagy. ${ }^{38,39}$ This may also explain why EGFR overexpressing cells display low basal autophagic flux as described in chapter 6. Nevertheless, to elucidate the exact mechanism at play more research is needed.

Furthermore, the effect of $C Q$ on the outcome of patients with EGFRvIII expressing Glioblastoma Multiforme (GBM) was investigated. Importantly, in cohort of 52 GBM patients (18 conventional treatment and 34 treated with additional $C Q$ ) large improvements in overall survival were observed. Patients harboring EGFRvIII negative tumors displayed an increase in median survival from 5 to 10 months, where patients with EGFRvIll positive tumors displayed an increase in median survival from 3 to 15 months. However, these results should be treated with caution since this group of patients is relative small and was treated heterogeneously. Nevertheless, these promising results should be further pursued. Taken together, inhibition of autophagy may provide a novel treatment opportunity for EGFRvIll expressing tumors.

To assess whether there is also a relation between non-mutated EGFR and 
autophagy we engrafted EGFR overexpression glioblastoma cells in a xenograft model and assessed CQ treatment efficacy, described in chapter 6 . We observed a reduction in growth of EGFR expressing xenografts when treated with $C Q$. As expected EGFR expressing tumors were highly radio resistant. Interestingly, within these radio resistant tumors were we also observed a radio sensitization effect after $C Q$ treatment, as also observed in EGFRvIll expressing xenografts (chapter 5). Interestingly, this was not due to the previously described reduction of the hypoxic fraction. ${ }^{34}$ Neither the necrotic nor the apoptotic areas of xenografts appear affected by $C Q$ treatment, suggesting that not only oxygen deprived cells are targeted but other cells, illustrating the high dependence of EGFR expressing cells on autophagy for survival. Additionally, in primary Head and Neck Squamous Cell Carcinoma (HNSCC) xenografts we observed a positive correlation between LC3b and EGFR protein expression. In the same HNSCC xenografts we observed a correlation between LC3b and EGFR mRNA expression, suggesting a role for EGFR in the production of LC3b. Although in most tumors LC3b is predominantly expressed in hypoxic regions, LC3b expression in EGFR expressing tumors displayed no correlation with tumor hypoxia, suggesting once more that other cells that depend on autophagy, e.g. nutrient deprived cells are targeted but not the oxygen deprived cells. In agreement, most LC3b in high EGFR expressing HNSCC xenografts was located within the nonhypoxic regions. Taken together, EGFR high expressing tumors investigated in this study are highly dependent on autophagy for growth, survival and radio resistance. Inhibition of autophagy may therefore provide a novel treatment opportunity for EGFR overexpressing tumors.

\section{Concluding remarks and future perspectives}

EGFR has become a highly investigated molecule since its first implication in cancer in the early 1980s. In many different cancer types, the EGFR pathway becomes hyper activated by a range of mechanisms, including overproduction of ligands, overproduction of receptors, or constitutive activation of receptors. ${ }^{4}$ As a consequence, EGFR and related signaling molecules are routinely tested for diagnostically purposes and treatment selection. Surprisingly, EGFRvIII 
expression is not routinely tested despite the increasing amount of research and clinical data that suggest that EGFRvIll expression adversely effects the therapy success and thereby the outcome for the patient and should be additionally tested to optimize patient treatment.

Many anti-EGFR therapies, like Tyrosine Kinase Inhibitors (TKI) or monoclonal antibodies (mAbs) have been proven effective in experimental settings; however show only little clinical efficacy. ${ }^{11}$ Tumors could potentially become resistant to anti-EGFR treatment by induction of compensatory mechanisms or expression of EGFRvIll. To overcome this resistance novel strategies are desired.

In this thesis we described a possible new treatment possibility for the treatment of EGFR and EGFRvIll overexpressing tumors. We have shown that both EGFR and EGFRvIll expressing tumors respond very well to autophagy inhibition through $C Q$ administration. Additionally, within a cohort of patients with $C Q$ in combination with conventional therapy, patients with EGFRvIII expressing GBM benefited most. Nevertheless, larger and more clinical trials with uniformly treated patients are required to evaluate effectiveness of $C Q$ addition in treatment of (EGFRvIll positive) GBM.

Unraveling the exact signaling mechanism how EGFR and EGFRvIII regulate autophagy, could create new insight on how these tumors could be treated more efficiently. Many inhibitors of downstream effectors of EGFR e.g. PI3K, mTOR, RAS or RAF are currently available for researchers or even already tested in clinical trial. These inhibitors in combination with anti-EGFR treatment could provide new treatment possibilities. Moreover, it has become clear that single therapies are not effective in most cancer treatments and combinations of existing therapies should be tested. In that perspective, GBM patients could be treated with Avastin (Bevacizumab, a mAb directed against Vascular Endothelial growth factor (VEGF) that inhibits angiogenesis) in combination with autophagy inhibition and radiotherapy (RT). Bevacizumab treatment effects are mainly mediated trough induction of tumor hypoxia. Combining Bevacizumab with $C Q$ is likely to increase cell killing and the effectiveness of Bevacizumab as single agent treatment but also to prevent or delay recurrence after irradiation. ${ }^{40}$

Another approach in targeting EGFRvIll expressing tumors is the use of cancer 
vaccination. ${ }^{41}$ EGFRvIII is the ideal cancer vaccination candidate as it is solely expressed on tumors and not on normal tissue. Moreover, vaccination of patients with an EGFRvIII positive GBM with an EGFRvIII peptide led to a 5fold improved overall survival. ${ }^{42}$ Unfortunately, Natural Killer (NK) cells are less effective in a hypoxic tumor environment ${ }^{43}$ and autophagy protects tumor cells from cytotoxic T-lymphocyte (CTL) mediated lysis. ${ }^{44}$ Combination of RT, CQ treatment and vaccination could therefore eradicate all normoxic and hypoxic tumors cells. This approach not only targets all 'normal' tumor cells but also the very radio resistant glioblastoma cancer stem cell (CSC) which express EGFRvIII. 45,46

Taken together, despite many decennia of research, new anti-EGFR drugs and clinical trials, EGFR and EGFRvIII expressing tumors remain difficult to treat and more research is required to elucidate the molecular mechanisms at play and to identify the treatment with highest efficacy.

\section{References}

1. Lemmon MA, Bu Z, Ladbury JE, et al. Two EGF molecules contribute additively to stabilization of the EGFR dimer. EMBO J 1997;16:281-294.

2. Linggi B Carpenter G. ErbB receptors: new insights on mechanisms and biology. Trends in Cell Biology 2006;16:649-656.

3. Theys J, Jutten B, Habets R, et al. E-Cadherin loss associated with EMT promotes radioresistance in human tumor cells. Radiother Oncol 2011;99:392-397.

4. Yarden Y Sliwkowski MX. Untangling the ErbB signalling network. Nat Rev Mol Cell Biol 2001;2:127-137.

5. Dittmann K, Mayer C, Fehrenbacher B, Schaller M, Kehlbach R Rodemann HP. Nuclear EGFR shuttling induced by ionizing radiation is regulated by phosphorylation at residue Thr654. FEBS Lett 2010;584:3878-3884.

6. Huang W-C, Chen Y-J Hung M-C. Implication of nuclear EGFR in the development of resistance to anticancer therapies. BioMedicine 2011;1:2-10.

7. Schmidt-Ullrich RK, Valerie K, Fogleman PB Walters J. Radiation-induced autophosphorylation of epidermal growth factor receptor in human malignant mammary and squamous epithelial cells. Radiat Res 1996;145:81-85.

8. Astsaturov I, Cohen RB Harari PM. Clinical application of EGFR inhibitors in head and neck squamous cell cancer. Cancer Treat Res 2008;139:135-152.

9. Baumann M, Krause M, Dikomey E, et al. EGFR-targeted anti-cancer drugs in radiotherapy: preclinical evaluation of mechanisms. Radiother Oncol 2007;83:238-248. 
10. Krause M Baumann M. Clinical biomarkers of kinase activity: examples from EGFR in hibition trials. Cancer Metastasis Rev 2008;27:387-402.

11. Harandi A, Zaidi AS, Stocker AM Laber DA. Clinical Efficacy and Toxicity of Anti-EGFR Therapy in Common Cancers. J Oncol 2009;2009:567486.

12. Jhawer M, Goel S, Wilson AJ, et al. PIK3CA mutation/PTEN expression status predicts response of colon cancer cells to the epidermal growth factor receptor inhibitor cetuximab. Cancer Res 2008;68:1953-1961.

13. Lievre A, Bachet JB, Boige $\mathrm{V}$, et al. KRAS mutations as an independent prognostic factor in patients with advanced colorectal cancer treated with cetuximab. Journal of Clinical Oncology 2008;26:374-379.

14. Krause M, Prager J, Zhou XJ, et al. EGFR-TK inhibition before radiotherapy reduces tumour volume but does not improve local control: Differential response of cancer stem cells and nontumourigenic cells? Radiotherapy and Oncology 2007;83:316-325.

15. Ge H, Gong X Tang CK. Evidence of high incidence of EGFRvIll expression and coexpression with EGFR in human invasive breast cancer by laser capture microdissection and immunohistochemical analysis. Int J Cancer 2002;98:357-361.

16. Okamoto I, Kenyon LC, Emlet DR, et al. Expression of constitutively activated EGFRvIII in nonsmall cell lung cancer. Cancer Science 2003;94:50-56.

17. Sok JC, Coppelli FM, Thomas SM, et al. Mutant epidermal growth factor receptor (EGFRvIII) contributes to head and neck cancer growth and resistance to EGFR targeting. Clin Cancer Res 2006;12:5064-5073.

18. Weppler SA, Li Y, Dubois L, et al. Expression of EGFR variant vIll promotes both radiation resistance and hypoxia tolerance. Radiother Oncol 2007;83:333-339.

19. Golding SE, Morgan RN, Adams BR, Hawkins AJ, Povirk LF Valerie K. Pro-survival AKT and ERK signaling from EGFR and mutant EGFRvIll enhances DNA double-strand break repair in human glioma cells. Cancer Biol Ther 2009;8:730-738.

20. Learn CA, Hartzell TL, Wikstrand CJ, et al. Resistance to tyrosine kinase inhibition by mutant epidermal growth factor receptor variant III contributes to the neoplastic phenotype of glioblastoma multiforme. Clin Cancer Res 2004;10:3216-3224.

21. Hockel M, Schlenger K, Aral B, Mitze M, Schaffer U Vaupel P. Association between tumor hypoxia and malignant progression in advanced cancer of the uterine cervix. Cancer Res 1996;56:4509-4515.

22. Nordsmark M, Bentzen SM, Rudat V, et al. Prognostic value of tumor oxygenation in 397 head and neck tumors after primary radiation therapy. An international multi-center study. Radiother Oncol 2005;77:18-24.

23. Wouters BG, van den Beucken T, Magagnin MG, Lambin P Koumenis C. Targeting hypoxia tolerance in cancer. Drug Resist Updat 2004;7:25-40.

24. Theys J, Jutten B, Dubois $L$, et al. The deletion mutant EGFRvIll significantly contributes to stress resistance typical for the tumour microenvironment. Radiother Oncol 
2009;92:399- 404.

25. Pelloski CE, Ballman KV, Furth AF, et al. Epidermal growth factor receptor variant III status defines clinically distinct subtypes of glioblastoma. J Clin Oncol 2007;25:22882294.

26. Shinojima N, Tada K, Shiraishi S, et al. Prognostic value of epidermal growth factor receptor in patients with glioblastoma multiforme. Cancer Res 2003;63:6962-6970.

27. Vosjan MJ, Perk LR, Roovers RC, et al. Facile labelling of an anti-epidermal growth factor receptor Nanobody with $68 \mathrm{Ga}$ via a novel bifunctional desferal chelate for immuno-PET. Eur J Nucl Med Mol Imaging 2011;38:753-763.

28. van Koningsbruggen $\mathrm{S}$, de Haard $\mathrm{H}$, de Kievit $\mathrm{P}$, et al. Llama-derived phage display antibodies in the dissection of the human disease oculopharyngeal muscular dystrophy. J Immunol Methods 2003;279:149-161.

29. Klingler-Hoffmann M, Bukczynska P Tiganis T. Inhibition of phosphatidylinositol 3-kinase signaling negates the growth advantage imparted by a mutant epidermal growth factor receptor on human glioblastoma cells. Int J Cancer 2003;105:331-339.

30. Narita Y, Nagane M, Mishima K, Huang HJ, Furnari FB Cavenee WK. Mutant epidermal growth factor receptor signaling down-regulates p27 through activation of the phosphatidylinositol 3-kinase/Akt pathway in glioblastomas. Cancer Res 2002;62:67646769.

31. Guo JY, Chen HY, Mathew R, et al. Activated Ras requires autophagy to maintain oxidative metabolism and tumorigenesis. Genes Dev 2011;25:460-470.

32. Kim MJ, Woo SJ, Yoon CH, et al. Involvement of autophagy in oncogenic K-Ras-induced malignant cell transformation. J Biol Chem 2011;286:12924-12932.

33. Lock R, Roy S, Kenific CM, et al. Autophagy facilitates glycolysis during Ras-mediated oncogenic transformation. Mol Biol Cell 2011;22:165-178.

34. Rouschop KM, van den Beucken $\mathrm{T}$, Dubois $\mathrm{L}$, et al. The unfolded protein response protects human tumor cells during hypoxia through regulation of the autophagy genes MAP1LC3B and ATG5. J Clin Invest 2010;120:127-141.

35. Niso-Santano M, Shen S, Adjemian S, et al. Direct interaction between STAT3 and EIF2AK2 controls fatty acid-induced autophagy. Autophagy 2013;9:415-417.

36. Shen S, Niso-Santano M, Adjemian S, et al. Cytoplasmic STAT3 represses autophagy by inhibiting PKR activity. Mol Cell 2012;48:667-680.

37. Wei Y, Zou Z, Becker N, et al. EGFR-Mediated Beclin 1 Phosphorylation in Autophagy Suppression, Tumor Progression, and Tumor Chemoresistance. Cell 2013;154:12691284.

38. Petiot A, Ogier-Denis E, Blommaart EF, Meijer AJ Codogno P. Distinct classes of phosphatidylinositol 3'-kinases are involved in signaling pathways that control macroautophagy in HT-29 cells. J Biol Chem 2000;275:992-998.

39. Funderburk SF, Wang QJ Yue Z. The Beclin 1-VPS34 complex--at the crossroads of 
autophagy and beyond. Trends Cell Biol 2010;20:355-362.

40. Selvakumaran M, Yao KS, Feldman MD O'Dwyer PJ. Antitumor effect of the angiogenesis inhibitor bevacizumab is dependent on susceptibility of tumors to hypoxiainduced apoptosis. Biochem Pharmacol 2008;75:627-638.

41. Choi BD, Archer GE, Mitchell DA, et al. EGFRvIll-targeted vaccination therapy of malignant glioma. Brain Pathol 2009;19:713-723.

42. Sampson JH, Heimberger AB, Archer GE, et al. Immunologic escape after prolonged progression-free survival with epidermal growth factor receptor variant III peptide vaccination in patients with newly diagnosed glioblastoma. J Clin Oncol 2010;28:47224729.

43. Sarkar S, Germeraad WT, Rouschop KM, et al. Hypoxia induced impairment of NK cell cytotoxicity against multiple myeloma can be overcome by IL-2 activation of the NK cells. PLoS One 2013;8:e64835.

44. Noman MZ, Janji B, Kaminska B, et al. Blocking hypoxia-induced autophagy in tumors restores cytotoxic T-cell activity and promotes regression. Cancer Res 2011;71:59765986.

45. Li G, Mitra SS, Monje M, et al. Expression of epidermal growth factor variant III (EGFRvIII) in pediatric diffuse intrinsic pontine gliomas. J Neurooncol 2012;108:395-402.

46. Liu XJ, Wu WT, Wu WH, et al. A minority subpopulation of CD133(+)/EGFRvIII(+) /EGFR(-) cells acquires stemness and contributes to gefitinib resistance. CNS Neurosci Ther 2013;19:494-502. 
$\longrightarrow$ 
Nederlandse samenvatting 


\section{Samenvatting}

Deze thesis beschrijft hoe de Epidermale Groei Factor Receptor (EGFR) en een van de meest voorkomende afwijkende (mutant) vorm, variant III (EGFRvIII), bijdragen aan de agressiviteit van een tumor. Tevens worden nieuwe behandeling mogelijkheden besproken voor patiënten met een tumor met ontregelde EGFR signalering.

EGFR is een lid van de ErbB familie van receptoren welke bestaat uit 4 leden; EGFR (Erb1, HER1), Erb2 (HER2), Erb3 (HER3) en Erb4 (HER4). EGFR wordt geactiveerd door binding van een ligand, hierdoor veranderd de receptor van conformatie. Door deze vormverandering kunnen twee EGFR moleculen een interactie aangaan, dimeriseren (homo-dimeren) of dimeriseren met een ander lid uit de ErbB-familie (hetero-dimeren). Dimerisatie leidt tot phosphorylatie aan de binnenkant van de cel waardoor signalering geactiveerd wordt. EGFR werkt via verschillende signaleringroutes die de cel aanzetten tot gen expressie, cytoskelet veranderingen, remming van geprogrammeerde celdood (apoptose), toename van celgroei en deling en zorgt ervoor dat de tumorcel een fenotype krijgt dat lijkt op dat van 'tumorstamcellen'. Niet lang na de ontdekking van de ErbB receptor familie, in de vroege jaren '80, werden deze al geassocieerd met kanker. In veel kankersoorten zijn de ErbB signalering routes hyper-geactiveerd door o.a. overproductie van liganden, overproductie van receptoren (amplificatie) of door continue activatie van de receptoren.

Hierdoor wordt amplificatie van het EGFR gen geassocieerd met resistentie tegen radiotherapie door de activatie van overlevingssignalering en DNAherstel mechanismen door EGFR. Onderbreking van EGFR signalering kan daarom beschouwd worden als een mogelijke strategie om tumoren gevoeliger te maken voor behandeling. Ondanks het feit dat er al veel onderzoek is gedaan naar het remmen van EGFR signalering met behulp van medicijnen die de phosphorylatie tegen gaan (TKIs) en met antilichamen die de dimerisatie voorkomen, blijken deze in de praktijk slechts effectief te zijn in een beperkte groep patiënten.

Recent onderzoek heeft uitgewezen dat kanker gerelateerde mutaties in bijvoorbeeld het k-RAS- of PTEN gen kan leiden tot ongecontroleerde celgroei en deling ondanks de blokkade van EGFR signalering. Verder kunnen 
verschillende strategieën om EGFR signalering te blokkeren (TKIs versus antilichamen) verschillende resultaten kunnen opleveren in dezelfde tumor. Compensatie via andere receptoren, inclusief mutaties in EGFR, zijn waarschijnlijk de reden hiervoor.

De meest voorkomende mutatie van EGFR is de zogenoemde variant 3 of EGFRvIII. EGFRvIII staat bekend voor zijn bedrage aan de radioresistentie van tumorcellen, o.a. door zijn bedrage aan DNA herstel na bestraling. Verder leidt EGFRvIll expressie tot resistentie tegen Gefitinib (een TKI) en tot constante EGFR signalering. We hebben daarom onderzocht of tumorcellen met EGFRvIII expressie behandeld kunnen worden met Cetuximab, een antilichaam tegen EGFR. Cetuximab voorkomt de binding van een ligand aan domein I en III van EGFR. EGFRvIII mist een groot deel van domein I en II. Daarom stelde wij ons de vraag of Cetuximab zou binden aan EGFRvIll en of deze binding dan een functionele therapeutische waarde zou hebben? In hoofdstuk 2 laten wij zien dat Cetuximab inderdaad kan binden aan EGFRvIII. Deze binding leidde tot een verminderde expressie van EGFRvIII op de cel. Dit had echter geen effect op de groeisnelheid van deze cellen. Mogelijk geeft de expressie van EGFRvIII onder normale omstandigheden geen groeivoordeel of de vermindering in EGFRvIll expressie was niet voldoen om een effect te hebben. Verder leidde de behandeling met Cetuximab ook niet tot radiosensitisatie, suggererend dat voldoende EGFRvIII nog aanwezig is om radioresistentie te handhaven. Toch was de ERK signalering verminderd in EGFRvIII cellen wanneer deze behandeld werden met Cetuximab. Dit suggereert dat Cetuximab EGFRvIII signalering vermindert en zou mogelijk gebruikt kunnen worden voor de behandeling van tumoren met lage EGFRvIII expressie.

In de dagelijkse klinische routine worden de hoeveelheid van en de mutaties in EGFR bepaald om een optimale behandeling van de patiënt te bereiken. Tevens worden mutaties in signaleringmoleculen van EGFR bepaald omdat mutaties hierin kunnen leiden tot ongevoeligheid van de tumor tegen anti-EGFR behandelingen. De expressie van EGFRvIII wordt echter niet meegenomen in deze analyse ondanks bewezen klinische relevantie en de verlaagde effectiviteit van de anti-EGFR therapie door EGFRvIII. Tumorcellen die EGFRvIII tot expressie brengen zijn resistenter tegen Gefitinib en hebben dus 
meer en hogere dosissen nodig. Deze hogere dosissen zijn echter niet voldoende om alle processen die leiden tot therapieresistentie van EGFRvIII stil te leggen. Mogelijk is het ontbreken van een goed commercieel verkrijgbaar antilichaam specifiek voor EGFRvIII een reden voor het niet testen op EGFRvIII expressie. Wij hebben daarom getracht een EGFRvIII specifiek antilichaam te genereren afgeleid van een lama antilichaam (hoofdstuk 3). Dit EGFRvIII specifiek mini-antilichaam of nanobody zou gebruikt kunnen worden in combinatie met een eerder gevonden nanobody tegen normaal EGFR, om de expressie niveaus van EGFR en EGFRvIll te bepalen. Met deze nanobodies kan dit zowel in de patiënt als in verwijderd tumormateriaal. Het ontwikkelde antilichaam had hoge affiniteit voor EGFRvIII, maar bleek ook te binden aan normaal EGFR wanneer dit in grote hoeveelheden aanwezig was. Dit maakt het nanobody tegen EGFRvIII ongeschikt voor het stellen van een goede diagnose omdat EGFRvIII expressie vaak gepaard gaat met een hoge expressie van normaal EGFR.

In hoofdstuk 4 hebben we onderzocht hoe tumorcellen met EGFR en EGFRvIII expressie verschillend omgaan met de stress die hoort bij het tumor-micro milieu, zoals lage zuurstof concentratie of weinig voedingsstoffen. Wij zagen dat cellen met EGFRvIII sneller bleven groeien wanneer ze weinig zuurstof of voedingsstoffen kregen. Dit bleek specifiek voor EGFRvIII aangezien cellen met EGFR dit niet vertoonden. Tevens observeerden we dat de cellen met EGFRvIII beter overleefden dan controle cellen of cellen met EGFR wanneer ze weinig zuurstof of voedingsstoffen kregen. Ook tumoren die voortkwamen uit de cellen met EGFRvIll groeiden sneller. Deze verhoogde groeisnelheid kon worden teruggebracht wanneer EGFRvIll productie in de cel werd uitgezet. Deze bevindingen suggereren dat het remmen van EGFRvIII expressie en/of een blokkade in de signaleringsroute een veelbelovende strategie kunnen zijn in de behandeling van tumoren met EGFRvIll expressie.

Cellen met EGFRvIII groeien sneller en overleven beter wanneer ze blootgesteld worden aan weinig zuurstof (hypoxie) of voedingsstoffen (starvatie) vergeleken met cellen zonder EGFRvill. In hoofdstuk 5 hebben we aangetoond dat remming van autofagie (een proces dat de cel gebruikt om beschadigde onderdelen af te breken en hergebruiken) in cellen met EGFRvIII leidt tot een verlaagde groei en overleving wanneer ze blootgesteld worden aan hypoxie of 
starvatie. Cellen met EGFRvIll zijn zelfs gevoeliger voor remming van autofagie wanneer ze blootgesteld worden aan hypoxie of starvatie dan cellen zonder EGFRvIII. Vergelijkbaar met eerdere experimenten, zagen we dat tumoren met EGFRvIII expressie sneller groeiden en ook sneller terug groeiden na radiotherapie in vergelijk met tumoren zonder EGFRvIII expressie. Opvallend was dat chloroquine ( $C Q$, een medicijn dat autofagie remt) behandeling deze versnelde groei en hergroei teniet kon doen. De onderliggende mechanismen hierbij moeten nog worden uitgezocht. Intuïtief zou men verlaagde autofagie verwachten in cellen met EGFRvIll omdat een van de belangrijkste signaleringsroutes van EGFR, de zo gehete mTOR signalering, juist lijdt tot verlaagde autofagie. Voorlopige data wijzen erop dat een ander signaleringsroute, de RAS-RAF signalering, een meer belangrijke rol in de verhoogde autofagie van cellen met EGFRvIll speelt.

Autofagie wordt noch transcriptioneel noch translationeel gereguleerd. Desondanks is voldoende transcriptie en translatie nodig om voldoende autofagie flux te behouden. Signal Transducer and Activator of Transcription 3 (STAT3) signalering zou verantwoordelijk kunnen zijn voor het behoud van de verhoogde autofagie flux in EGFRvIll cellen tijdens zuurstof tekort via Protein Kinase $R(P K R)$ en elF2 $\alpha$. Recent is een vierde autofagie regulerende signalering van EGFR ontdekt. EGF geactiveerd EGFR phosphoryleerd Beclin 1 op 3 tyrosines waardoor Beclin 1 dimeren vormt en niet meer aan het Vps34 complex kan binden en daardoor autofagie niet kan activeren. Dit kan mogelijk verklaren waarom cellen met EGFR overexpressie lage base autofagie flux hebben zoals beschreven in hoofdstuk 6 . Echter, de exacte mechanismen die hierbij een rol spelen moeten nog verder worden uitgezocht.

Analyse van een cohort van 52 glioblastoma patiënten (18 conventioneel en 34 met additioneel $C Q$ behandeld) werden grote toenames in overleving gezien. Patiënten met een EGFRvIll negatieve tumor hadden een toename in overleving van 5 naar 10 maanden na diagnose, waar patiënten met een EGFRvIll positieve tumor een toename van 3 naar 15 maanden hadden. Deze resultaten zijn in lijn met de verkregen preklinische data. Enige voorzichtigheid is echter geboden omdat het een relatief kleine groep is. Echter, deze veel belovende resultaten dienen verder onderzocht te worden in gerandomiseerde studies met patiënten stratificatie gebaseerd op EGFRvIII expressie. 
Samengevat, de inhibitie van autofagie door middel van $C Q$ is een veelbelovende toevoeging voor de therapie van EGFRvIll positieve tumoren. Om de relatie tussen niet-gemuteerd EGFR en autofagie te bepalen werden glioblastoma cellen met EGFR overexpressie in een xenograft model gebruikt en de effectiviteit van $C Q$ behandeling getest, zoals beschreven in hoofdstuk 6. $C Q$ behandeling leidde tot een afname in de groei van tumoren met EGFR overexpressie. Zoals verwacht waren tumoren met EGFR overexpressie zeer resistent tegen radiotherapie. Deze resistente tumoren konden gevoelig gemaakt worden voor radiotherapie door $C Q$ behandeling, zoals gezien met de EGFRvIII tumoren (hoofdstuk5). CQ behandeling op zich zelf vertraagde de groei van tumoren met EGFR overexpressie zelfs meer dan de radiotherapie (enkele dosis van 10Gy).

Opvallend was dat dit niet kwam door de afname van de hypoxische fractie, zoals we in het verleden gezien hebben. Noch de necrotische of de apoptotische fractie leken beïnvloed te worden door $C Q$ behandeling. Dit suggereert dat niet alleen de cellen met weinig zuurstof maar ook andere cellen getarget worden en geeft aan dat cellen met EGFR overexpressie sterk afhankelijk zijn van autofagie voor hun overleving. Tevens werd in xenografts van primaire hoofd-hals tumoren een positieve relatie gevonden tussen LC3b en EGFR RNA en eiwit expressie. Dit suggereert dat EGFR betrokken is in de productie van LC3b. Ondanks dat LC3b voornamelijk gemaakt wordt in hypoxische gebieden van een tumor, werd deze correlatie niet gevonden in tumoren met EGFR overexpressie. Dit suggereert dat andere cellen dan die met te weinig zuurstof (bij-voorbeeld die cellen die te weinig voedingsstoffen krijgen) getarget worden. Dit vermoeden werd versterkt doordat de meeste LC3b expressie in hoofd-hals tumoren met EGFR overexpressie gevonden werd in de niet-hypoxische gebieden. Samengevat, de tumoren met hoge EGFR expressie gebruikt in deze studie zijn erg afhankelijk van autofagie voor hun groei, overleving en resistentie tegen radiotherapie. Het blokkeren van autofagie kan daarom een goede additionele behandeling zijn voor tumoren met EGFR overexpressie.

\section{Tot slot}

Sinds de ontdekking in begin jaren 80 is er veel onderzoek gedaan naar EGFR. 
In veel verschillende kankersoorten is de EGFR signaleringsroute over actief om verschillende redenen zoals overproductie van de liganden, overproductie van de receptor of constante activatie van de receptor door mutaties. Daarom worden tumoren in klinische setting getest op EGFR en gerelateerde signalering moleculen om de juiste diagnose en behandeling te bepalen. Opvallend hierbij is dat EGFRvIll hierin niet wordt meegenomen ondanks een toenemende hoeveelheid aan onderzoeks- en klinische data die suggereren dat EGFRvIll expressie een negatieve invloed heeft op de behandeling en de overleving van de patiënt. Daarom zou in een klinische setting het routinematig testen van tumoren op EGFRvIll overwogen moeten worden.

Veel therapieën gericht tegen EGFR zoals TKIs en antilichamen die zeer effectief zijn in het laboratorium blijken maar minimaal succesvol in de klinische praktijk. Tumoren kunnen resistent worden tegen de behandeling door compensatie mechanismen te activeren of de expressie van EGFRvIII.

In dit proefschrift beschrijven we een potentieel nieuwe toevoeging aan de therapie om tumoren met EGFR en EGFRvIII overexpressie te behandelen. We hebben laten zien dat tumoren met zowel EGFR als EGFRvIII overexpressie zeer sterk reageren op inhibitie van autofagie door middel van $C Q$. Verder blijkt dat $C Q$ in combinatie met conventionele behandeling goed werkt en zelfs beter in patiënten met een tumor met EGFRvIII. Meer en grotere klinische trials zijn echter nodig om de effectiviteit van $C Q$ in de behandeling van (EGFRvIII positieve) hersentumoren vast te stellen.

Het ontrafelen van de exacte autofagie signalering routes van EGFR en EGFRvIII in tumoren zou nieuwe inzichten kunnen opleveren over hoe deze meer efficiënt behandeld kunnen worden. Veel inhibitoren van signalering moleculen van EGFR zoals PI3K, mTOR, RAS of RAF zijn momenteel beschikbaar voor onderzoek of worden zelfs al toegepast in de kliniek. De combinatie van deze inhibitoren en anti-EGFR therapie zou nieuwe behandeling strategieën kunnen opleveren.

Men beseft meer en meer dat enkelvoudige therapieën niet effectief genoeg zijn tegen de meeste kanker soorten en dat nieuwe behandelingsstrategieën gezocht moeten worden in combinaties van bestaande of nieuwe therapieën. In dat licht zouden patiënten met een glioblastoma behandeld kunnen worden met Avastin (Bevacizumab, een antilichaam tegen Vascular Endothelial growth 
factor (VEGF) welk de aanmaak van nieuwe bloedvaten tegen gaat) in combinatie met inhibitie van autofagie en radiotherapie. Behandeling met Bevacizumab leidt tot een toename van de hypoxische fractie van een tumor. Wanneer dit dan gecombineerd wordt met $C Q$ is een toename in tumorceldood te verwachten en wordt de effectiviteit van Bevacizumab als enkelvoudige therapie verhoogd. Hiermee kan de hergroei van de tumor na bestraling uitgesteld of zelfs voorkomen worden.

Een andere mogelijkheid om tumoren met EGFRvIII expressie te bestrijden is door middel van vaccinatie. EGFRvIll is ideaal als vaccinatie kandidaat omdat het alleen voorkomt op tumorcellen en niet op normaal weefsel. Vaccinatie van patiënten met een EGFRvIII positieve tumor met een EGFRvIII peptide leverde een 5-voudige toename in overleving. Echter, Natural Killer (NK) cellen zijn minder effectief in een hypoxische omgeving en tumor cellen gebruiken autofagie als bescherming tegen cytotoxische $T$ lymfocyten (CTLS). De combinatie van radiotherapie, $C Q$ en vaccinatie zou daarom alle normoxische en hypoxische cellen moeten vernietigen. Deze benadering richt zich niet alleen op 'normale' tumor cellen maar ook op de zeer radioresistente glioblastoma kanker stem cellen (CSC) welke EGFRvIII tot expressie brengen.

Samengevat, ondanks enkele decennia aan onderzoek, nieuwe anti-EGFR medicijnen en klinische trials, blijven tumoren met EGFR en EGFRvIII expressie zeer moeilijk te behandelen. Daarom is meer onderzoek nodig om alle mechanismen die hierbij een rol spelen te onderzoeken een zo best mogelijke behandeling te bereiken. 
Dankwoord 


\section{Dankwoord}

\section{Bedankt !!}

Heel veel dank aan iedereen die een bijdrage (in welke vorm dan ook) heeft geleverd aan het tot stand komen van dit proefschrift.

Allereerst wil ik mijn promotor bedanken. Philippe, bedankt voor het vertrouwen dat je in mij had toen ik met het verzoek kwam om te mogen promoveren binnen jouw afdeling. Door je ontzettend drukke functie hebben helaas we weinig overleg gehad tijdens mijn promotietraject. Desondanks wist je toch altijd precies waar ik mee bezig was en had je altijd wel een of meerdere suggesties om mijn onderzoek te verbeteren of uit te breiden.

Natuurlijk veel dank aan de rest van mijn promotie team, Guido Lammering, Jan Theys en Kasper Rouschop, voor jullie tijd, begeleiding en steun de afgelopen jaren.

Ook vele malen dank aan alle medewerkers van Maastro lab en Maastro clinic, de afdeling pathologie van het azM, afdeling pathologie van het atrium medisch centrum Heerlen, afdeling moleculaire cel biologie van de universiteit Maastricht, de afdeling celbiologie van de universiteit Utrecht en afdeling radiotherapie van het Radboudumc Nijmegen. Zonder jullie had ik dat boekje niet kunnen maken.

Alle leden van mijn lees- en beoordelingscommissie, prof.dr. Ramaekers, prof.dr. de Bruïne, prof.dr. Rodemann, dr. Bussink, prof.dr. Speel, dr. Langen en dr. Dullens, dank voor jullie interesse voor mijn onderzoek en de tijd die jullie hebben genomen om mijn proefschrift te lezen. 
Curriculum Vitae 


\section{Curriculum Vitae}

Barry Jutten was born on September 21st in Stein, the Netherlands. After high school at the Groenewald in Stein, he studied chemistry at the University of Utrecht for two years before he switched to the University of Applied Sciences (Hogeschool Limburg) to study biochemistry, from which he graduated in 1997. In the period from 1997 to 2006 Barry worked as a research technician at the department of Pathology at Maastricht University, and from 2006 to 2008 he worked as a research technician at the department of Radiotherapy (MAASTRO Lab) at Maastricht University. In 2008 Barry started his Master in Molecular Life Sciences at Maastricht University and started as a PhD student within MAASTRO Lab simultaneously. He studied how EGFR and EGFRvIII contribute to a more aggressive tumour phenotype and how the inhibition of autophagy could contribute to the treatment of tumours with deregulated EGFR signalling under supervision of Prof. dr. Ph. Lambin. He finished his PhD thesis in 2014 and continued this research as a post-doc at MAASTRO. 
List of publications 


\section{List of publications}

Jutten B, Rouschop KM.

EGFR signaling and autophagy dependence for growth, survival, and therapy resistance.

Cell Cycle 2014 Jan;13(1):42-51

Barry Jutten, Tom G.H. Keulers, Marco B.E. Schaaf, Kim G.M. Savelkouls, Jan Theys, Paul N. Span, Marc A. Vooijs, Johan Bussink, Kasper M.A.

Rouschop

EGFR overexpressing cells and tumors are dependent on autophagy for growth and survival.

Radiother Oncol. 2013 Sep;108(3):479-83

Marco BE Schaaf, Dan Cojocari, Tom GH Keulers, Barry Jutten, Maud Starmans, Monique D Jong, Adrian Begg, Kim GM Savelkouls, Johan Bussink, Marc Vooijs, Bradly G Wouters, Kasper MA Rouschop The autophagy associated gene, ULK1, promotes tolerance to chronic and acute hypoxia.

Radiother Oncol. 2013 Sep;108(3):529-34

van den Beucken T, Magagnin MG, Jutten B, Seigneuric R, Lambin P, Koritzinsky M, Wouters BG.

Translational control is a major contributor to hypoxia induced gene expression.

Radiother Oncol. 2011 Jun;99(3):379-84.

Theys J, Jutten B, Habets R, Paesmans K, Groot AJ, Lambin P, Wouters BG, Lammering G, Vooijs M.

E-Cadherin loss associated with EMT promotes radioresistance in human tumor cells.

Radiother Oncol. 2011 Jun;99(3):392-7 
Barry Jutten, Ludwig Dubois, Younan Li, Hugo Aerts, Bradly G Wouters, Philippe Lambin, Jan Theys, Guido Lammering

Binding of cetuximab to the EGFRvIll deletion mutant and its biological consequences in malignant glioma cells. Radiother Oncol. 2009 Sep;92(3):393-8.

Jan Theys, Barry Jutten, Ludwig Dubois, Kasper Rouschop, Roland K Chiu, Younan Li, Kim Paesmans, Philippe Lambin, Guido Lammering, Bradly G Wouters

The deletion mutant EGFRvIII significantly contributes to stress resistance typical for the tumour microenvironment.

Radiother Oncol. 2009 Sep;92(3):399-404.

Twan van den Beucken, Marianne Koritzinsky, Hanneke Niessen, Ludwig Dubois, Kim Savelkouls, Hilda Mujcic, Barry Jutten, Juraj Kopacek, Sylvia Pastorekova, Albert J. van der Kogel, Philippe Lambin, Willem Voncken, Kasper M.A. Rouschop and Bradly G. Wouters Hypoxia-induced expression of carbonic anhydrase 9 is dependent on the unfolded protein response.

JBC. 2009 Sep 4;284(36):24204-12.

Sluimer J, Gasc J, Hamming I, van Goor H, Michaud A, van den Akker L, Jütten B, Cleutjens J, Bijnens A, Corvol P, Daemen M, Heeneman S. Angiotensin-converting enzyme 2 (ACE2) expression and activity in human carotid atherosclerotic lesions.

J Pathol. 2008 Jul;215(3):273-9.

Weppler SA, Li Y, Dubois L, Lieuwes N, Jutten B, Lambin P, Wouters BG, Lammering $\mathrm{G}$.

Expression of EGFR variant vIll promotes both radiation resistance and hypoxia tolerance.

Radiother Oncol. 2007 Jun;83(3):333-9. 
Aerts HJ, Dubois L, Hackeng TM, Straathof R, Chiu RK, Lieuwes NG, Jutten B, Weppler SA, Lammering G, Wouters BG, Lambin P.

Development and evaluation of a cetuximab-based imaging probe to target EGFR and EGFRvIII.

Radiother Oncol. 2007 Jun;83(3):326-32.

Magagnin MG, Sergeant K, van den Beucken T, Rouschop KM, Jutten B, Seigneuric R, Lambin P, Devreese B, Koritzinsky M, Wouters BG.

Proteomic analysis of gene expression following hypoxia and reoxygenation reveals proteins involved in the recovery from endoplasmic reticulum and oxidative stress.

Radiother Oncol. 2007 Jun;83(3):340-5.

Bijnens AP, Gils A, Jutten B, Faber BC, Heeneman S, Kitslaar PJ, Tordoir $\mathrm{JH}$, de Vries CJ, Kroon AA, Daemen MJ, Cleutjens KB.

Vasculin, a novel vascular protein differentially expressed in human atherogenesis.

Blood 2003 Oct 15;102(8):2803-10.

G.L. Scheffer, A.W. Reurs, B. Jutten, S.H.W. Beiboer, R. van Amerongen, M. Schoester, E.A.C. Wiemer, H.R. Hoogenboom, R.J. Scheper

Selection and characterization of a phage-displayed human antibody (Fab) reactive to the lung resistance-related major vault protein.

Br J Cancer. 2002 Mar 18;86(6):954-62. 